JOURNAL OF THE

AMERICAN MATHEMATICAL SOCIETY

Volume 22, Number 3, July 2009, Pages 739-769

S 0894-0347(08)00603-6

Article electronically published on March 31, 2008

\title{
STUDY OF A Z-FORM OF THE COORDINATE RING OF A REDUCTIVE GROUP
}

\author{
G. LUSZTIG
}

\section{INTRODUCTION}

In his famous paper C1. Chevalley associated to any root datum of adjoint type and to any field $k$ a certain group (now known as a Chevalley group) which in the case where $k=\mathbf{C}$ was the usual adjoint Lie group over $\mathbf{C}$ and which in the case where $k$ is finite led to some new families of finite simple groups. Let $\mathbf{O}^{\prime}$ be the coordinate ring of a connected semisimple group $G$ over $\mathbf{C}$ attached to a fixed (semisimple) root datum. In a sequel C2 to C1, Chevalley defined a Zform of $\mathbf{O}^{\prime}$. Later, another construction of such a $\mathbf{Z}$-form was proposed by Kostant [Ko. Kostant notes that $\mathbf{O}^{\prime}$ can be viewed as a "restricted" dual of the universal enveloping algebra $\mathbf{U}$ of Lie $G$; he defines a $\mathbf{Z}$-form $\mathbf{U}_{\mathbf{Z}}$ of $\mathbf{U}$ ("the Kostant $\mathbf{Z}$ form") and then defines the $\mathbf{Z}$-form $\mathbf{O}_{\mathbf{Z}}^{\prime}$ as the set of all elements in $\mathbf{O}^{\prime}$ which take integral values on $\mathbf{U}_{\mathbf{Z}}$. Then for any commutative ring $A$ with 1 he defines $\mathbf{O}_{A}^{\prime}$ as $A \otimes \mathbf{O}_{\mathrm{Z}}^{\prime}$; this is naturally a Hopf algebra over $A$. It follows that the set $G_{A}$ of $A$-algebra homomorphisms $\mathbf{O}_{A}^{\prime} \rightarrow A$ has a natural group structure. Thus the root datum gives rise to a family of groups $G_{A}$, one for each $A$ as above.

Unlike Chevalley's approach which was based on a choice of a faithful representation of $G$, Kostant's approach is direct (no choices involved) and generalizes to the quantum case.

In this paper we develop the theory of Chevalley groups following Kostant's approach. We shall prove that:

(I) If $A$ is an algebraically closed field, then $\mathbf{O}_{A}^{\prime}$ is the coordinate algebra of a connected semisimple algebraic group over $A$ corresponding to the given root datum. (We treat the reductive case at the same time.) Note that (I) was stated without proof in $[\mathrm{Ko}$.

In this paper we note that Kostant's definition can be reformulated by replacing U by a "modified enveloping algebra". The theory is then developed using extensively the theory of canonical bases of such modified enveloping algebras (presented in [L1]), coming from quantum groups. (See the Notes in [L1] for references to original sources concerning canonical bases.)

We now present the content of this paper in more detail.

Let $A$ be a fixed commutative ring with 1 with a given invertible element $v \in A$.

Received by the editors September 19, 2007.

2000 Mathematics Subject Classification. Primary 20G99.

The author was supported in part by the National Science Foundation.

(C)2008 American Mathematical Society Reverts to public domain 28 years from publication 
In $\S 1$ we recall the definition and some properties of the modified (quantized) enveloping algebra $\dot{\mathbf{U}}_{A}$ over $A$ and its canonical basis $\dot{\mathbf{B}}$. We also define a "completion" $\hat{\mathbf{U}}_{A}$ of $\dot{\mathbf{U}}_{A}$ which consists of formal (possibly infinite) $A$-linear combinations of elements in $\dot{\mathbf{B}}$. We show that the multiplication and "comultiplication" of $\dot{\mathbf{U}}_{A}$ extend naturally to $\hat{\mathbf{U}}_{A}$.

In $\S 2$ we introduce some invertible elements $s_{i, e}^{\prime}$ of $\hat{\mathbf{U}}_{A}$ (where $i$ corresponds to a simple reflection and $e= \pm 1$ ). We show that conjugation by $s_{i, e}^{\prime}$ restricted to $\dot{\mathbf{U}}_{A}$ is essentially the action of a generator in the braid group action on $\dot{\mathbf{U}}_{A}$ studied in L1. Thus $s_{i, e}^{\prime}$ plays the same role as an element considered in a similar context (with $A=\mathbf{C}(v)$ ) by Soibelman [So]. But while Soibelman's element is not explicit and its integrality properties are not clear, our element $s_{i, e}^{\prime}$ is remarkably simple and has obvious integrality properties.

In $\S 3$ we define following [L1, 29.5.2] the Hopf algebra $\mathbf{O}_{A}$ (a quantum analogue of $\mathbf{O}_{A}^{\prime}$ above). We prove that the $A$-algebra $\mathbf{O}_{A}$ is finitely generated (see Proposition $3.3)$ with an explicit set of generators. In 3.7 we show that the $A$-algebra $\mathbf{O}_{A}$ can be imbedded into the tensor product of two simpler algebras. (For a closely related result in the case where $A=\mathbf{Q}(v)$, see [Jo, 9.3.13].) In the case where $v=1$ in $A$ these simpler algebras can be explicitly described in terms of the braid group action; see 3.13. We deduce that, if $A$ is an integral domain and $v=1$ in $A$, then $\mathbf{O}_{A}$ is an integral domain; see Theorem 3.15. (For a similar result in the case where $A=\mathbf{Q}(v)$, see [Jo, 9.1.9].)

In $\S 4$ we assume that $v=1$ in $A$ and we introduce the group $G_{A}$ in analogy with Ko. In Theorem 4.11 we show that $\mathbf{O}_{A}$ has a property like (I) above.

In $\S 5$ we identify (assuming that $v=1$ in $A$ ) our $\mathbf{O}_{A}$ with Kostant's $\mathbf{O}_{A}^{\prime}$.

We refer the reader to Borel's paper in B2] and to Steinberg's book St for expositions of Chevalley's approach to his groups which make use of the Kostant $\mathbf{Z}$-form $\mathbf{U}_{\mathbf{Z}}$. We also refer the reader to Demazure's paper in [DG, Exposé XXV] where another proof of the existence of a reductive group over $\mathbf{Z}$ with a given root datum is given, assuming that the corresponding group over $\mathbf{C}$ is already known.

Notation. If $A^{\prime}$ is a commutative ring with 1 and $S$ is a set, we denote by $A^{\prime}[S]$ the free $A^{\prime}$-module with basis indexed by $S$.

\section{Contents}

1. The algebras $\dot{\mathbf{U}}_{A}, \hat{\mathbf{U}}_{A}$

2. The elements $s_{i, e}^{\prime}, s_{i, e}^{\prime \prime}$ of $\hat{\mathbf{U}}_{A}$

3. The Hopf algebra $\mathbf{O}_{A}$

4. The group $G_{A}$

5. From enveloping algebras to modified enveloping algebras

\section{The algebras $\dot{\mathbf{U}}_{A}, \widehat{\mathbf{U}}_{A}$}

1.1. In this section we recall the definition of the modified quantized enveloping algebra $\dot{\mathbf{U}}_{A}$ (over $A$ ) attached to a root datum and we recall the definition and some of the properties of the canonical basis $\dot{\mathbf{B}}$ of $\dot{\mathbf{U}}_{A}$. We also study a certain completion $\hat{\mathbf{U}}_{A}$ of $\dot{\mathbf{U}}_{A}$.

We fix a root datum as in [L1, 2.2]. This consists of two free abelian groups of finite type $Y, X$ with a given perfect pairing $\langle\rangle:, Y \times X \rightarrow \mathbf{Z}$ and a finite set $I$ with given imbeddings $I \rightarrow Y \quad(i \mapsto i)$ and $I \rightarrow X \quad\left(i \mapsto i^{\prime}\right)$ such that $\left\langle i, i^{\prime}\right\rangle=2$ for 
all $i \in I$ and $\left\langle i, j^{\prime}\right\rangle \in-\mathbf{N}$ for all $i \neq j$ in $I$; in addition, we are given a symmetric bilinear form $\mathbf{Z}[I] \times \mathbf{Z}[I] \rightarrow \mathbf{Z}, \nu, \nu^{\prime} \mapsto \nu \cdot \nu^{\prime}$ such that $i \cdot i \in 2 \mathbf{Z}_{>0}$ for all $i \in I$ and $\left\langle i, j^{\prime}\right\rangle=2 i \cdot j / i \cdot i$ for all $i \neq j$ in $I$. We assume that the matrix $(i \cdot j)_{i, j \in I}$ is positive definite.

Let $X^{+}=\{\lambda \in X ;\langle i, \lambda\rangle \geq 0$ for all $i \in I\}$. For $\lambda, \lambda^{\prime}$ in $X$ we write $\lambda \geq \lambda^{\prime}$ or $\lambda^{\prime} \leq \lambda$ if $\lambda-\lambda^{\prime} \in \sum_{i \in I} \mathbf{N} i^{\prime}$. The image of $\nu \in \mathbf{Z}[I]$ under the homomorphism $\mathbf{Z}[I] \rightarrow X$ such that $i \mapsto i^{\prime}$ for $i \in I$ is denoted again by $\nu$.

Let $W$ be the (finite) subgroup of $\operatorname{Aut}(Y)$ generated by the involutions $s_{i}$ : $y \mapsto y-\left\langle y, i^{\prime}\right\rangle i \quad(i \in I)$ or equivalently the subgroup of $\operatorname{Aut}(X)$ generated by the involutions $s_{i}: x-\langle i, x\rangle i^{\prime} \quad(i \in I)$; these two subgroups may be identified by taking contragredients. For $i \neq j$ in $I$ let $n_{i j}=n_{j i}$ be the order of $s_{i} s_{j}$ in $W$. Let $l: W \rightarrow \mathbf{N}$ be the standard length function on $W$ with respect to $\left\{s_{i} ; i \in I\right\}$. Let $w_{0} \in W$ be the unique element such that $l\left(w_{0}\right)$ is maximal.

1.2. Let $v$ be an indeterminate. Let $\mathcal{A}=\mathbf{Z}\left[v, v^{-1}\right]$. For $i \in I$ we set $v_{i}=v^{i \cdot i / 2}$.

We fix a commutative ring $A$ with 1 with a given ring homomorphism $\mathcal{A} \rightarrow A$ respecting 1. For $\alpha \in \mathcal{A}$ we shall often denote the image of $\alpha$ under $\mathcal{A} \rightarrow A$ again by $\alpha$. Whenever we write $A=\mathbf{Q}(v)$ or $A=\mathcal{A}$, we shall understand that $A$ is regarded as an $\mathcal{A}$-algebra in the obvious way. Whenever we write $A=\mathbf{Q}$ or $A=\mathbf{Z}$, we shall understand that $A$ is regarded as an $\mathcal{A}$-algebra with $v=1$ in $A$.

Let $A^{\circ}$ be the group of invertible elements of the ring $A$.

An $A$-linear map $\phi: H \rightarrow H^{\prime}$ where $H, H^{\prime}$ are $A$-modules is said to be a split injection if there exists an $A$-linear map $\psi: H^{\prime} \rightarrow H$ such that $\psi \phi=1$.

1.3. Let $\mathbf{f}$ be the associative $\mathbf{Q}(v)$-algebra with 1 defined as in [L1, 1.2.5] or equivalently by the generators $\theta_{i}(i \in I)$ and the quantum Serre relations

$$
\sum_{p, p^{\prime} \in \mathbf{N} ; p+p^{\prime}=1-\left\langle i, j^{\prime}\right\rangle}(-1)^{p^{\prime}}\left(\theta_{i}^{p} /[p]_{i}^{!}\right) \theta_{j}\left(\theta_{i}^{p^{\prime}} /\left[p^{\prime}\right]_{i}^{!}\right)
$$

for $i \neq j$ in $I$, where $[p]_{i}^{!}=\prod_{s=1}^{p}\left(v_{i}^{s}-v_{i}^{-s}\right) /\left(v_{i}-v_{i}^{-1}\right)$ for $p \in \mathbf{N}$. We have a direct sum decomposition $\mathbf{f}=\bigoplus_{\nu \in \mathbf{Z}[I]} \mathbf{f}_{\nu}$ as a vector space, where $\mathbf{f}_{\nu}$ is spanned by words in $\theta_{i}$ in which the number of apparitions of $\theta_{i}$ is the coefficient of $i$ in $\nu$, for all $i \in I$. For $i \in I, n \in \mathbf{Z}$ we set $\theta_{i}^{(n)}=\theta_{i}^{n} /[n !]_{i} \in \mathbf{f}$ if $n \geq 0$ and $\theta_{i}^{(n)}=0$ if $n<0$. Let $\mathbf{f}_{\mathcal{A}}$ be the $\mathcal{A}$-subalgebra with 1 of $\mathbf{f}$ generated by the elements $\theta_{i}^{(n)}$ with $i \in I, n \in \mathbf{Z}$. We have $\mathbf{f}_{\mathcal{A}}=\bigoplus_{\nu \in \mathbf{Z}[I]} \mathbf{f}_{\nu, \mathcal{A}}$ where $\mathbf{f}_{\nu, \mathcal{A}}=\mathbf{f}_{\nu} \cap \mathbf{f}_{\mathcal{A}}$. Let $\mathbf{f}_{A}=A \otimes_{\mathcal{A}} \mathbf{f}_{\mathcal{A}}$, an $A$-algebra. We have $\mathbf{f}_{A}=\bigoplus_{\nu \in \mathbf{Z}[I]} \mathbf{f}_{\nu, A}$ where $\mathbf{f}_{\nu, A}=A \otimes_{\mathcal{A}} \mathbf{f}_{\nu, \mathcal{A}}$.

Let $\mathbf{B}$ be the canonical basis of $\mathbf{f}$ (see [L1, 14.4]). Note that $\mathbf{B}$ is also an $\mathcal{A}$-basis of $\mathbf{f}_{\mathcal{A}}$. If $b \in \mathbf{B}$, we shall denote the element $1 \otimes_{\mathcal{A}} b \in A \otimes_{\mathcal{A}} \mathbf{f}_{\mathcal{A}}=\mathbf{f}_{A}$ again by $b$. Thus $\mathbf{B}$ can be viewed also as an $A$-basis of $\mathbf{f}_{A}$.

1.4. For any $n \in \mathbf{Z}, t \in \mathbf{N}$ and $i \in I$ we define $\left[\begin{array}{l}n \\ t\end{array}\right]_{i} \in \mathcal{A}$ as in [L1, 1.3.1, 1.1.2].

Let $\dot{\mathbf{U}}_{A}$ be the $A$-algebra generated by the symbols $x^{+} 1_{\zeta} x^{\prime-}, x^{-} 1_{\zeta} x^{\prime+}$ with $x \in \mathbf{f}_{\nu, A}, x^{\prime} \in \mathbf{f}_{\nu^{\prime}, A}$ for various $\nu, \nu^{\prime}$ and $\zeta \in X$; these symbols are subject to the following relations:

$\mathbf{f}_{A} \rightarrow \dot{\mathbf{U}}_{A}, x \mapsto x^{ \pm} 1_{\zeta}$ is $A$-linear for any $\zeta \in X$;

$\theta_{i}^{(n)+} 1_{\zeta} \theta_{j}^{(m)-}=\theta_{j}^{(m)-} 1_{\zeta} \theta_{i}^{(n)+}$ if $m, n \in \mathbf{N}, \zeta \in X, i \neq j$;

$\theta_{i}^{(n) \pm} 1_{\mp \zeta} \theta_{i}^{(m) \mp}=\sum_{t \in \mathbf{N}}\left[m+n-\langle i, \zeta\rangle t_{i}\right] \theta_{i}^{(m-t) \mp} 1_{\mp \zeta \pm(a+b-t) i^{\prime}} \theta_{i}^{(n-t) \pm}$ if $m, n \in$ $\mathbf{N}, \zeta \in X, i \in I$ 


$$
\begin{aligned}
& x^{ \pm} 1_{\zeta}=1_{\zeta \pm \nu} x^{ \pm} \text {if } x \in \mathbf{f}_{\nu, A}, \zeta \in X ; \\
& \left(x^{ \pm} 1_{\zeta}\right)\left(1_{\zeta^{\prime}} x^{\prime \mp}\right)=\delta_{\zeta, \zeta^{\prime}} x^{ \pm} 1_{\zeta} x^{\prime \mp} \text { if } x, x^{\prime} \in \mathbf{f}_{A}, \zeta \in X ; \\
& \left(x^{ \pm} 1_{\zeta}\right)\left(1_{\zeta^{\prime}} x^{\prime \pm}\right)=\delta_{\zeta, \zeta^{\prime}} 1_{\zeta \pm \nu}\left(x x^{\prime}\right)^{ \pm} \text {if } x \in \mathbf{f}_{\nu, A}, x^{\prime} \in \mathbf{f}_{A}, \zeta \in X .
\end{aligned}
$$

If $x$ or $x^{\prime}$ in $x^{+} 1_{\zeta} x^{\prime-}$ or $x^{-} 1_{\zeta} x^{\prime+}$ is 1 , we omit writing it.

(See [L1, 31.1.1, 31.1.3].) Note that the two $A$-linear maps $\mathbf{f}_{A} \otimes_{A} A[X] \otimes_{A} \mathbf{f}_{A} \rightarrow \dot{\mathbf{U}}_{A}$, $x \otimes \lambda \otimes x^{\prime} \mapsto x^{+} 1_{\lambda} x^{\prime-}$ and $x \otimes \lambda \otimes x^{\prime} \mapsto x^{-} 1_{\lambda} x^{\prime+}$ are isomorphisms of $A$-modules. Hence we have canonically $\dot{\mathbf{U}}_{A}=A \otimes_{\mathcal{A}} \dot{\mathbf{U}}_{\mathcal{A}}$ as $A$-algebras.

Now $\dot{\mathbf{U}}_{A}$ does not have 1 in general; instead it has a family of elements $1_{\lambda}(\lambda \in X)$ such that $1_{\lambda} 1_{\lambda^{\prime}}=\delta_{\lambda, \lambda^{\prime}}$ for any $\lambda, \lambda^{\prime}$ in $X$ and such that $\dot{\mathbf{U}}_{A}=\sum_{\lambda, \lambda^{\prime} \in X} 1_{\lambda} \dot{\mathbf{U}}_{A} 1_{\lambda^{\prime}}$ (necessarily a direct sum).

In the case where $A=\mathbf{Q}(v)$ we write $\dot{\mathbf{U}}$ instead of $\dot{\mathbf{U}}_{A}$. Now the obvious map $\dot{\mathbf{U}}_{\mathcal{A}} \rightarrow \dot{\mathbf{U}}$ induced by the inclusion $\mathcal{A} \subset \mathbf{Q}(v)$ is injective and identifies $\dot{\mathbf{U}}_{\mathcal{A}}$ with an $\mathcal{A}$-subalgebra of $\dot{\mathbf{U}}$. For any $\lambda, \lambda^{\prime}, \lambda_{1}, \lambda_{2}, \lambda_{1}^{\prime}, \lambda_{2}^{\prime}$ in $X$ we define an $A$-linear map

$$
\Delta_{\lambda, \lambda^{\prime}, \lambda_{1}, \lambda_{1}^{\prime}, \lambda_{2}, \lambda_{2}^{\prime}}: 1_{\lambda} \dot{\mathbf{U}}_{A} 1_{\lambda^{\prime}} \rightarrow\left(1_{\lambda_{1}} \dot{\mathbf{U}}_{A} 1_{\lambda_{1}^{\prime}}\right) \otimes_{A}\left(1_{\lambda_{2}} \dot{\mathbf{U}}_{A} 1_{\lambda_{2}^{\prime}}\right)
$$

as follows: if $\lambda=\lambda_{1}+\lambda_{2}, \lambda^{\prime}=\lambda_{1}^{\prime}+\lambda_{2}^{\prime}$, it is the map obtained by applying $A \otimes_{\mathcal{A}}$ ? to the $\mathcal{A}$-linear map at the end of [L1, 23.2.3]; otherwise, it is 0 . We define an algebra isomorphism $S$ from $\dot{\mathbf{U}}_{A}$ to $\dot{\mathbf{U}}_{A}$ with opposed multiplication as follows: if $A=\mathbf{Q}(v)$, $S$ is as in [L1, 23.1.6]; next, $S: \dot{\mathbf{U}}_{\mathcal{A}} \rightarrow \dot{\mathbf{U}}_{\mathcal{A}}$ is the retriction of $S: \dot{\mathbf{U}} \rightarrow \dot{\mathbf{U}}$ to $\dot{\mathbf{U}}_{\mathcal{A}}$; for general $A, S$ is obtained by applying $A \otimes_{\mathcal{A}}$ ? to the $\mathcal{A}$-linear map $S: \dot{\mathbf{U}}_{\mathcal{A}} \rightarrow \dot{\mathbf{U}}_{\mathcal{A}}$. Let $\omega: \dot{\mathbf{U}}_{A} \rightarrow \dot{\mathbf{U}}_{A}$ be the algebra automorphism (with square 1) defined in [L1, $31.1 .4]$.

1.5. Let $\dot{\mathbf{B}}$ be the canonical basis of $\dot{\mathbf{U}}_{\mathcal{A}}$ (see [L1, 25.2]). If $b \in \dot{\mathbf{B}}$, we shall denote the element $1 \otimes_{\mathcal{A}} b \in A \otimes_{\mathcal{A}} \dot{\mathbf{U}}_{\mathcal{A}}=\dot{\mathbf{U}}_{A}$ again by $b$. Thus $\dot{\mathbf{B}}$ can be viewed also as an $A$-basis of $\dot{\mathbf{U}}_{A}$.

Note that $1_{\lambda} \in \dot{\mathbf{B}}$ for any $\lambda \in X$. We have $\dot{\mathbf{B}}=\bigsqcup_{\lambda, \lambda^{\prime} \in X}\left(\dot{\mathbf{B}} \cap\left(1_{\lambda} \dot{\mathbf{U}}_{A} 1_{\lambda^{\prime}}\right)\right)$. For $a, b$ in $\dot{\mathbf{B}}$ we write $a b=\sum_{c \in \mathbf{B}} m_{a, b}^{c} c$ ( $a b$ is the product in $\dot{\mathbf{U}}_{A}$ and $m_{a, b}^{c} \in A$ is 0 for all but finitely many $c$ ). For $a, b, c$ in $\dot{\mathbf{B}}$ we define $\hat{m}_{c}^{a, b} \in A$ by the following requirement: for any $\lambda, \lambda^{\prime}, \lambda_{1}, \lambda_{1}^{\prime}, \lambda_{2}, \lambda_{2}^{\prime}$ in $X$ and any $c \in \dot{\mathbf{B}} \cap\left(1_{\lambda} \dot{\mathbf{U}}_{A} 1_{\lambda^{\prime}}\right)$ we have $\Delta_{\lambda, \lambda^{\prime}, \lambda_{1}, \lambda_{1}^{\prime}, \lambda_{2}, \lambda_{2}^{\prime}} c=\sum_{a, b} \hat{m}_{c}^{a, b} a \otimes b$ where $a$ runs over $\dot{\mathbf{B}} \cap\left(1_{\lambda_{1}} \dot{\mathbf{U}}_{A} 1_{\lambda_{1}^{\prime}}\right), b$ runs over $\dot{\mathbf{B}} \cap\left(1_{\lambda_{2}} \dot{\mathbf{U}}_{A} 1_{\lambda_{2}^{\prime}}\right)$ (in the last sum $\hat{m}_{c}^{a, b}$ is 0 for all but finitely many $(a, b)$ ).

For any $a \in \dot{\mathbf{B}}$ we have $S(a)=s_{a} \underline{a}$ where $a \mapsto \underline{a}$ is an involution of $\dot{\mathbf{B}}$ and $s_{a}$ is \pm a power of $v$ with $s_{\underline{a}}=s_{a}$ (see [L1, 23.1.7, 26.3.2]). We have $s_{1_{\lambda}}=1, \underline{1_{\lambda}}=1_{-\lambda}$ for $\lambda \in X$ (see [L1, 23.1.6]). For any $a \in \dot{\mathbf{B}}$ we have $\omega(a)=e_{a} a^{!}$where $\bar{a} \mapsto a^{!}$is an involution of $\dot{\mathbf{B}}$ and $e_{a}= \pm 1$ (see [L1, 26.3.2]). We have $e_{1_{\lambda}}=1,\left(1_{\lambda}\right)^{!}=1_{-\lambda}$ for $\lambda \in X$.

Note that the quantities $m_{a, b}^{c}, \hat{m}_{c}^{a, b}, s_{a}, e_{a}$ (in $A$ ) are the images of the corresponding quantities in $\mathcal{A}$ under the given homomorphism $\mathcal{A} \rightarrow A$ and the involutions $a \mapsto \underline{a}, a \mapsto a$ ! are independent of $A$.

As in [L1, 25.4] for any $a, b, d, e$ in $\dot{\mathbf{B}}$ we have

(i) $\sum_{c} m_{a, b}^{c} m_{c, d}^{e}=\sum_{c} m_{a, c}^{e} m_{b, d}^{c}$;

(ii) $\sum_{c} \hat{m}_{c}^{a, b} \hat{m}_{e}^{c, d}=\sum_{c} \hat{m}_{e}^{a, c} \hat{m}_{c}^{b, d}$;

(iii) $\sum_{c} m_{a, b}^{c} \hat{m}_{c}^{e, d}=\sum_{a^{\prime}, b^{\prime}, c^{\prime}, d^{\prime}} \hat{m}_{a}^{a^{\prime}, b^{\prime}} \hat{m}_{b}^{c^{\prime}, d^{\prime}} m_{a^{\prime}, c^{\prime}}^{e} m_{b^{\prime}, d^{\prime}}^{d}$;

(iv) $\hat{m}_{1_{\lambda}}^{a, b}=1$ if $a=1_{\lambda^{\prime}}, b=1_{\lambda^{\prime \prime}}, \lambda^{\prime}+\lambda^{\prime \prime}=\lambda$ and $\hat{m}_{1_{\lambda}}^{a, b}=0$, otherwise. 
From the definitions we have for any $a, b, c$ in $\dot{\mathbf{B}}$ :

(v) $s_{c} m_{a, b}^{c}=s_{a} s_{b} m_{\underline{b}, a}^{c}$;

(vi) $s_{c} \hat{m}_{\underline{\underline{b}}}^{\underline{\underline{b}} \underline{a}}=s_{a} s_{b} \hat{m}_{c}^{a, b}$

(vii) $e_{c} m_{a, b}^{c}=e_{a} e_{b} m_{a^{\prime}, b^{!}}^{c^{\prime}}$;

(viii) $e_{c} \hat{m}_{c^{!}}^{b^{!}, a^{!}}=e_{a} e_{b} \hat{m}_{c}^{a, b}$;

(ix) $\sum_{\lambda \in X} m_{a, 1_{\lambda}}^{c}=\sum_{\lambda \in X} m_{1_{\lambda}, a}^{c}=\delta_{a, c}$;

(x) $\hat{m}_{c}^{a, 1_{0}}=\hat{m}_{c}^{1_{0}, a}=\delta_{a, c}$;

(xi) $\sum_{d, e \in \dot{\mathbf{B}}} \hat{m}_{a}^{d, e} m_{d, \tilde{e}}^{b} s_{e}=\sum_{d, e \in \dot{\mathbf{B}}} \hat{m}_{a}^{d, e} m_{\tilde{d}, e}^{b} s_{d}$ is 1 if $b=1_{\lambda}, a=1_{0}$ and is 0 otherwise;

(xii) $m_{a, b}^{1_{0}}=\delta_{a, 1_{0}} \delta_{b, 1_{0}}$;

(xiii) $\hat{m}_{c}^{a, b}=\hat{m}_{c}^{b, a}$ in $A$ if $v=1$ in $A$.

1.6. Let $\mathfrak{C}_{A}$ be the category whose objects are $\dot{\mathbf{U}}_{A}$-modules $M$ which are unital in the following sense: for any $z \in M$ we have $1_{\lambda} z=0$ for all but finitely many $\lambda \in X$ and $\sum_{\lambda \in X} 1_{\lambda} z=z$ and which are finitely generated as an $A$-module. (A unital $\dot{\mathbf{U}}_{A}$-module $M$ is also an $A$-module by $a: z \mapsto \sum_{\lambda \in X}\left(a 1_{\lambda}\right) z$ (all but finitely many terms of the last sum are 0 ). A morphism in $\mathfrak{C}_{A}$ is a $\dot{\mathbf{U}}_{A}$-linear map. When $A=\mathbf{Q}(v)$, we write $\mathfrak{C}$ instead of $\mathfrak{C}_{A}$.

Let $M, M^{\prime} \in \mathfrak{C}_{A}$. The tensor product $M \otimes_{A} M^{\prime}$ will be regarded as a $\dot{\mathbf{U}}_{A^{-}}$-module by the rule $c\left(z \otimes z^{\prime}\right)=\sum_{a, b \in \dot{\mathbf{B}}} \hat{m}_{c}^{a, b} a z \otimes b z^{\prime}$ where $c \in \dot{\mathbf{B}}, z \in M, z^{\prime} \in M^{\prime}$. (All but finitely many terms of the last sum are 0.) The fact that the rule above defines an $\dot{\mathbf{U}}_{A}$-module structure follows from 1.5(iii). We have $M \otimes_{A} M^{\prime} \in \mathfrak{C}_{A}$. This makes $\mathfrak{C}_{A}$ into a monoidal tensor category.

For any $M, M^{\prime}$ in $\mathfrak{C}_{A}$ let ${ }_{f} \mathcal{R}_{M, M^{\prime}}: M^{\prime} \otimes M \rightarrow M \otimes M^{\prime}$ be the isomorphism in $\mathfrak{C}_{A}$ defined in [L1, 32.1.5] in terms of a fixed function $f: X \times X \rightarrow \mathbf{Z}$ as in [L1, 32.1.3]. (In the formula for ${ }_{f} \mathcal{R}_{M, M^{\prime}}$ in [L1, 32.1.4] we interpret $b^{-} m, b^{+} m^{\prime}$ as $\sum_{\lambda} b^{-} 1_{\lambda} m$, $\sum_{\lambda} b^{+} 1_{\lambda} m^{\prime}$.)

To any object $M \in \mathfrak{C}_{A}$ we associate a new object ${ }^{\omega} M \in \mathfrak{C}_{A}$ with the same underlying $A$-module as $M$ and such that for any $u \in \dot{\mathbf{U}}_{A}$, the operator $u$ on ${ }^{\omega} M$ coincides with the operator $\omega(u)$ on $M$.

For any $\lambda \in X^{+}$let $\Lambda_{\lambda}=\mathbf{f} / \mathcal{T}_{\lambda}$ where $\mathcal{T}_{\lambda}=\sum_{i} \mathbf{f} \theta_{i}^{\langle i, \lambda\rangle+1}$. Let $\eta_{\lambda}=1+\mathcal{T}_{\lambda}$. We regard $\Lambda_{\lambda}$ as an object of $\mathfrak{C}$ in which $\left(\theta_{i}^{+} 1_{\lambda^{\prime}}\right) \eta_{\lambda}=0$ for $i \in I, \lambda^{\prime} \in X$ and $\left(x^{-} 1_{\lambda^{\prime}}\right) \eta_{\lambda}=\delta_{\lambda, \lambda^{\prime}} x+\mathcal{T}_{\lambda}$ for $x \in \mathbf{f}, \lambda^{\prime} \in X$. We have $\Lambda_{\lambda} \in \mathfrak{C}([\mathrm{L} 1,6.3 .4,23.1 .4])$.

Let $\Lambda_{\lambda, \mathcal{A}}=\dot{\mathbf{U}}_{\mathcal{A}} \eta_{\lambda}$. This is an $\mathcal{A}$-lattice in $\Lambda_{\lambda}$ and $\Lambda_{\lambda, \mathcal{A}} \in \mathfrak{C}_{\mathcal{A}}$. Note that $\eta_{\lambda} \in \Lambda_{\lambda, \mathcal{A}}$.

Let $\Lambda_{\lambda, A}=A \otimes_{\mathcal{A}} \Lambda_{\lambda, \mathcal{A}}$. By extension of scalars $\Lambda_{\lambda, A}$ becomes a $\dot{\mathbf{U}}_{A^{-} \text {-module. }}$ We have $\Lambda_{\lambda, A} \in \mathfrak{C}_{A}$. We write $\eta_{\lambda}$ instead of $1 \otimes_{\mathcal{A}} \eta_{\lambda} \in A \otimes_{\mathcal{A}} \Lambda_{\lambda, \mathcal{A}}=L_{\lambda, A}$. Now $\eta_{\lambda} \in \Lambda_{\lambda, A}$ regarded as an element of ${ }^{\omega} \Lambda_{\lambda, A}$ will be denoted by $\xi_{-\lambda}$.

For $\lambda \in X^{+}$there is a unique subset $\mathbf{B}_{\lambda}$ of $\mathbf{B}$ such that $b \mapsto\left(b^{-} 1_{\lambda}\right) \eta_{\lambda}$ is a bijection of $\mathbf{B}_{\lambda}$ onto a basis $\underline{\mathbf{B}}_{\lambda}$ of $\Lambda_{\lambda, A}$; for $b \in \mathbf{B}-\mathbf{B}_{\lambda}$ we have $\left(b^{-} 1_{\lambda}\right) \eta_{\lambda}=0$. There is a unique subset $\mathbf{B}_{\lambda}^{\prime}$ of $\mathbf{B}$ such that $b \mapsto\left(b^{+} 1_{-\lambda}\right) \xi_{-\lambda}$ is a bijection of $\mathbf{B}_{\lambda}^{\prime}$ onto $\underline{\mathbf{B}}_{\lambda}$; for $b \in \mathbf{B}-\mathbf{B}_{\lambda}^{\prime}$ we have $\left(b^{+} 1_{-\lambda}\right) \xi_{-\lambda}=0$.

Note that $\mathbf{B}_{\lambda}, \mathbf{B}_{\lambda}^{\prime}$ are independent of $A$.

1.7. For $\lambda \in X^{+}$let $\dot{\mathbf{U}}^{\geq \lambda}$ be the set of all $u \in \dot{\mathbf{U}}$ such that for any $\lambda^{\prime} \in X^{+}$such that $\lambda^{\prime} \nsucceq \lambda$ we have $\left.u\right|_{\Lambda_{\lambda^{\prime}}}=0$ (a two-sided ideal of $\dot{\mathbf{U}}$ ). By [L1, 29.1], $\dot{\mathbf{U}} \geq \lambda \cap \dot{\mathbf{B}}$ is a basis of $\dot{\mathbf{U}}^{\geq \lambda}$ and there is a unique partition $\dot{\mathbf{B}}=\bigsqcup_{\lambda \in X^{+}} \dot{\mathbf{B}}[\lambda]$ such that for any 
$\lambda \in X^{+}$we have $\dot{\mathbf{U}} \geq \lambda \cap \dot{\mathbf{B}}=\bigsqcup_{\lambda^{\prime} \in X^{+} ; \lambda^{\prime} \geq \lambda} \dot{\mathbf{B}}\left[\lambda^{\prime}\right]$. By [L1, 29.1.6], $\dot{\mathbf{B}}[\lambda]$ is finite for any $\lambda \in X^{+}$.

Lemma 1.8. For any $c \in \dot{\mathbf{B}}$, the set $\left\{(a, b) \in \dot{\mathbf{B}} \times \dot{\mathbf{B}} ; m_{a, b}^{c} \in A-\{0\}\right\}$ is finite.

We may assume that $A=\mathcal{A}$. Let $c \in \dot{\mathbf{B}}$. We have $c \in \dot{\mathbf{B}}[\lambda]$ for a unique $\lambda \in X^{+}$. Let $(a, b) \in \dot{\mathbf{B}} \times \dot{\mathbf{B}}$ be such that $m_{a, b}^{c} \neq 0$. We have $a \in \dot{\mathbf{B}}\left[\lambda^{\prime}\right], b \in \dot{\mathbf{B}}\left[\lambda^{\prime \prime}\right]$ for some $\lambda^{\prime}, \lambda^{\prime \prime}$ in $X^{+}$. We have $a \in \dot{\mathbf{U}} \geq \lambda^{\prime}$. Since $\dot{\mathbf{U}} \geq \lambda^{\prime} \dot{\mathbf{U}} \subset \dot{\mathbf{U}} \geq \lambda^{\prime}$, we have $a b \in \dot{\mathbf{U}} \geq \lambda^{\prime}$. Since $c$ appears with non-zero coefficient in $a b$ and $\dot{\mathbf{U}} \geq \lambda^{\prime} \cap \dot{\mathbf{B}}$ is a basis of $\dot{\mathbf{U}} \geq \lambda^{\prime}$, we have $c \in$ $\dot{\mathbf{U}} \geq \lambda^{\prime} \cap \dot{\mathbf{B}}$, that is, $c \in \bigcup_{\lambda_{1} \in X^{+} ; \lambda_{1} \geq \lambda^{\prime}} \dot{\mathbf{B}}\left[\lambda_{1}\right]$. Thus $\lambda \geq \lambda^{\prime}$. Similarly, $\lambda \geq \lambda^{\prime \prime}$. Thus $\left\{(a, b) \in \dot{\mathbf{B}} \times \dot{\mathbf{B}} ; m_{a, b}^{c} \neq 0\right\}$ is contained in $\bigsqcup_{\left(\lambda^{\prime}, \lambda^{\prime \prime}\right) \in X^{+} \times X^{+} ; \lambda \geq \lambda^{\prime}, \lambda>\lambda^{\prime \prime}} \dot{\mathbf{B}}\left[\lambda^{\prime}\right] \times \dot{\mathbf{B}}\left[\lambda^{\prime \prime}\right]$; this is a finite set since $\left\{\left(\lambda^{\prime}, \lambda^{\prime \prime}\right) \in X^{+} \times X^{+} ; \lambda \geq \lambda^{\prime}, \lambda \geq \lambda^{\prime \prime}\right\}$ is finite and each $\dot{\mathbf{B}}\left[\lambda^{\prime}\right] \times \dot{\mathbf{B}}\left[\lambda^{\prime \prime}\right]$ is finite. The lemma is proved.

1.9. For any $\lambda, \lambda^{\prime}$ in $X^{+}$there is a unique (finite) subset $\dot{\mathbf{B}}_{\lambda, \lambda^{\prime}}$ of $\dot{\mathbf{B}}$ such that $a \mapsto a\left(\xi_{-\lambda} \otimes \eta_{\lambda^{\prime}}\right)$ defines a bijection of $\dot{\mathbf{B}}_{\lambda, \lambda^{\prime}}$ onto an $A$-basis $\underline{\mathbf{B}}_{\lambda, \lambda^{\prime}}$ of ${ }^{\omega} \Lambda_{\lambda, A} \otimes_{A} \Lambda_{\lambda^{\prime}, A}$; moreover $a\left(\xi_{-\lambda} \otimes_{A} \eta_{\lambda^{\prime}}\right)=0$ for any $a \in \mathbf{B}-\dot{\mathbf{B}}_{\lambda, \lambda^{\prime}}$. This property appears in L1, 25.2.1] in the case where $A=\mathbf{Q}(v)$ but it is then automatically true for general $A$. Note that the subset $\dot{\mathbf{B}}_{\lambda, \lambda^{\prime}}$ of $\dot{\mathbf{B}}$ is independent of $A$. We have $\dot{\mathbf{B}}=\bigcup_{\lambda, \lambda^{\prime} \in X^{+}} \dot{\mathbf{B}}_{\lambda, \lambda^{\prime}}$.

1.10. We show:

(a) If $M \in \mathfrak{C}_{A}$, then $\mathfrak{A}:=\{a \in \dot{\mathbf{B}} ; a M \neq 0\}$ is finite.

Let $z_{1}, z_{2}, \ldots, z_{r}$ be a set of generators of the $A$-module $M$ such that each $z_{j}$ is contained in $1_{\zeta_{j}} M$ for some $\zeta_{j} \in X$. As in the proof of [L1, 31.2.7], for $j \in[1, r]$ we can find $\lambda_{j}, \lambda_{j}^{\prime} \in X^{+}$such that $\lambda_{j}^{\prime}-\lambda_{j}=\zeta_{j}$ and a morphism $\phi_{j}:{ }^{\omega} \Lambda_{\lambda_{j}, A} \otimes{ }_{A} \Lambda_{\lambda_{j}^{\prime}, A} \rightarrow M$ in $\mathfrak{C}_{A}$ such that $\phi\left(\xi_{-\lambda_{j}} \otimes \eta_{\lambda_{j}^{\prime}}\right)=z_{j}$. If $a \in \mathfrak{A}$, then for some $j$ we have $a z_{j} \neq 0$, hence $a\left(\xi_{-\lambda_{j}} \otimes \eta_{\lambda_{j}^{\prime}}\right) \neq 0$ in ${ }^{\omega} \Lambda_{\lambda_{j}, A} \otimes_{A} \Lambda_{\lambda_{j}^{\prime}, A}$. Thus we have $a \in \dot{\mathbf{B}}_{\lambda_{j}, \lambda_{j}^{\prime}}$. We see that $\mathfrak{A} \subset \bigcup_{j \in[1, r]} \dot{\mathbf{B}}_{\lambda_{j}, \lambda_{j}^{\prime}}$. Thus $\mathfrak{A}$ is finite.

1.11. Let $\hat{\mathbf{U}}_{A}$ be the $A$-module consisting of all formal linear combinations

$$
\sum_{a \in \dot{\mathbf{B}}} n_{a} a
$$

with $n_{a} \in A$. We define an $A$-algebra structure on $\hat{\mathbf{U}}_{A}$ by

$$
\left(\sum_{a \in \dot{\mathbf{B}}} n_{a} a\right)\left(\sum_{b \in \dot{\mathbf{B}}} \tilde{n}_{b} b\right)=\sum_{c \in \dot{\mathbf{B}}} r_{c} c
$$

where $r_{c}=\sum_{(a, b) \in \dot{\mathbf{B}} \times \dot{\mathbf{B}}} m_{a, b}^{c} n_{a} \tilde{n}_{b}$. The last sum is well defined since by Lemma 1.8 it has only finitely many non-zero terms. This algebra structure is associative by 1.5(i) and has a unit element $1=\sum_{\lambda \in X} 1_{\lambda}$. We have an imbedding of $A$-algebras $\dot{\mathbf{U}}_{A} \subset \hat{\mathbf{U}}_{A}$ under which $b \in \dot{\mathbf{B}} \subset \dot{\mathbf{U}}_{A}$ corresponds to $\sum_{a \in \dot{\mathbf{B}}} \delta_{a, b} a \in \hat{\mathbf{U}}_{A}$.

Let $\hat{\mathbf{U}}_{A}^{(2)}$ be the $A$-module consisting of all formal linear combinations

$$
\sum_{\left(a, a^{\prime}\right) \in \dot{\mathbf{B}} \times \dot{\mathbf{B}}} n_{a, a^{\prime}} a \otimes a^{\prime}
$$

with $n_{a, a^{\prime}} \in A$. We define an $A$-algebra structure on $\hat{\mathbf{U}}_{A}^{(2)}$ by

$$
\left(\sum_{\left(a, a^{\prime}\right) \in \dot{\mathbf{B}} \times \dot{\mathbf{B}}} n_{a, a^{\prime}} a \otimes a^{\prime}\right)\left(\sum_{\left(b, b^{\prime}\right) \in \dot{\mathbf{B}} \times \dot{\mathbf{B}}} \tilde{n}_{b, b^{\prime}} b \otimes b^{\prime}\right)=\sum_{\left(c, c^{\prime}\right) \in \dot{\mathbf{B}} \times \dot{\mathbf{B}}} r_{c, c^{\prime}} c \otimes c^{\prime}
$$


where $r_{c, c^{\prime}}=\sum_{a, a^{\prime}, b, b^{\prime}} m_{a, b}^{c} m_{a^{\prime}, b^{\prime}}^{c^{\prime}} n_{a, a^{\prime}} \tilde{n}_{b, b^{\prime}}$. (Again this sum is well defined by 1.8.) This algebra is associative by $1.5(\mathrm{i})$ with unit element $1=\sum_{\lambda, \lambda^{\prime} \in X} 1_{\lambda} \otimes 1_{\lambda^{\prime}}$. Note that $\hat{\mathbf{U}}_{A}^{(2)}$ is associated to the direct sum of two copies of our root datum in the same way that $\hat{\mathbf{U}}_{A}$ is associated to our root datum. For $\xi=\sum_{a \in \dot{\mathbf{B}}} n_{a} a \in \hat{\mathbf{U}}_{A}$, $\xi^{\prime}=\sum_{a^{\prime} \in \dot{\mathbf{B}}} \tilde{n}_{a^{\prime}} a^{\prime} \in \hat{\mathbf{U}}_{A}$, we set $\xi \otimes \xi^{\prime}=\sum_{\left(a, a^{\prime}\right) \in \dot{\mathbf{B}} \times \dot{\mathbf{B}}} n_{a} \tilde{n}_{a^{\prime}} a \otimes a^{\prime} \in \hat{\mathbf{U}}^{(2)}$. Note that $\xi, \xi^{\prime} \mapsto \xi \otimes \xi^{\prime}$ induces an $A$-algebra homomorphisms $\hat{\mathbf{U}}_{A} \otimes_{A} \hat{\mathbf{U}}_{A} \rightarrow \hat{\mathbf{U}}_{A}^{(2)}$. We define an $A$-linear map $\Delta: \dot{\mathbf{U}}_{A} \rightarrow \hat{\mathbf{U}}_{A}^{(2)}$ by

$$
c \mapsto \sum_{(a, b) \in \dot{\mathbf{B}} \times \dot{\mathbf{B}}} \hat{m}_{c}^{a, b} a \otimes b
$$

for any $c \in \dot{\mathbf{B}}$. By 1.5(iii), $\Delta$ is an $A$-algebra homomorphism.

We define an $A$-linear map $\mathbf{m}: \hat{\mathbf{U}}_{A}^{(2)} \rightarrow \hat{\mathbf{U}}_{A}$ by

$$
\sum_{\left(a, a^{\prime}\right) \in \dot{\mathbf{B}} \times \dot{\mathbf{B}}} n_{a, a^{\prime}} a \otimes a^{\prime} \mapsto \sum_{b \in \dot{\mathbf{B}}}\left(\sum_{\left(a, a^{\prime}\right) \in \dot{\mathbf{B}} \times \dot{\mathbf{B}}} n_{a, a^{\prime}} m_{a, a^{\prime}}^{b}\right) b .
$$

This is well defined, by 1.8. In particular, if $\xi, \xi^{\prime} \in \hat{\mathbf{U}}_{A}$ we have $\mathbf{m}\left(\xi \otimes \xi^{\prime}\right)=\xi \xi^{\prime}$ (product in $\hat{\mathbf{U}}_{A}$ ) where $\xi \otimes \xi^{\prime}$ is regarded as an element of $\hat{\mathbf{U}}^{(2)}$ as above.

We define an $A$-linear map $S: \hat{\mathbf{U}}_{A} \rightarrow \hat{\mathbf{U}}_{A}$ by $\sum_{a \in \dot{\mathbf{B}}} n_{a} a \mapsto \sum_{a \in \dot{\mathbf{B}}} s_{a} n_{a} \underline{a}$ and $A$-linear maps $S^{(2)}: \hat{\mathbf{U}}_{A}^{(2)} \rightarrow \hat{\mathbf{U}}_{A}^{(2)}, S^{(0,1)}: \hat{\mathbf{U}}_{A}^{(2)} \rightarrow \hat{\mathbf{U}}_{A}^{(2)}$ by

$$
\begin{aligned}
& S^{(2)}: \sum_{\left(a, a^{\prime}\right) \in \dot{\mathbf{B}} \times \dot{\mathbf{B}}} n_{a, a^{\prime}} a \otimes a^{\prime} \mapsto \sum_{\left(a, a^{\prime}\right) \in \dot{\mathbf{B}} \times \dot{\mathbf{B}}} s_{a} s_{a^{\prime}} n_{a, a^{\prime}} \underline{a} \otimes \underline{a}^{\prime}, \\
& S^{(0,1)}: \sum_{\left(a, a^{\prime}\right) \in \dot{\mathbf{B}} \times \dot{\mathbf{B}}} n_{a, a^{\prime}} a \otimes a^{\prime} \mapsto \sum_{\left(a, a^{\prime}\right) \in \dot{\mathbf{B}} \times \dot{\mathbf{B}}} s_{a^{\prime}} n_{a, a^{\prime}} a \otimes \underline{a}^{\prime} .
\end{aligned}
$$

In particular for $\xi, \xi^{\prime}$ in $\hat{\mathbf{U}}_{A}$ we have $S^{(2)}\left(\xi \otimes \xi^{\prime}\right)=S(\xi) \otimes S\left(\xi^{\prime}\right), S^{(0,1)}\left(\xi \otimes \xi^{\prime}\right)=$ $\xi \otimes S\left(\xi^{\prime}\right)$.

We define an $A$-linear map $\omega: \hat{\mathbf{U}}_{A} \rightarrow \hat{\mathbf{U}}_{A}$ by $\sum_{a \in \dot{\mathbf{B}}} n_{a} a \mapsto \sum_{a \in \dot{\mathbf{B}}} e_{a} n_{a} a^{!}$and an $A$-linear map $\omega^{(2)}: \hat{\mathbf{U}}_{A}^{(2)} \rightarrow \hat{\mathbf{U}}_{A}^{(2)}$ by

$$
\sum_{\left(a, a^{\prime}\right) \in \dot{\mathbf{B}} \times \dot{\mathbf{B}}} n_{a, a^{\prime}} a \otimes a^{\prime} \mapsto \sum_{\left(a, a^{\prime}\right) \in \dot{\mathbf{B}} \times \dot{\mathbf{B}}} e_{a} e_{a^{\prime}} n_{a, a^{\prime}} a^{!} \otimes a^{\prime !} .
$$

In particular for $\xi, \xi^{\prime}$ in $\hat{\mathbf{U}}_{A}$ we have $\omega^{(2)}\left(\xi \otimes \xi^{\prime}\right)=\omega(\xi) \otimes \omega\left(\xi^{\prime}\right)$.

For $\xi, \xi^{\prime}$ in $\hat{\mathbf{U}}_{A}$ we have $S\left(\xi \xi^{\prime}\right)=S\left(\xi^{\prime}\right) S(\xi), \omega\left(\xi \xi^{\prime}\right)=\omega(\xi) \omega\left(\xi^{\prime}\right)$ (see 1.5(v), (vii)).

We define an $A$-linear map $\epsilon: \hat{\mathbf{U}}_{A} \rightarrow A$ by $\sum_{a \in \dot{\mathbf{B}}} n_{a} a \mapsto n_{1_{0}}$. This is a homomorphism of $A$-algebras preserving 1 , by 1.5 (xii).

1.12. Let $M \in \mathfrak{C}_{A}$. We define an $A$-linear map $\hat{\mathbf{U}}_{A} \rightarrow \operatorname{Hom}_{A}(M, M)$ by $\sum_{a \in \dot{\mathbf{B}}} n_{a} a:$ $z \mapsto \sum_{a \in \dot{\mathbf{B}}} n_{a} a z$. The last sum is well defined since, by 1.10(a), all but finitely many of its terms are 0 . This defines a structure of left $\hat{\mathbf{U}}_{A^{-}}$-module on $M$ extending the $\dot{\mathbf{U}}_{A}$-module structure. Note that $1 \in \hat{\mathbf{U}}_{A}$ acts as 1 on $M$.

Similarly, if $M \in \mathfrak{C}_{A}, M^{\prime} \in \mathfrak{C}_{A}$, then the obvious $\dot{\mathbf{U}}_{A} \otimes \dot{\mathbf{U}}_{A}$-module structure on $M \otimes_{A} M^{\prime}$ extends to a $\hat{\mathbf{U}}_{A}^{(2)}$-module structure on $M \otimes_{A} M^{\prime}$ defined by the $A$-algebra 


$$
\begin{aligned}
\operatorname{map} \hat{\mathbf{U}}_{A}^{(2)} \rightarrow & \operatorname{Hom}_{A}\left(M \otimes_{A} M^{\prime}, M \otimes_{A} M^{\prime}\right), \\
& \sum_{\left(a, a^{\prime}\right) \in \dot{\mathbf{B}} \times \dot{\mathbf{B}}} n_{a, a^{\prime}} a \otimes a^{\prime}: z \otimes z^{\prime} \mapsto \sum_{\left(a, a^{\prime}\right) \in \dot{\mathbf{B}} \times \dot{\mathbf{B}}} n_{a, a^{\prime}} a z \otimes a^{\prime} z^{\prime} .
\end{aligned}
$$

We can now regard $M \otimes_{A} M^{\prime}$ as a $\dot{\mathbf{U}}_{A}$-module by restricting the $\hat{\mathbf{U}}_{A}^{(2)}$-module structure to $\dot{\mathbf{U}}_{A}$ via the algebra homomorphism $\Delta: \dot{\mathbf{U}}_{A} \rightarrow \hat{\mathbf{U}}_{A}^{(2)}$. Note that the resulting $\dot{\mathbf{U}}_{A}$-module $M \otimes_{A} M^{\prime}$ is the one defined in 1.6.

1.13. Let $\lambda, \lambda^{\prime}, \lambda_{1}, \lambda_{2}, \lambda_{1}^{\prime}, \lambda_{2}^{\prime}$ be elements of $X^{+}$such that $\lambda=\lambda_{1}+\lambda_{2}, \lambda^{\prime}=\lambda_{1}^{\prime}+\lambda_{2}^{\prime}$. Let $\mathfrak{T}: \Lambda_{\lambda_{1}^{\prime}+\lambda_{2}^{\prime}, \mathcal{A}} \rightarrow \Lambda_{\lambda_{1}^{\prime}, \mathcal{A}} \otimes_{\mathcal{A}} \Lambda_{\lambda_{2}^{\prime}, \mathcal{A}}$ be the restriction of the unique morphism $\Lambda_{\lambda_{1}^{\prime}+\lambda_{2}^{\prime}} \rightarrow \Lambda_{\lambda_{1}^{\prime}} \otimes \Lambda_{\lambda_{2}^{\prime}}$ in $\mathfrak{C}^{\prime}$ such that $\eta_{\lambda_{1}^{\prime}+\lambda_{2}^{\prime}} \mapsto \eta_{\lambda_{1}^{\prime}} \otimes \eta_{\lambda_{2}^{\prime}}$; see [L1, 25.1.2(a), 25.1.2(b)]. Similarly let $\mathfrak{T}^{\prime}:{ }^{\omega} \Lambda_{\lambda_{1}+\lambda_{2}, \mathcal{A}} \rightarrow{ }^{\omega} \Lambda_{\lambda_{1}, \mathcal{A}} \otimes{ }_{\mathcal{A}}{ }^{\omega} \Lambda_{\lambda_{2}, \mathcal{A}}$ be the restriction of the unique morphism ${ }^{\omega} \Lambda_{\lambda_{1}+\lambda_{2}} \rightarrow{ }^{\omega} \Lambda_{\lambda_{1}} \otimes{ }^{\omega} \Lambda_{\lambda_{2}}$ in $\mathfrak{C}$ such that $\xi_{-\lambda_{1}-\lambda_{2}} \mapsto \xi_{-\lambda_{1}} \otimes \xi_{-\lambda_{2}}$. Note that $\mathfrak{T}, \mathfrak{T}^{\prime}$ are morphisms in $\mathfrak{C}_{\mathcal{A}}$. We define

$$
\tau:{ }^{\omega} \Lambda_{\lambda, \mathcal{A}} \otimes_{\mathcal{A}} \Lambda_{\lambda^{\prime}, \mathcal{A}} \rightarrow{ }^{\omega} \Lambda_{\lambda_{1}, \mathcal{A}} \otimes_{\mathcal{A}} \Lambda_{\lambda_{1}^{\prime}, \mathcal{A}} \otimes_{\mathcal{A}}{ }^{\omega} \Lambda_{\lambda_{2}, \mathcal{A}} \otimes_{\mathcal{A}} \Lambda_{\lambda_{2}^{\prime}, \mathcal{A}}
$$

as the composition

$$
\begin{aligned}
& { }^{\omega} \Lambda_{\lambda, \mathcal{A}} \otimes_{\mathcal{A}} \Lambda_{\lambda^{\prime}, \mathcal{A}} \stackrel{\mathfrak{T}^{\prime} \otimes \mathfrak{T}}{\longrightarrow}{ }^{\omega} \Lambda_{\lambda_{1}, \mathcal{A}} \otimes_{\mathcal{A}}{ }^{\omega} \Lambda_{\lambda_{2}, \mathcal{A}} \otimes \Lambda_{\lambda_{1}^{\prime}, \mathcal{A}} \otimes_{\mathcal{A}} \Lambda_{\lambda_{2}^{\prime}, \mathcal{A}}
\end{aligned}
$$

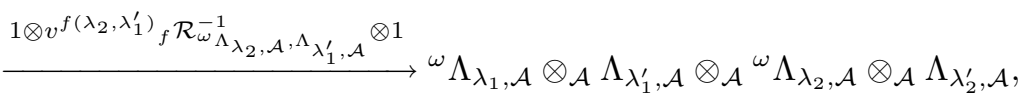

a morphism in $\mathfrak{C}_{\mathcal{A}}$. Note that $\tau$ depends on the choice of $f: X \times X \rightarrow \mathbf{Z}$ which is fixed as in 1.6. Define $\rho: \dot{\mathbf{U}}_{\mathcal{A}} \rightarrow{ }^{\omega} \Lambda_{\lambda, \mathcal{A}} \otimes_{\mathcal{A}} \Lambda_{\lambda^{\prime}, \mathcal{A}}$ by $u \mapsto u\left(\xi_{-\lambda} \otimes \eta_{\lambda^{\prime}}\right)$. Define

$$
\rho^{\prime}: \hat{\mathbf{U}}_{\mathcal{A}}^{(2)} \rightarrow{ }^{\omega} \Lambda_{\lambda_{1}, \mathcal{A}} \otimes_{\mathcal{A}} \Lambda_{\lambda_{1}^{\prime}, \mathcal{A}} \otimes_{\mathcal{A}}{ }^{\omega} \Lambda_{\lambda_{2}, \mathcal{A}} \otimes_{\mathcal{A}} \Lambda_{\lambda_{2}^{\prime}, \mathcal{A}}
$$

by $u \mapsto u\left(\xi_{-\lambda_{1}} \otimes_{\mathcal{A}} \eta_{\lambda_{1}^{\prime}} \otimes_{\mathcal{A}} \xi_{-\lambda_{2}} \otimes_{\mathcal{A}} \eta_{\lambda_{2}^{\prime}}\right)$. Here $M \otimes_{\mathcal{A}} M^{\prime}$ with $M={ }^{\omega} \Lambda_{\lambda_{1}, \mathcal{A}} \otimes_{\mathcal{A}} \Lambda_{\lambda_{1}^{\prime}, \mathcal{A}}$, $M^{\prime}={ }^{\omega} \Lambda_{\lambda_{2}, \mathcal{A}} \otimes_{\mathcal{A}} \Lambda_{\lambda_{2}^{\prime}, \mathcal{A}}$ is regarded as a $\hat{\mathbf{U}}_{\mathcal{A}}^{(2)}$-module as in 1.12. We show that the diagram

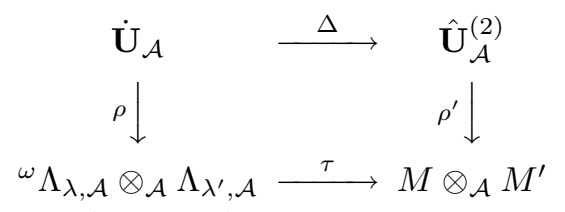

is commutative. We regard $\dot{\mathbf{U}}_{\mathcal{A}}$ as a $\dot{\mathbf{U}}_{\mathcal{A}}$-module via left multiplication. We regard $\hat{\mathbf{U}}_{\mathcal{A}}^{(2)}$ as a $\dot{\mathbf{U}}_{\mathcal{A}}$-module in which $u \in \dot{\mathbf{U}}_{\mathcal{A}}$ acts by left multiplication by $\Delta(u)$. Then $\Delta$ is $\dot{\mathbf{U}}_{\mathcal{A}}$-linear since $\Delta$ is an algebra homomorphism. From the definitions we see that $\rho$ and $\rho^{\prime}$ are $\dot{\mathbf{U}}_{\mathcal{A}}$-linear. Thus all maps in our diagram are $\dot{\mathbf{U}}_{\mathcal{A}}$-linear. Since $\left\{1_{x} ; x \in X\right\}$ generate the $\dot{\mathbf{U}}_{\mathcal{A}}$-module $\dot{\mathbf{U}}_{\mathcal{A}}$, it is enough to show that the two compositions in the diagram coincide when applied to any $1_{x}$ with $x \in X$. Thus it is enough to show that

$$
\tau\left(1_{x}\left(\xi_{-\lambda} \otimes \eta_{\lambda^{\prime}}\right)\right)=\sum_{x^{\prime}, x^{\prime \prime} \in X ; x^{\prime}+x^{\prime \prime}=x}\left(1_{x^{\prime}}\left(\xi_{-\lambda_{1}} \otimes \eta_{\lambda_{1}^{\prime}}\right)\right) \otimes\left(1_{x^{\prime \prime}}\left(\xi_{-\lambda_{2}} \otimes \eta_{\lambda_{2}^{\prime}}\right)\right)
$$

or equivalently that

$$
\tau\left(\xi_{-\lambda} \otimes \eta_{\lambda^{\prime}}\right)=\xi_{-\lambda_{1}} \otimes \eta_{\lambda_{1}^{\prime}} \otimes \xi_{-\lambda_{2}} \otimes \eta_{\lambda_{2}^{\prime}} .
$$

This follows from the definitions using the equality

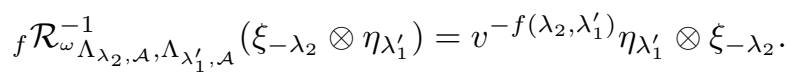


We now show:

(a) The $\mathcal{A}$-linear map $\tau$ is a split injection.

Since $\tau$ is the composition of $\mathfrak{T}^{\prime} \otimes_{\mathcal{A}} \mathfrak{T}$ with an $\mathcal{A}$-linear isomorphism, it is enough to show that $\mathfrak{T}^{\prime} \otimes_{\mathcal{A}} \mathfrak{T}$ is a split injection. It is also enough to show that the $\mathcal{A}$-linear maps $\mathfrak{T}$ and $\mathfrak{T}^{\prime}$ are split injections. By [L1, 27.1.7], $\mathfrak{T}$ carries $\underline{\mathbf{B}}_{\lambda^{\prime}}$ (an $\mathcal{A}$-basis of $\Lambda_{\lambda^{\prime}, \mathcal{A}}$ ) bijectively onto a subset of $\underline{\dot{\mathbf{B}}}_{\lambda_{1}^{\prime}, \lambda_{2}^{\prime}}\left(\right.$ an $\mathcal{A}$-basis of $\left.\Lambda_{\lambda_{1}^{\prime}, \mathcal{A}} \otimes_{\mathcal{A}} \Lambda_{\lambda_{2}^{\prime}, \mathcal{A}}\right)$. Hence $\mathfrak{T}$ is a split injection.

We identify the $\mathcal{A}$-modules ${ }^{\omega} \Lambda_{\lambda_{1}, \mathcal{A}} \otimes_{\mathcal{A}}{ }^{\omega} \Lambda_{\lambda_{2}, \mathcal{A}}$ and $\Lambda_{\lambda_{2}, \mathcal{A}} \otimes_{\mathcal{A}} \Lambda_{\lambda_{1}, \mathcal{A}}$ by $x \otimes y \leftrightarrow$ $y \otimes x$. Then $\mathfrak{T}^{\prime}$ becomes the $\mathcal{A}$-linear map $\Lambda_{\lambda, \mathcal{A}} \rightarrow \Lambda_{\lambda_{2}, \mathcal{A}} \otimes_{\mathcal{A}} \Lambda_{\lambda_{1}, \mathcal{A}}$ of the same type as $\mathfrak{T}$; hence it is a split injection by the previous argument for $\mathfrak{T}$. This proves $1.13(\mathrm{a})$.

1.14. Assume that $\lambda, \lambda^{\prime}, \lambda_{1}, \lambda_{2}, \lambda_{1}^{\prime}, \lambda_{2}^{\prime}$ in $X^{+}$are such that $\lambda=\lambda_{1}+\lambda_{2}, \lambda^{\prime}=\lambda_{1}^{\prime}+\lambda_{2}^{\prime}$. We show:

(a) If $a \in \dot{\mathbf{B}}_{\lambda_{1}, \lambda_{1}^{\prime}}, b \in \dot{\mathbf{B}}_{\lambda_{2}, \lambda_{2}^{\prime}}, c^{\prime} \in \dot{\mathbf{B}}-\dot{\mathbf{B}}_{\lambda, \lambda^{\prime}}$ then $\hat{m}_{c^{\prime}}^{a, b}=0$ in $\mathcal{A}$.

In the commutative diagram in 1.13 we have $\rho\left(c^{\prime}\right)=0$, hence $\rho^{\prime}\left(\Delta\left(c^{\prime}\right)\right)=0$, that is, $\rho^{\prime}\left(\sum_{a, b \in \dot{\mathbf{B}}} \hat{m}_{c^{\prime}}^{a, b} a \otimes b\right)=0$ so that

$$
\sum_{a, b \in \dot{\mathbf{B}}} \hat{m}_{c^{\prime}}^{a, b}\left(a\left(\xi_{-\lambda_{1}} \otimes \eta_{\lambda_{1}^{\prime}}\right) \otimes b\left(\xi_{-\lambda_{2}} \otimes \eta_{\lambda_{2}^{\prime}}\right)\right)=0 .
$$

The term corresponding to $(a, b)$ is 0 unless $(a, b) \in \dot{\mathbf{B}}_{\lambda_{1}, \lambda_{1}^{\prime}} \times \dot{\mathbf{B}}_{\lambda_{2}, \lambda_{2}^{\prime}}$; moreover when $(a, b)$ runs through $\dot{\mathbf{B}}_{\lambda_{1}, \lambda_{1}^{\prime}} \times \dot{\mathbf{B}}_{\lambda_{2}, \lambda_{2}^{\prime}}$, the elements $a\left(\xi_{-\lambda_{1}} \otimes \eta_{\lambda_{1}^{\prime}}\right) \otimes b\left(\xi_{-\lambda_{2}} \otimes \eta_{\lambda_{2}^{\prime}}\right)$ are linearly independent (they form the set $\underline{\dot{\mathbf{B}}}_{\lambda_{1}, \lambda_{1}^{\prime}} \otimes \underline{\mathbf{\mathbf { B }}}_{\lambda_{2}, \lambda_{2}^{\prime}}$ ). It follows that $\hat{m}_{c^{\prime}}^{a, b}=0$ for any $(a, b) \in \dot{\mathbf{B}}_{\lambda_{1}, \lambda_{1}^{\prime}} \times \dot{\mathbf{B}}_{\lambda_{2}, \lambda_{2}^{\prime}}$. This proves 1.14(a).

1.15. In the setup of 1.14 , we show:

(a) Let $c \in \dot{\mathbf{B}}_{\lambda, \lambda^{\prime}}$. There exists a function $h: \dot{\mathbf{B}}_{\lambda_{1}, \lambda_{1}^{\prime}} \times \dot{\mathbf{B}}_{\lambda_{2}, \lambda_{2}^{\prime}} \rightarrow \mathcal{A}$ such that for any $c^{\prime} \in \dot{\mathbf{B}}$ we have

$$
\sum_{a \in \dot{\mathbf{B}}_{\lambda_{1}, \lambda_{1}^{\prime}}, b \in \dot{\mathbf{B}}_{\lambda_{2}, \lambda_{2}^{\prime}}} h(a, b) \hat{m}_{c^{\prime}}^{a, b}=\delta_{c, c^{\prime}}
$$

For any $\tilde{\lambda}, \tilde{\lambda}^{\prime}$ in $X^{+}$, we set $b^{\dagger}=b\left(\xi_{-\tilde{\lambda}} \otimes_{\mathcal{A}} \eta_{\tilde{\lambda}^{\prime}}\right)$ for $b \in \dot{\mathbf{B}}_{\tilde{\lambda}, \tilde{\lambda}^{\prime}}$ so that $\left\{b^{\dagger} ; b \in \dot{\mathbf{B}}_{\tilde{\lambda}, \tilde{\lambda}^{\prime}}\right\}$ is an $\mathcal{A}$-basis of ${ }^{\omega} \Lambda_{\tilde{\lambda}, \mathcal{A}} \otimes_{\mathcal{A}} \Lambda_{\tilde{\lambda}^{\prime}, \mathcal{A}}$ and we denote by $\left\{\hat{b} ; b \in \dot{\mathbf{B}}_{\tilde{\lambda}, \tilde{\lambda}^{\prime}}\right\}$ the dual $\mathcal{A}$-basis of $\operatorname{Hom}_{\mathcal{A}}\left({ }^{\omega} \Lambda_{\tilde{\lambda}, \mathcal{A}} \otimes_{\mathcal{A}} \Lambda_{\tilde{\lambda}^{\prime}, \mathcal{A}}, \mathcal{A}\right)$. In particular the bases $\left\{\hat{c}^{\prime} ; c^{\prime} \in \dot{\mathbf{B}}_{\lambda, \lambda^{\prime}}\right\},\left\{\hat{a}^{\prime} ; a^{\prime} \in\right.$ $\left.\dot{\mathbf{B}}_{\lambda_{1}, \lambda_{1}^{\prime}}\right\},\left\{\hat{b}^{\prime} ; b^{\prime} \in \dot{\mathbf{B}}_{\lambda_{2}, \lambda_{2}^{\prime}}\right\}$ of $\operatorname{Hom}_{\mathcal{A}}\left(\Lambda_{\lambda, \mathcal{A}} \otimes_{\mathcal{A}} \Lambda_{\lambda^{\prime}, \mathcal{A}}, \mathcal{A}\right), \operatorname{Hom}_{\mathcal{A}}\left(\Lambda_{\lambda_{1}, \mathcal{A}} \otimes_{\mathcal{A}} \Lambda_{\lambda_{1}^{\prime}, \mathcal{A}}, \mathcal{A}\right)$, $\operatorname{Hom}_{\mathcal{A}}\left(\Lambda_{\lambda_{2}, \mathcal{A}} \otimes_{\mathcal{A}} \Lambda_{\lambda_{2}^{\prime}, \mathcal{A}}, \mathcal{A}\right)$ are defined. By 1.13(a), we can find an $\mathcal{A}$-linear map

$$
\psi: \Lambda_{\lambda_{1}, \mathcal{A}} \otimes_{\mathcal{A}} \Lambda_{\lambda_{1}^{\prime}, \mathcal{A}} \otimes_{\mathcal{A}} \Lambda_{\lambda_{2}, \mathcal{A}} \otimes_{\mathcal{A}} \Lambda_{\lambda_{2}^{\prime}, \mathcal{A}} \rightarrow \Lambda_{\lambda, \mathcal{A}} \otimes_{\mathcal{A}} \Lambda_{\lambda^{\prime}, \mathcal{A}}
$$

such that $\psi \tau=1$ with $\tau$ as in 1.13 . The transpose maps ${ }^{t} \psi,{ }^{t} \tau$ satisfy ${ }^{t} \tau\left({ }^{t} \psi\right)=1$. We can write uniquely

$$
{ }^{t} \psi(\hat{c})=\sum_{a^{\prime} \in \dot{\mathbf{B}}_{\lambda_{1}, \lambda_{1}^{\prime}}, b^{\prime} \in \dot{\mathbf{B}}_{\lambda_{2}, \lambda_{2}^{\prime}}} h\left(a^{\prime}, b^{\prime}\right) \hat{a}^{\prime} \otimes \hat{b}^{\prime}
$$

with $h\left(a^{\prime}, b^{\prime}\right) \in \mathcal{A}$. Hence

$$
\hat{c}=\sum_{a^{\prime} \in \dot{\mathbf{B}}_{\lambda_{1}, \lambda_{1}^{\prime}}, b^{\prime} \in \dot{\mathbf{B}}_{\lambda_{2}, \lambda_{2}^{\prime}}} h\left(a^{\prime}, b^{\prime}\right)^{t} \tau\left(\hat{a}^{\prime} \otimes \hat{b}^{\prime}\right) .
$$


Evaluating both sides at $c^{\prime \dagger}$ with $c^{\prime} \in \dot{\mathbf{B}}_{\lambda, \lambda^{\prime}}$ and using

$$
\tau\left(c^{\prime \dagger}\right)=\sum_{a \in \dot{\mathbf{B}}_{\lambda_{1}, \lambda_{1}^{\prime}}, b \in \dot{\mathbf{B}}_{\lambda_{2}, \lambda_{2}^{\prime}}} \hat{m}_{c^{\prime}}^{a, b} a^{\dagger} \otimes b^{\dagger}
$$

(which follows from the commutative diagram in 1.13), we have

$$
\delta_{c, c^{\prime}}=\sum_{a, a^{\prime} \in \dot{\mathbf{B}}_{\lambda_{1}, \lambda_{1}^{\prime}}, b, b^{\prime} \in \dot{\mathbf{B}}_{\lambda_{2}, \lambda_{2}^{\prime}}} h\left(a^{\prime}, b^{\prime}\right) \hat{m}_{c^{\prime}}^{a, b} \delta_{a^{\prime}, a} \delta_{b^{\prime}, b},
$$

that is,

$$
\delta_{c, c^{\prime}}=\sum_{a \in \dot{\mathbf{B}}_{\lambda_{1}, \lambda_{1}^{\prime}}, b \in \dot{\mathbf{B}}_{\lambda_{2}, \lambda_{2}^{\prime}}} h(a, b) \hat{m}_{c^{\prime}}^{a, b} .
$$

This also holds when $c^{\prime} \in \dot{\mathbf{B}}-\dot{\mathbf{B}}_{\lambda, \lambda^{\prime}}$ (both sides are 0 by 1.14(a)). This proves $1.15(\mathrm{a})$.

Lemma 1.16. For any $a, b$ in $\dot{\mathbf{B}}$, the set $\left\{c \in \dot{\mathbf{B}} ; \hat{m}_{c}^{a, b} \in \mathcal{A}-\{0\}\right\}$ is finite.

We have $a \in \dot{\mathbf{B}}_{\lambda_{1}, \lambda_{1}^{\prime}}, b \in \dot{\mathbf{B}}_{\lambda_{2}, \lambda_{2}^{\prime}}$ for some $\lambda_{1}, \lambda_{1}^{\prime}, \lambda_{2}, \lambda_{2}^{\prime}$ in $X^{+}$. By 1.14(a) we have $\left\{c \in \dot{\mathbf{B}} ; \hat{m}_{c}^{a, b} \in \mathcal{A}-\{0\}\right\} \subset \dot{\mathbf{B}}_{\lambda_{1}+\lambda_{2}, \lambda_{1}^{\prime}+\lambda_{2}^{\prime}}$. Since $\dot{\mathbf{B}}_{\lambda_{1}+\lambda_{2}, \lambda_{1}^{\prime}+\lambda_{2}^{\prime}}$ is finite, the lemma holds.

1.17. We define an $A$-linear map $\hat{D}: \hat{\mathbf{U}}_{A} \rightarrow \hat{\mathbf{U}}_{A}^{(2)}$ by

$$
\sum_{c \in \dot{\mathbf{B}}} n_{c} c \mapsto \sum_{(a, b) \in \dot{\mathbf{B}} \times \dot{\mathbf{B}}}\left(\sum_{c \in \dot{\mathbf{B}}} \hat{m}_{c}^{a, b} n_{c}\right) a \otimes b .
$$

This make sense: for any $(a, b) \in \dot{\mathbf{B}} \times \dot{\mathbf{B}}$, the sum $\sum_{c \in \dot{\mathbf{B}}} \hat{m}_{c}^{a, b} n_{c}$ has only finitely many non-zero terms. (See 1.16.) Using 1.5(iii), we see that $\hat{D}$ is an $A$-algebra homomorphism. It clearly extends the homomorphism $\Delta: \dot{\mathbf{U}}_{A} \rightarrow \hat{\mathbf{U}}_{A}^{(2)}$. From $1.5($ vi), (viii) we see that

$$
\hat{D}(S(\xi))=S^{(2)} \operatorname{tr} \hat{D}(\xi), \quad \hat{D}(\omega(\xi))=\omega^{(2)} \operatorname{tr} \hat{D}(\xi)
$$

for any $\xi \in \hat{\mathbf{U}}_{A}$ where $S^{(2)}, \omega^{(2)}$ are as in 1.11 and $\operatorname{tr}: \hat{\mathbf{U}}_{A}^{(2)} \rightarrow \hat{\mathbf{U}}_{A}^{(2)}$ is the $A$-linear map given by

$$
\sum_{\left(a, a^{\prime}\right) \in \dot{\mathbf{B}} \times \dot{\mathbf{B}}} n_{a, a^{\prime}} a \otimes a^{\prime} \mapsto \sum_{\left(a, a^{\prime}\right) \in \dot{\mathbf{B}} \times \dot{\mathbf{B}}} n_{a^{\prime}, a} a \otimes a^{\prime} .
$$

If $v=1$ in $A$, then $\operatorname{tr} \hat{D}(\xi)=\hat{D}(\xi)$ for $\xi \in \hat{\mathbf{U}}_{A}$ (see $1.5($ xiii)). Hence in this case we have $\hat{D}(S(\xi))=S^{(2)} \hat{D}(\xi), \hat{D}(\omega(\xi))=\omega^{(2)} \hat{D}(\xi)$ for $\xi \in \hat{\mathbf{U}}_{A}$.

1.18. For any $i \in I$ and $h \in A$ we set

$$
\begin{aligned}
& x_{i}(h)=\sum_{c \in \mathbf{N}, \lambda \in X} h^{c} \theta_{i}^{(c)+} 1_{\lambda} \in \hat{\mathbf{U}}_{A}, \\
& y_{i}(h)=\sum_{c \in \mathbf{N}, \lambda \in X} h^{c} \theta_{i}^{(c)-} 1_{\lambda} \in \hat{\mathbf{U}}_{A} .
\end{aligned}
$$

Note that $\theta_{i}^{(t) \pm} 1_{\lambda} \in \dot{\mathbf{B}}$; see [L1, 25.3.1]. We have

$$
y_{i}(h)=\omega\left(x_{i}(h)\right) .
$$


We show:

(a) If $v=1$ in $A$, then $\hat{D}\left(x_{i}(h)\right)=x_{i}(h) \otimes x_{i}(h) \in \hat{\mathbf{U}}_{A}^{(2)}, \hat{D}\left(y_{i}(h)\right)=y_{i}(h) \otimes$ $y_{i}(h) \in \hat{\mathbf{U}}_{A}^{(2)}$.

Without assumption on $v \in A$ we have (using [L1, 3.1.5]):

$$
\begin{aligned}
& \hat{D}\left(x_{i}(h)\right)=\sum_{t^{\prime}, t^{\prime \prime} \in \mathbf{N}, \lambda^{\prime}, \lambda^{\prime \prime} \in X} h^{t^{\prime}+t^{\prime \prime}} v_{i}^{t^{\prime} t^{\prime \prime}} v_{i}^{\left\langle i, \lambda^{\prime}\right\rangle t^{\prime \prime}}\left(\theta_{i}^{\left(t^{\prime}\right)+} 1_{\lambda^{\prime}}\right) \otimes\left(\theta_{i}^{\left(t^{\prime \prime}\right)+} 1_{\lambda^{\prime \prime}}\right), \\
& \hat{D}\left(y_{i}(h)\right)=\sum_{t^{\prime}, t^{\prime \prime} \in \mathbf{N}, \lambda^{\prime}, \lambda^{\prime \prime} \in X} h^{t^{\prime}+t^{\prime \prime}} v_{i}^{-t^{\prime} t^{\prime \prime}} v_{i}^{-\left\langle i, \lambda^{\prime \prime}\right\rangle t^{\prime}}\left(\theta_{i}^{\left(t^{\prime}\right)-} 1_{\lambda^{\prime}}\right) \otimes\left(\theta_{i}^{\left(t^{\prime \prime}\right)-} 1_{\lambda^{\prime \prime}}\right) .
\end{aligned}
$$

The desired formulas follow.

We show:

(b) If $v=1$ in $A$ and $h, h^{\prime} \in A$, then $x_{i}\left(h+h^{\prime}\right)=x_{i}(h) x_{i}\left(h^{\prime}\right) \in \hat{\mathbf{U}}_{A}, y_{i}\left(h+h^{\prime}\right)=$ $y_{i}(h) y_{i}\left(h^{\prime}\right) \in \hat{\mathbf{U}}_{A}$.

Without assumption on $v \in A$ we have

$$
\begin{aligned}
& x_{i}(h) x_{i}\left(h^{\prime}\right)=\sum_{t \in \mathbf{N}, \lambda \in X}\left(\sum_{t^{\prime}, t^{\prime \prime} ; t^{\prime}+t^{\prime \prime}=t} h^{t^{\prime}} h^{\prime t^{\prime \prime}}\left[\begin{array}{c}
t \\
t^{\prime}
\end{array}\right]_{i}\right) \theta_{i}^{(t)+} 1_{\lambda}, \\
& y_{i}(h) y_{i}\left(h^{\prime}\right)=\sum_{t \in \mathbf{N}, \lambda \in X}\left(\sum_{t^{\prime}, t^{\prime \prime} ; t^{\prime}+t^{\prime \prime}=t} h^{t^{\prime}} h^{\prime t^{\prime \prime}}\left[\begin{array}{c}
t \\
t^{\prime}
\end{array}\right]_{i}\right) \theta_{i}^{(t)-} 1_{\lambda} .
\end{aligned}
$$

If $v=1$, we have $\left[\begin{array}{c}t \\ t^{\prime}\end{array}\right]_{i}=\left(\begin{array}{c}t \\ t^{\prime}\end{array}\right)$ and $\sum_{t^{\prime}, t^{\prime \prime} ; t^{\prime}+t^{\prime \prime}=t} h^{t^{\prime}} h^{\prime t^{\prime \prime}}\left[\begin{array}{c}t \\ t^{\prime}\end{array}\right]_{i}=\left(h+h^{\prime}\right)^{t}$. The desired formulas follow.

1.19. We say that a (possibly infinite) $\operatorname{sum} \sum_{f \in F} p_{f}$ with $p_{f} \in \hat{\mathbf{U}}_{A}$ is defined in $\hat{\mathbf{U}}_{A}$ if, when setting $p_{f}=\sum_{a \in \dot{\mathbf{B}}} n_{a, f} a \in \hat{\mathbf{U}}_{A}\left(n_{a, f} \in A\right)$, the set $\left\{f \in F ; n_{a, f} \neq 0\right\}$ is finite for any $a \in \dot{\mathbf{B}}$. In this case we assign to the sum the value $\sum_{a \in \dot{\mathbf{B}}}\left(\sum_{f \in F} n_{a, f}\right) a \in$ $\hat{\mathbf{U}}_{A}$. Using 1.8 we see that, if the (possibly infinite) sums $\sum_{f \in F} p_{f}, \sum_{f^{\prime} \in F^{\prime}} p_{f^{\prime}}^{\prime}$ are defined in $\hat{\mathbf{U}}_{A}$, then the sum $\sum_{\left(f, f^{\prime}\right) \in F \times F^{\prime}} p_{f} p_{f^{\prime}}^{\prime}$ is defined in $\hat{\mathbf{U}}_{A}$ and we have

$$
\left(\sum_{f \in F} p_{f}\right)\left(\sum_{f^{\prime} \in F^{\prime}} p_{f^{\prime}}^{\prime}\right)=\sum_{\left(f, f^{\prime}\right) \in F \times F^{\prime}} p_{f} p_{f^{\prime}}^{\prime}, \text { in } \hat{\mathbf{U}}_{A} \text {. }
$$

2. The ELEMENTS $s_{i, e}^{\prime}, s_{i, e}^{\prime \prime}$ OF $\widehat{\mathbf{U}}_{A}$

2.1. In this section we introduce and study some elements $s_{i, e}^{\prime}, s_{i, e}^{\prime \prime}$ of $\hat{\mathbf{U}}_{A}$ which implement the braid group action [L1, Ch.39] on $\dot{\mathbf{U}}_{A}$.

Let $i \in I, e= \pm 1$. Let

$$
\begin{aligned}
& s_{i, e}^{\prime}=\sum_{y \in \mathbf{Z}, x \in \mathbf{Z}, \lambda \in X ;\langle i, \lambda\rangle=x+y}(-1)^{y} v_{i}^{e y} \theta_{i}^{(x)-} 1_{\lambda} \theta_{i}^{(y)+} \in \hat{\mathbf{U}}_{A}, \\
& s_{i, e}^{\prime \prime}=\sum_{y \in \mathbf{Z}, x \in \mathbf{Z}, \lambda \in X ;\langle i, \lambda\rangle=x+y}(-1)^{x} v_{i}^{e x} \theta_{i}^{(x)-} 1_{\lambda} \theta_{i}^{(y)+} \in \hat{\mathbf{U}}_{A} .
\end{aligned}
$$

Note that in the formulas above each $\theta_{i}^{(x)-} 1_{\lambda} \theta_{i}^{(y)+}$ belongs to $\dot{\mathbf{B}}$ (see [L1, 25.3.1]). We have $\omega\left(s_{i, e}^{\prime}\right)=s_{i, e}^{\prime \prime}$. Indeed, using the definition of $\omega$ (see 1.4), we have

$$
\omega\left(\theta_{i}^{(x)-} 1_{\lambda} \theta_{i}^{(y)+}\right)=\theta_{i}^{(x)+} 1_{-\lambda} \theta_{i}^{(y)-}=\theta_{i}^{(y)-} 1_{\lambda} \theta_{i}^{(x)+}
$$

for any $y \in \mathbf{Z}, x \in \mathbf{Z}, \lambda \in X$ such that $\langle i, \lambda\rangle=x+y$. 
Lemma 2.2. We preserve the setup of 2.1. For any $r \in \mathbf{Z}$ we consider the sums

$$
\begin{gathered}
\sum_{m, n, p \in \mathbf{N}, \lambda \in X ;\langle i, \lambda\rangle=m-n+p-r}(-1)^{n} v_{i}^{e(-m p+n)} \theta_{i}^{(m)-} \theta_{i}^{(n)+} \theta_{i}^{(p)-} 1_{\lambda}, \\
\sum_{m, n, p \in \mathbf{N}, \lambda \in X ;\langle i, \lambda\rangle=-m+n-p+r}(-1)^{n} v_{i}^{e(-m p+n)} \theta_{i}^{(m)+} \theta_{i}^{(n)-} \theta_{i}^{(p)+} 1_{\lambda} .
\end{gathered}
$$

(a) The sums (i), (ii) are defined in $\hat{\mathbf{U}}_{A}$. Let $\tau_{i, e, r}^{\prime}, \tau_{i, e, r}^{\prime \prime}$ be their value in $\hat{\mathbf{U}}_{A}$.

(b) We have $\tau_{i, e, 0}^{\prime}=s_{i, e}^{\prime}, \tau_{i, e, 0}^{\prime \prime}=s_{i, e}^{\prime \prime}$.

(c) If $v=1$ in $A$ and $r \in \mathbf{Z}-\{0\}$, we have $\tau_{i, e, r}^{\prime}=0, \tau_{i, e, r}^{\prime \prime}=0$.

(d) If $v=1$ in $A$, we have $s_{i, e}^{\prime \prime}=x_{i}(1) y_{i}(-1) x_{i}(1), s_{i, e}^{\prime}=y_{i}(1) x_{i}(-1) y_{i}(1)$ in $\hat{\mathbf{U}}_{A}$.

(e) If $v=1$ in $A$, we have $\hat{D}\left(s_{i, e}^{\prime}\right)=s_{i, e}^{\prime} \otimes s_{i, e}^{\prime}$ and $\hat{D}\left(s_{i, e}^{\prime \prime}\right)=s_{i, e}^{\prime \prime} \otimes s_{i, e}^{\prime \prime}$ in $\hat{\mathbf{U}}_{A}^{(2)}$.

We compute formally the sum (ii) for $r \in \mathbf{N}$ (using 1.4):

$$
\begin{aligned}
& \sum_{m, n, p \in \mathbf{N}, \lambda \in X ;\langle i, \lambda\rangle=-m+n-p+r}(-1)^{n} v_{i}^{e(-m p+n)} \theta_{i}^{(m)+} 1_{\lambda+p i^{\prime}-n i^{\prime}} \theta_{i}^{(n)-} \theta_{i}^{(p)+} \\
= & \sum_{m \in \mathbf{Z}, n \in \mathbf{Z}, p \in \mathbf{N}, t \in \mathbf{N}, \lambda \in X ;\langle i, \lambda\rangle=-m+n-p+r}(-1)^{n} v_{i}^{e(-m p+n)} \\
& \left.\times \begin{array}{c}
m+n-\left\langle i,-\lambda-p i^{\prime}+n i^{\prime}\right\rangle \\
t
\end{array}\right]_{i} \theta_{i}^{(n-t)-} 1_{\lambda+p i^{\prime}-n i^{\prime}+(n+m-t) i^{\prime}} \theta_{i}^{(m-t)+} \theta_{i}^{(p)+} \\
= & \sum_{m \in \mathbf{Z}, n \in \mathbf{Z}, p \in \mathbf{N}, t \in \mathbf{N}, \lambda \in X ;\langle i, \lambda\rangle=-m+n-p+r}(-1)^{n} v_{i}^{e(-m p+n)}\left[\begin{array}{c}
r+p \\
t
\end{array}\right]_{i} \\
= & \left.\sum_{m \in \mathbf{Z}, x \in \mathbf{Z}, p \in \mathbf{N}, t \in \mathbf{N}, \lambda \in X ;\langle i, \lambda\rangle=-m+x+t-p+r}^{m+p-t}\right]_{i} \theta_{i}^{(n-t)-} 1_{\lambda+p i^{\prime}-n i^{\prime}+(n+m-t) i^{\prime}} \theta_{i}^{(m+p-t)+} \\
& \times\left[\begin{array}{c}
m+p-t)^{x+t} v_{i}^{e(-m p+x+t)}\left[\begin{array}{c}
r+p \\
t
\end{array}\right]_{i} \\
p
\end{array}\right]_{i} \theta_{i}^{(x)-} 1_{\lambda+(m+p-t) i^{\prime}} \theta_{i}^{(m+p-t)+} \\
= & \sum_{y \in \mathbf{N}, x \in \mathbf{N}, \lambda \in X ;}(-1)^{x+t} v_{i}^{e(-(y-p+t) p+x+t)}\left[\begin{array}{c}
r+p \\
t
\end{array}\right]_{i}\left[\begin{array}{c}
y \\
p
\end{array}\right]_{i} c_{x, y, \lambda ; r} \theta_{i}^{(x)-} 1_{\lambda+y i^{\prime}} \theta_{i}^{(y)+}
\end{aligned}
$$

where each $\theta_{i}^{(x)-} 1_{\lambda+y i^{\prime}} \theta_{i}^{(y)+}$ belongs to $\dot{\mathbf{B}}$ (see [L1, 25.3.1]) and

$$
c_{x, y, \lambda ; r}=\sum_{p \in \mathbf{N}, t \in \mathbf{N}}(-1)^{x+t} v_{i}^{e(-(y-p+t) p+x+t)}\left[\begin{array}{c}
r+p \\
t
\end{array}\right]_{i}\left[\begin{array}{l}
y \\
p
\end{array}\right]_{i} .
$$

Note that only finitely many terms of this sum can $b e \neq 0$ (those for which $p \leq y$ and $t \leq r+p$ ); here we have used that $r \in \mathbf{N}$. In particular the sum (ii) is defined in $\hat{\mathbf{U}}_{A}$ and has a value $\tau_{i, e ; r}^{\prime \prime}$ in $\hat{\mathbf{U}}_{A}$. 
Next we compute formally the sum (ii) for $r \in-\mathbf{N}$ (using 1.4):

$$
\begin{aligned}
& \sum_{m, n, p \in \mathbf{N}, \lambda \in X ;\langle i, \lambda\rangle=-m+n-p+r}(-1)^{n} v_{i}^{e(-m p+n)} \theta_{i}^{(m)+} \theta_{i}^{(n)-} 1_{\lambda+p i^{\prime}} \theta_{i}^{(p)+} \\
= & \sum_{m \in \mathbf{N}, n \in \mathbf{Z}, p \in \mathbf{Z}, t \in \mathbf{N}, \lambda \in X ;\langle i, \lambda\rangle=-m+n-p+r}(-1)^{n} v_{i}^{e(-m p+n)}\left[\begin{array}{c}
n+p-\left\langle i, \lambda+p i^{\prime}\right\rangle \\
t
\end{array}\right]_{i} \\
& \times \theta_{i}^{(m)+} \theta_{i}^{(p-t)+} 1_{\lambda+p i^{\prime}-(n+p-t) i^{\prime}} \theta_{i}^{(n-t)-} \\
= & \sum_{m \in \mathbf{N}, n \in \mathbf{Z}, p \in \mathbf{Z}, t \in \mathbf{N}, \lambda \in X ;\langle i, \lambda\rangle=-m+n-p+r}(-1)^{n} v_{i}^{e(-m p+n)}\left[\begin{array}{c}
m-r \\
t
\end{array}\right]_{i} \\
= & \left.\times \begin{array}{c}
m+p-t \\
m
\end{array}\right]_{i} \theta_{i}^{(m+p-t)+} 1_{\lambda-(n-t) i^{\prime}} \theta_{i}^{(n-t)-} \\
& \sum_{m \in \mathbf{N}, x \in \mathbf{Z}, y \in \mathbf{Z}, t \in \mathbf{N}, \lambda \in X ;\langle i, \lambda\rangle=y-x+r}(-1)^{x+t} v_{i}^{e(-m(x+t-m)+y+t)}\left[\begin{array}{c}
m-r \\
t
\end{array}\right]_{i} \\
& \times\left[\begin{array}{c}
x \\
m
\end{array}\right]_{i} \theta_{i}^{(x)+}{ }_{1_{\lambda-y i^{\prime}} \theta_{i}^{(y)-}} \sum_{x \in \mathbf{N}, y \in \mathbf{N}, \lambda \in X ;\langle i, \lambda\rangle=y-x+r} \tilde{c}_{x, y, \lambda ; r} \theta_{i}^{(x)+} 1_{\lambda-y i^{\prime}} \theta_{i}^{(y)-},
\end{aligned}
$$

where the last product $\theta_{i}^{(x)+} 1_{\lambda-y i^{\prime}} \theta_{i}^{(y)-}$ belongs to $\dot{\mathbf{B}}$ (see [L1, 25.3.1]) and

$$
\tilde{c}_{x, y, \lambda ; r}=\sum_{m \in \mathbf{N}, t \in \mathbf{N}}(-1)^{x+t} v_{i}^{e(-m(x+t-m)+y+t)}\left[\begin{array}{c}
m-r \\
t
\end{array}\right]_{i}\left[\begin{array}{c}
x \\
m
\end{array}\right]_{i} .
$$

Note that only finitely many terms of this sum can $b e \neq 0$ (those for which $m \leq x$ and $t \leq m-r$ ); here we have used that $r \in-\mathbf{N}$. In particular the sum (ii) is defined in $\hat{\mathbf{U}}_{A}$ and has a value $\tau_{i, e ; r}^{\prime \prime}$ in $\hat{\mathbf{U}}_{A}$.

From the computations above we see also that the sum

$$
\sum_{m, n, p \in \mathbf{N}, \lambda \in X}(-1)^{n} v_{i}^{e(-m p+n)} \theta_{i}^{(m)+} \theta_{i}^{(n)-} \theta_{i}^{(p)+} 1_{\lambda}
$$

is defined in $\hat{\mathbf{U}}_{A}$ and its value $\tau_{i, e}^{\prime \prime} \in \hat{\mathbf{U}}_{A}$ is equal to $\sum_{r \in \mathbf{Z}} \tau_{i, e, r}^{\prime \prime}$.

In the formula for $c_{x, y, \lambda ; 0}$ we have

$$
\sum_{t \in \mathbf{N}}(-1)^{x+t} v_{i}^{e(-(y-p+t) p+x+t)}\left[\begin{array}{c}
r+p \\
t
\end{array}\right]_{i}=(-1)^{x} v_{i}^{e(-(y-p) p+x)} \delta_{p, 0}=(-1)^{x} v_{i}^{e x} \delta_{p, 0},
$$

hence $c_{x, y, \lambda ; 0}=(-1)^{x} v_{i}^{e x}$ and

$$
\tau_{i, e ; 0}^{\prime \prime}=\sum_{y \in \mathbf{Z}, x \in \mathbf{Z}, \lambda \in X ;\langle i, \lambda\rangle=-y+x}(-1)^{x} v_{i}^{e x} \theta_{i}^{(x)-} 1_{\lambda+y i^{\prime}} \theta_{i}^{(y)+} .
$$

We see that $\tau_{i, e ; 0}^{\prime \prime}=s_{i, e}^{\prime \prime}$. Similarly, we see that the family defining the sum (i) is defined, hence it has a value $\tau_{i, e ; r}^{\prime}$ in $\hat{\mathbf{U}}_{A}$, that the sum

$$
\sum_{m, n, p \in \mathbf{N}, \lambda \in X}(-1)^{n} v_{i}^{e(-m p+n)} \theta_{i}^{(m)-} \theta_{i}^{(n)+} \theta_{i}^{(p)-} 1_{\lambda}
$$


is defined in $\hat{\mathbf{U}}_{A}$ and its value $\tau_{i, e}^{\prime} \in \hat{\mathbf{U}}_{A}$ is equal to $\sum_{r \in \mathbf{Z}} \tau_{i, e, r}^{\prime}$. We see also that $\tau_{i, e ; 0}^{\prime}=s_{i, e}^{\prime}$. (These statements could also be deduced from the earlier part of the proof using that $\tau_{i, e ; r}^{\prime \prime}=\omega\left(\tau_{i, e . r}^{\prime}\right)$.)

In the remainder of the proof we assume that $v=1$ in $A$. In this case in the formula defining $c_{x, y, \lambda ; r}$ with $r>0$ we have $\sum_{t \in \mathbf{N}}(-1)^{x+t}\left[\begin{array}{c}r+p \\ t\end{array}\right]_{i}=0$ since $r+p>0$ and $v=1$. Hence $c_{x, y, \lambda ; r}=0$ and $\tau_{i, e ; r}^{\prime \prime}=0$ for $r>0$. In the formula defining $\tilde{c}_{x, y, \lambda ; r}$ with $r<0$ we have $\sum_{t \in \mathbf{N}}(-1)^{x+t}\left[\begin{array}{c}m-r \\ t\end{array}\right]_{i}=0$ since $m-r>0$, hence $\tilde{c}_{x, y, \lambda ; r}=0$ and $\tau_{i, e ; r}^{\prime \prime}=0$ for $r<0$. We see that $\tau_{i, e ; r}^{\prime \prime}=0$ for $r \neq 0$ and $\tau_{i, e}^{\prime \prime}=\tau_{i, e ; 0}^{\prime \prime}=s_{i, e}^{\prime \prime}$. Applying $\omega$, we see that $\tau_{i, e ; r}^{\prime}=0$ for $r \neq 0$ and $\tau_{i, e}^{\prime}=\tau_{i, e ; 0}^{\prime}=s_{i, e}^{\prime}$. It is clear that $\tau_{i, e}^{\prime \prime}=x_{i}(1) y_{i}(-1) x_{i}(1), \tau_{i, e}^{\prime}=y_{i}(1) x_{i}(-1) y_{i}(1)$ in $\hat{\mathbf{U}}_{A}$. Hence (d) follows. Now (e) follows from (d) using 1.18(a) and the fact that $\hat{D}: \hat{\mathbf{U}}_{A} \rightarrow \hat{\mathbf{U}}_{A}^{(2)}$ is an algebra homomorphism.

2.3. Let $i \in I, e= \pm 1$. Let $T_{i, e}^{\prime}, T_{i, e}^{\prime \prime}$ be the algebra automorphisms of $\dot{\mathbf{U}}_{A}$ defined in [L1, 41.1.8]. For any $M \in \mathfrak{C}_{A}$ let $T_{i, e}^{\prime}: M \rightarrow M, T_{i, e}^{\prime \prime}: M \rightarrow M$ be the $A$-linear isomorphisms defined in [L1, 41.2.3]. From the definitions we have $T_{i, e}^{\prime}(z)=\tau_{i, e ; 0}^{\prime} z$, $T_{i, e}^{\prime \prime}(z)=\tau_{i, e ; 0}^{\prime \prime} z$ for $z \in M$ (using the $\hat{\mathbf{U}}_{A^{-}}$-module structure of $M$ ); here we use notation of Lemma 2.2(a). By [L1, 41.2.4], for $u \in \dot{\mathbf{U}}_{A}, z \in M$ we have

$$
T_{i, e}^{\prime}(u z)=T_{i, e}^{\prime}(u)\left(T_{i, e}^{\prime}(z)\right), \quad T_{i, e}^{\prime \prime}(u z)=T_{i, e}^{\prime \prime}(u)\left(T_{i, e}^{\prime \prime}(z)\right),
$$

hence, using Lemma 2.2(b):

$$
s_{i, e}^{\prime} u z=T_{i, e}^{\prime}(u)\left(s_{i, e}^{\prime} z\right), \quad s_{i, e}^{\prime \prime} u z=T_{i, e}^{\prime \prime}(u)\left(s_{i, e}^{\prime \prime} z\right) .
$$

We show:

$$
s_{i, e}^{\prime} s_{i,-e}^{\prime \prime}=1
$$

(c) for any $u \in \dot{\mathbf{U}}_{A}$ we have $T_{i, e}^{\prime}(u)=s_{i, e}^{\prime} u s_{i, e}^{\prime}{ }^{-1}, T_{i, e}^{\prime \prime}(u)=s_{i, e}^{\prime \prime} u s_{i, e}^{\prime \prime}{ }^{-1}$.

The proof of 2.3(b) and 2.3(c) is based on the following fact:

(d) Let $u^{\prime} \in \hat{\mathbf{U}}_{A}$ be such that $u^{\prime} z=0$ for any $M \in \mathfrak{C}_{A}$ and any $z \in M$. Then $u^{\prime}=0$.

We have $u^{\prime}=\sum_{a \in \dot{\mathbf{B}}} n_{a} a$ with $n_{a} \in A$. If $\lambda, \lambda^{\prime} \in X^{+}$, we have $u^{\prime}\left(\xi_{-\lambda} \otimes \eta_{\lambda}\right)=0$ in ${ }^{\omega} \Lambda_{\lambda, A} \otimes_{A} \Lambda_{\lambda^{\prime}, A}$. Hence $\sum_{a \in \dot{\mathbf{B}}_{\lambda, \lambda^{\prime}}} n_{a} a\left(\xi_{-\lambda} \otimes \eta_{\lambda}\right)=0$. Since the elements $a\left(\xi_{-\lambda} \otimes \eta_{\lambda}\right)$ with $a \in \dot{\mathbf{B}}_{\lambda, \lambda^{\prime}}$ form a basis of ${ }^{\omega} \Lambda_{\lambda, A} \otimes_{A} \Lambda_{\lambda^{\prime}, A}$, it follows that $n_{a}=0$ for any $a \in \dot{\mathbf{B}}_{\lambda, \lambda^{\prime}}$. Since $\dot{\mathbf{B}}=\bigcup_{\lambda, \lambda^{\prime}} \dot{\mathbf{B}}_{\lambda, \lambda^{\prime}}$, we see that $n_{a}=0$ for any $a \in \dot{\mathbf{B}}$, hence $u^{\prime}=0$. Thus, 2.3(d) holds.

We prove 2.3(b). Let $u^{\prime}=s_{i, e}^{\prime} s_{i,-e}^{\prime \prime}-1 \in \hat{\mathbf{U}}_{A}$. If $M \in \mathfrak{C}_{A}$ and $z \in M$, we have $u^{\prime} z=T_{i, e}^{\prime} T_{i,-e}^{\prime \prime}(z)-z=0$; see [L1, 41.2.4]. Using 2.3(d), we see that $u^{\prime}=0$ and $2.3(\mathrm{~b})$ is proved.

We prove 2.3(c). If $M \in \mathfrak{C}_{A}$ and $z \in M$, we have (using 2.3(b), (a)) $s_{i, e}^{\prime} u s_{i, e}^{\prime}{ }^{-1} s_{i, e}^{\prime} z$ $=s_{i, e}^{\prime} u z=T_{i, e}^{\prime}(u)(z)$. Thus, setting $u^{\prime}=s_{i, e}^{\prime} u s_{i, e}^{\prime}{ }^{-1}-T_{i, e}^{\prime}(u) \in \dot{\mathbf{U}}_{A}$, we have $u^{\prime} z=0$. Using 2.3(d), we see that $u^{\prime}=0$. This proves the first equality in 2.3(c). The second equality is proved similarly. 
2.4. Let $i \neq j$ in $I$. Let $e= \pm 1$. Let $n=n_{i j}=n_{j i}$ be as in 1.1. We show:

(a) $s_{i, e}^{\prime} s_{j, e}^{\prime} s_{i, e}^{\prime} \cdots=s_{j, e}^{\prime} s_{i, e}^{\prime} s_{j, e}^{\prime} \ldots$ in $\hat{\mathbf{U}}_{A}$

(b) $s_{i, e}^{\prime \prime} s_{j, e}^{\prime \prime} s_{i, e}^{\prime \prime} \cdots=s_{j, e}^{\prime \prime} s_{i, e}^{\prime \prime} s_{j, e}^{\prime \prime} \ldots$ in $\hat{\mathbf{U}}_{A}$

(all products in 2.4(a) and 2.4(b) have $n$ factors).

Let $s_{1} \in \hat{\mathbf{U}}_{A}$ be the left hand side minus the right hand side of 2.4(a). If $M \in \mathfrak{C}_{A}$ and $z \in M$, we have $s_{1} z=\left(T_{i, e}^{\prime} T_{j, e}^{\prime} T_{i, e}^{\prime} \ldots\right) z-\left(T_{j, e}^{\prime} T_{i, e}^{\prime} T_{j, e}^{\prime} \ldots\right) z$ and this is 0 by L1, 41.2.4(a)]. Thus $s_{1} z=0$. Using 2.3(d), we see that $s_{1}=0$. Thus 2.4(a) holds. The proof of 2.4(b) is similar.

2.5. Let $e= \pm 1$. Let $w \in W$. From $2.4(\mathrm{a})$, (b) we deduce by a standard argument that there are unique elements $w_{e}^{\prime} \in \hat{\mathbf{U}}_{A}, w_{e}^{\prime \prime} \in \hat{\mathbf{U}}_{A}$ such that $w_{e}^{\prime}=s_{i_{1}, e}^{\prime} s_{i_{2}, e}^{\prime} \ldots s_{i_{r}, e}^{\prime}$, $w_{e}^{\prime \prime}=s_{i_{1}, e}^{\prime \prime} s_{i_{2}, e}^{\prime \prime} \ldots s_{i_{r}, e}^{\prime \prime}$ for any sequence $i_{1}, i_{2}, \ldots, i_{r}$ in $W$ such that $w=s_{i_{1}} s_{i_{2}} \ldots s_{i_{r}}$ in $W$ with $r=l(w)$. Using 2.3(b), we see that $w_{e}^{\prime}\left(w^{-1}\right)_{-e}^{\prime \prime}=1$. From the definitions we have $\omega\left(w_{e}^{\prime}\right)=w_{e}^{\prime \prime}$.

2.6. Let $e= \pm 1$. Assume that $v=1$ in $A$. From Lemma 2.2(d) we see that for $i \in I, s_{i, e}^{\prime}, s_{i, e}^{\prime \prime}$ are independent of the choice of $e$; we denote them by $s_{i}^{\prime}, s_{i}^{\prime \prime}$. Using this and the definitions, we see that for $w \in W, w_{e}^{\prime}, w_{e}^{\prime \prime}$ are independent of the choice of $e$; we denote them by $w^{\prime}, w^{\prime \prime}$. Using Lemma 2.2(e) and the fact that $\hat{D}$ is an algebra homomorphism, we see that

(a) $\hat{D}\left(w^{\prime}\right)=w^{\prime} \otimes w^{\prime}, \hat{D}\left(w^{\prime \prime}\right)=w^{\prime \prime} \otimes w^{\prime \prime}\left(\right.$ in $\left.\hat{\mathbf{U}}_{A}^{(2)}\right)$ for any $w \in W$.

2.7. Let $\dot{\mathbf{B}}^{+}=\left\{b^{+} 1_{\lambda} ; b \in \mathbf{B}, \lambda \in X\right\}=\left\{1_{\lambda^{\prime}} b^{+} ; b \in \mathbf{B}, \lambda^{\prime} \in X\right\}$, a subset of $\dot{\mathbf{B}}$; see L1, 25.2.6]. Let $\dot{\mathbf{B}}^{-}=\left\{b^{-} 1_{\lambda} ; b \in \mathbf{B}, \lambda \in X\right\}=\left\{1_{\lambda^{\prime}} b^{-} ; b \in \mathbf{B}, \lambda^{\prime} \in X\right\}$, a subset of $\dot{\mathbf{B}}$. Let $\dot{\mathbf{U}}_{A}^{+}$(resp. $\dot{\mathbf{U}}_{A}^{-}$) be the $A$-submodule of $\dot{\mathbf{U}}_{A}$ spanned by $\dot{\mathbf{B}}^{+}$(resp. by $\left.\dot{\mathbf{B}}^{-}\right)$. Now $\dot{\mathbf{B}}^{+}$(resp. $\left.\dot{\mathbf{B}}^{-}\right)$is a basis of the $A$-module $\dot{\mathbf{U}}_{A}^{+}$(resp. $\dot{\mathbf{U}}_{A}^{-}$) and $\dot{\mathbf{U}}_{A}^{+}, \dot{\mathbf{U}}_{A}^{-}$ are subalgebras of $\dot{\mathbf{U}}_{A}$. From the definitions we have $\omega\left(b^{+} 1_{\lambda}\right)=b^{-} 1_{-\lambda}$ for $b \in \mathbf{B}$, $\lambda \in X$. Hence setting $a=b^{+} 1_{\lambda} \in \dot{\mathbf{B}}^{+}$, we have $e_{a}=1$ and $a^{!}=b^{-} 1_{-\lambda}$ (see 1.5). Thus, $\omega: \dot{\mathbf{U}}_{A} \rightarrow \dot{\mathbf{U}}_{A}$ restricts to a bijection $\dot{\mathbf{B}}^{+} \stackrel{\sim}{\longrightarrow} \dot{\mathbf{B}}^{-}$and to an $A$-algebra isomorphism $\dot{\mathbf{U}}_{A}^{+} \stackrel{\sim}{\longrightarrow} \dot{\mathbf{U}}_{A}^{-}$.

For $b, b^{\prime} \in \mathbf{B}$ we set $b^{+}=\sum_{\lambda \in X} b^{+} 1_{\lambda} \in \hat{\mathbf{U}}_{A}, b^{--}=\sum_{\lambda \in X} b^{\prime-} 1_{\lambda} \in \hat{\mathbf{U}}_{A}$. Then the elements $b^{+} 1_{\lambda} b^{\prime-} \in \dot{\mathbf{U}}_{A}, b^{\prime-} 1_{\lambda} b^{-} \in \dot{\mathbf{U}}_{A}$ (as in 1.4), which are not products in $\dot{\mathbf{U}}_{A}$, can be interpreted as products $b^{+} \cdot 1_{\lambda} \cdot b^{--}, b^{--} \cdot 1_{\lambda} \cdot b^{+}$in $\hat{\mathbf{U}}_{A}$.

2.8. Assume that $v=1$ in $A$. Let $w_{0}$ be as in 1.1. Let $n=l\left(w_{0}\right)$. We fix a sequence $i_{1}, i_{2}, \ldots, i_{n}$ in $I$ such that $s_{i_{1}} s_{i_{2}} \ldots s_{i_{n}}=w_{0}$. For any $c \in \mathbf{N}$ and $k \in[1, n]$ there is a unique element $x_{c, k} \in \mathbf{f}_{A}$ such that in $\dot{\mathbf{U}}_{A}$ we have

$$
T_{i_{1}, 1}^{\prime \prime} T_{i_{2}, 1}^{\prime \prime} \ldots T_{i_{k-1}, 1}^{\prime \prime}\left(\theta_{i_{k}}^{(c)+} 1_{s_{i_{k-1}} s_{i_{k-2}} \ldots s_{i_{1}} \lambda}\right)=x_{c, k}^{+} 1_{\lambda}
$$

for any $\lambda \in X^{+}$. (See [L1, 41.1.3].) For any $\mathbf{c}=\left(c_{1}, c_{2}, \ldots, c_{n}\right) \in \mathbf{N}^{n}$ we set

$$
x_{\mathbf{c}}=x_{c_{1}, 1} x_{c_{2}, 2} \ldots x_{c_{n}, n} \in \mathbf{f}_{A} .
$$

By [L1, 41.1.4], [L1, 41.1.7], the set $\left\{x_{\mathbf{c}} ; \mathbf{c} \in \mathbf{N}^{n}\right\}$ is an $A$-basis of $\mathbf{f}_{A}$. We show:

(a) For any $\mathbf{c} \in \mathbf{N}^{n}$ we have

$$
\hat{D}\left(x_{\mathbf{c}}^{+}\right)=\sum_{\mathbf{c}^{\prime}, \mathbf{c}^{\prime \prime} \in \mathbf{N} ; \mathbf{c}^{\prime}+\mathbf{c}^{\prime \prime}=\mathbf{c}} x_{\mathbf{c}^{\prime}}^{+} \otimes x_{\mathbf{c}^{\prime \prime}}^{+} \text {in } \hat{\mathbf{U}}^{(2)} .
$$


(The last sum is defined since $\mathbf{c}^{\prime}, \mathbf{c}^{\prime \prime}$ only take finitely many values.) For any $k \in[1, n], c \in \mathbf{N}$ we have

$$
\hat{D}\left(x_{c, k}^{+}\right)=\sum_{\substack{c^{\prime}, c^{\prime \prime} \in \mathbf{N} \\ c^{\prime}+c^{\prime \prime}=c}} x_{c^{\prime}, k}^{+} \otimes x_{c^{\prime \prime}, k}^{+} .
$$

(We use the formulas $T_{i, 1}^{\prime \prime}(u)=s_{i, 1}^{\prime \prime} u s_{i, 1}^{\prime \prime}{ }^{-1}$, see $2.3(\mathrm{c})$, and $\hat{D}\left(s_{i, 1}^{\prime \prime}\right)=s_{i, 1}^{\prime \prime} \otimes s_{i, 1}^{\prime \prime}$, see Lemma 2.2(e).) It follows that

$$
\begin{aligned}
& \hat{D}\left(x_{\mathbf{c}}^{+}\right)=\hat{D}\left(x_{c_{1}, 1}^{+}\right) \hat{D}\left(x_{c_{2}, 2}^{+}\right) \ldots \hat{D}\left(x_{c_{n}, n}^{+}\right) \\
& =\sum_{\mathbf{c}^{\prime}, \mathbf{c}^{\prime \prime} \in \mathbf{N} ; \mathbf{c}^{\prime}+\mathbf{c}^{\prime \prime}=\mathbf{c}}\left(x_{c_{1}^{\prime}, 1}^{+} x_{c_{2}^{\prime}, 2}^{+} \ldots x_{c_{n}^{\prime}, n}^{+}\right) \otimes\left(x_{c_{1}^{\prime \prime}, 1}^{+} x_{c_{2}^{\prime \prime}, 2}^{+} \ldots x_{c_{n}^{\prime \prime}, n}^{+}\right)
\end{aligned}
$$

which yields $2.8(\mathrm{a})$.

\section{The Hopf algebra $\mathbf{O}_{A}$}

3.1. In this section we define the Hopf algebra $\mathbf{O}_{A}$ as a submodule of the dual of $\dot{\mathbf{U}}_{A}$ defined in terms of $\dot{\mathbf{B}}$. We also study some basis properties of $\mathbf{O}_{A}$.

For any $A$-module $V$ we set $V^{\diamond}=\operatorname{Hom}_{A}(V, A)$. For any $a \in \dot{\mathbf{B}}$ we define a linear form $a^{*}: \dot{\mathbf{U}}_{A} \rightarrow A$ by $a^{\prime} \mapsto \delta_{a, a^{\prime}}$ for all $a^{\prime} \in \dot{\mathbf{B}}$. Let $\mathbf{O}_{A}$ be the $A$-submodule of $\dot{\mathbf{U}}_{A}^{\diamond}$ spanned by $\left\{a^{*} ; a \in \dot{\mathbf{B}}\right\}$. Thus $\left\{a^{*} ; a \in \dot{\mathbf{B}}\right\}$ is an $A$-basis of $\mathbf{O}_{A}$. We define an $A$-algebra structure on $\mathbf{O}_{A}$ by the rule $a^{*} b^{*}=\sum_{c \in \dot{\mathbf{B}}} \hat{m}_{c}^{a, b} c^{*}$ for any $a, b$ in $\mathbf{\mathbf { B }}$. (The sum is well defined by 1.16.) This algebra structure has a unit element, namely $1_{0}^{*}$. The $A$-linear map $\delta: \mathbf{O}_{A} \rightarrow \mathbf{O}_{A} \otimes_{A} \mathbf{O}_{A}$ given by $c^{*} \mapsto \sum_{(a, b) \in \dot{\mathbf{B}} \times \dot{\mathbf{B}}} m_{a, b}^{c} a^{*} \otimes b^{*}$ is well defined by 1.8; we call it comultiplication. Define an $A$-linear map (antipode) $S: \mathbf{O}_{A} \rightarrow \mathbf{O}_{A}$ by $S\left(a^{*}\right)=s_{a} \underline{a}^{*}$ (see 1.5) for $a \in \dot{\mathbf{B}}$. Using 1.5(i)-(vi), 1.5(ix)-(xii) we see that $\left(\mathbf{O}_{A}, \delta, S\right)$ is a Hopf algebra over $A$ with 1 whose counit $\mathbf{O}_{A} \rightarrow A$ is given by $a^{*} \mapsto 1$ if $a=1_{\lambda}$ for some $\lambda \in X$ and $a^{*} \mapsto 0$ if $a \in \dot{\mathbf{B}}$ is not of the form $1_{\lambda}, \lambda \in X$. From the definitions it is clear that $\mathbf{O}_{A}=A \otimes_{\mathcal{A}} \mathbf{O}_{\mathcal{A}}$ as a Hopf algebra. Define an $A$-linear involution $\omega: \mathbf{O}_{A} \rightarrow \mathbf{O}_{A}$ by $\omega\left(a^{*}\right)=e_{a} a^{! *}$ (see 1.5) for $a \in \dot{\mathbf{B}}$. This is an isomorphism of the algebra $\mathbf{O}_{A}$ onto the algebra $\mathbf{O}_{A}$ with the opposite multiplication (see 1.5(viii)) preserving 1; moreover it is compatible with the comultiplication of $\mathbf{O}_{A}$ (see 1.5(vii)).

3.2. We can reformulate 1.15 (a) as follows.

(a) Let $c \in \dot{\mathbf{B}}$. Assume that $\lambda, \lambda^{\prime}, \lambda_{1}, \lambda_{2}, \lambda_{1}^{\prime}, \lambda_{2}^{\prime}$ in $X^{+}$are such that $\lambda=\lambda_{1}+\lambda_{2}$, $\lambda^{\prime}=\lambda_{1}^{\prime}+\lambda_{2}^{\prime}, c \in \dot{\mathbf{B}}_{\lambda, \lambda^{\prime}}$. There exists a function $h: \dot{\mathbf{B}}_{\lambda_{1}, \lambda_{1}^{\prime}} \times \dot{\mathbf{B}}_{\lambda_{2}, \lambda_{2}^{\prime}} \rightarrow \mathcal{A}$ such that $\sum_{a \in \dot{\mathbf{B}}_{\lambda_{1}, \lambda_{1}^{\prime}}, b \in \dot{\mathbf{B}}_{\lambda_{2}, \lambda_{2}^{\prime}}} h(a, b) a^{*} b^{*}=c^{*}$ in $\mathbf{O}_{\mathcal{A}}$.

Here $a^{*} b^{*}$ is a product in $\mathbf{O}_{\mathcal{A}}$.

Proposition 3.3. The A-algebra $\mathbf{O}_{A}$ is finitely generated.

We choose $\lambda^{1}, \lambda^{2}, \ldots, \lambda^{r}$ in $X^{+}-\{0\}$ such that $X^{+}=\mathbf{N} \lambda^{1}+\mathbf{N} \lambda^{2}+\cdots+\mathbf{N} \lambda^{r}$. Let $\Gamma^{+}=\bigcup_{j \in[1, r]} \dot{\mathbf{B}}_{\lambda^{j}, 0}, \Gamma^{-}=\bigcup_{j \in[1, r]} \dot{\mathbf{B}}_{0, \lambda^{j}}, \Gamma=\Gamma^{+} \cup \Gamma^{-}$. We show:

(a) The (finite) set $\left\{b^{*} ; b \in \Gamma\right\}$ generates the A-algebra $\mathbf{O}_{A}$.

We can assume that $A=\mathcal{A}$. For any $c \in \dot{\mathbf{B}}$ let $N=N_{c}$ be the smallest integer $\geq 0$ such that there exist $\left(n_{1}, n_{2}, \ldots, n_{r}\right) \in \mathbf{N}^{r},\left(n_{1}^{\prime}, n_{2}^{\prime}, \ldots, n_{r}^{\prime}\right) \in \mathbf{N}^{r}$ with

(b) $c \in \dot{\mathbf{B}}_{\lambda, \lambda^{\prime}}$ where $\lambda=n_{1} \lambda^{1}+n_{2} \lambda^{2}+\cdots+n_{r} \lambda^{r}, \lambda^{\prime}=n_{1}^{\prime} \lambda^{1}+n_{2}^{\prime} \lambda^{2}+\cdots+n_{r}^{\prime} \lambda^{r}$ and $\sum_{j} n_{j}+\sum_{j} n_{j}^{\prime}=N$. 
Clearly $N_{c}$ is well defined. Let $\mathfrak{F}$ be the $\mathcal{A}$-subalgebra (with 1 ) of $\mathbf{O}_{\mathcal{A}}$ generated by $\Gamma$. It is enough to show that $c^{*} \in \mathfrak{F}$ for any $c \in \dot{\mathbf{B}}$. We shall prove this by induction on $N_{c}$. If $N_{c}=0$, then $c \in \dot{\mathbf{B}}_{0,0}$; hence $c=1_{0}$ and $c^{*}$ is the unit element of $\mathbf{O}_{\mathcal{A}}$. If $N_{c}=1$, then $c \in \Gamma$ and $c^{*} \in \mathfrak{F}$. Assume now that $N_{c}=N \geq 2$ and that the result is known for any $c^{\prime} \in \mathbf{B}$ with $N_{c^{\prime}}<N$. We can find $\lambda, \lambda^{\prime}$ in $X^{+}$and $\left(n_{j}\right) \in \mathbf{N}^{r},\left(n_{j}^{\prime}\right) \in \mathbf{N}^{r}$ so that (b) holds. We can find $\lambda_{1}, \lambda_{1}^{\prime}, \lambda_{2}, \lambda_{2}^{\prime}$ in $X^{+}$such that $\lambda_{1}+\lambda_{2}=\lambda, \lambda_{1}^{\prime}+\lambda_{2}^{\prime}=\lambda^{\prime}$ and either $\left(\lambda_{1}, \lambda_{1}^{\prime}\right)=\left(\lambda^{j}, 0\right)$ for some $j$ or $\left(\lambda_{1}, \lambda_{1}^{\prime}\right)=\left(0, \lambda^{j}\right)$ for some $j$. For any $a \in \dot{\mathbf{B}}_{\lambda_{1}, \lambda_{1}^{\prime}}$ we have $N_{a} \leq 1$; for any $b \in \dot{\mathbf{B}}_{\lambda_{2}, \lambda_{2}^{\prime}}$ we have $N_{b} \leq N-1$. We can write $c^{*}=\sum_{a \in \dot{\mathbf{B}}_{\lambda_{1}, \lambda_{1}^{\prime}}, b \in \dot{\mathbf{B}}_{\lambda_{2}, \lambda_{2}^{\prime}}} h(a, b) a^{*} b^{*}$ as in 3.2(a). By the induction hypothesis, for each $(a, b)$ in the sum we have $a^{*} \in \mathfrak{F}$, $b^{*} \in \mathfrak{F}$; hence $c^{*} \in \mathfrak{F}$. This completes the inductive proof of Proposition 3.3(a). The proposition is proved.

3.4. Let $\lambda \in X^{+}$. The sets $\dot{\mathbf{B}}_{\lambda, 0}, \dot{\mathbf{B}}_{0, \lambda}$ (see 1.9) can be described as follows:

$$
\dot{\mathbf{B}}_{\lambda, 0}=\left\{b^{+} 1_{-\lambda} ; b \in \mathbf{B}_{\lambda}^{\prime}\right\}, \quad \dot{\mathbf{B}}_{0, \lambda}=\left\{b^{-} 1_{\lambda} ; b \in \mathbf{B}_{\lambda}\right\} .
$$

We only prove the first of these equalities; the proof of the other equality is similar. For $b \in \mathbf{B}_{\lambda}^{\prime}$ we have $b^{+} 1_{-\lambda}\left(\xi_{-\lambda} \otimes \eta_{0}\right)=\left(b^{+} 1_{-\lambda} \xi_{-\lambda}\right) \otimes \eta_{0} \neq 0$. Since $b^{+} 1_{-\lambda} \in \dot{\mathbf{B}}$, it follows that $b^{+} 1_{-\lambda} \in \dot{\mathbf{B}}_{\lambda, 0}$. Thus $\left\{b^{+} 1_{-\lambda} ; b \in \mathbf{B}_{\lambda}^{\prime}\right\} \subset \dot{\mathbf{B}}_{\lambda, 0}$. Since this is an inclusion of finite sets with the same cardinal (equal to $\operatorname{dim} \Lambda_{\lambda}$ ), it is an equality, as required.

We see that:

(b) The number of generators of the A-algebra $\mathbf{O}_{A}$ given by the proof of Proposition 3.3 is at most $2 \sum_{s=1}^{r} \operatorname{dim} \Lambda_{\lambda^{s}}$.

3.5. For any $a \in \dot{\mathbf{B}}^{+}$(resp. $a \in \dot{\mathbf{B}}^{-}$) the restriction of the linear form $a^{*}: \dot{\mathbf{U}}_{A} \rightarrow A$ to $\dot{\mathbf{U}}_{A}^{+}$(resp. $\dot{\mathbf{U}}_{A}^{-}$) is denoted again by $a^{*}$. Let $\mathbf{O}_{A}^{+}$(resp. $\mathbf{O}_{A}^{-}$) be the $A$-submodule of $\dot{\mathbf{U}}_{A}^{+\diamond}\left(\right.$ resp. $\dot{\mathbf{U}}_{A}^{-\diamond}$ ) spanned by $\left\{a^{*} ; a \in \dot{\mathbf{B}}^{+}\right\}$(resp. $\left\{a^{*} ; a \in \dot{\mathbf{B}}^{-}\right\}$). Note that $\left\{a^{*} ; a \in \dot{\mathbf{B}}^{+}\right\}$(resp. $\left\{a^{*} ; a \in \dot{\mathbf{B}}^{-}\right\}$) is an $A$-basis of $\mathbf{O}_{A}^{+}$(resp. $\mathbf{O}_{A}^{-}$). We define an $A$-algebra structure on $\mathbf{O}_{A}^{ \pm}$by the rule $a^{*} b^{*}=\sum_{c \in \dot{\mathbf{B}}^{ \pm}} \hat{m}_{c}^{a, b} c^{*}$ for any $a, b$ in $\dot{\mathbf{B}}^{ \pm}$. (The sum is well defined by 1.16.) The (surjective) $A$-linear map $\pi^{+}: \mathbf{O}_{A} \rightarrow \mathbf{O}_{A}^{+}$ given by $a^{*} \mapsto a^{*}$ (if $a \in \dot{\mathbf{B}}^{+}$), $a^{*} \mapsto 0$ (if $a \in \dot{\mathbf{B}}-\dot{\mathbf{B}}^{+}$), respects the algebra structures. (If $c \in \dot{\mathbf{B}}^{+}, a \in \dot{\mathbf{B}}, b \in \dot{\mathbf{B}}, \hat{m}_{c}^{a, b} \neq 0$, then $a \in \dot{\mathbf{B}}^{+}, b \in \dot{\mathbf{B}}^{+}$.) Similarly, the (surjective) $A$-linear map $\pi^{-}: \mathbf{O}_{A} \rightarrow \mathbf{O}_{A}^{-}$given by $a^{*} \mapsto a^{*}\left(\right.$ if $\left.a \in \dot{\mathbf{B}}^{-}\right), a^{*} \mapsto 0$ (if $a \in \dot{\mathbf{B}}-\dot{\mathbf{B}}^{-}$) respects the algebra structures. It follows that the algebras $\mathbf{O}_{A}^{+}$, $\mathbf{O}_{A}^{-}$are associative with 1 . Define an $A$-linear map $\delta^{ \pm}: \mathbf{O}_{A}^{ \pm} \rightarrow \mathbf{O}_{A}^{ \pm} \otimes_{A} \mathbf{O}_{A}^{ \pm}$by

$$
\delta^{ \pm}\left(c^{*}\right)=\sum_{(a, b) \in \dot{\mathbf{B}}^{ \pm} \times \dot{\mathbf{B}}^{ \pm}} m_{a, b}^{c} a^{*} \otimes b^{*}
$$

for any $c \in \dot{\mathbf{B}}^{ \pm}$. The sum is well defined by 1.8. Note that $\pi^{ \pm}$is compatible with $\delta, \delta^{ \pm}$. Define an $A$-linear map $S^{ \pm}: \mathbf{O}_{A}^{ \pm} \rightarrow \mathbf{O}_{A}^{ \pm}$by $S^{ \pm}\left(a^{*}\right)=s_{a} \underline{a}^{*}$ for $a \in \dot{\mathbf{B}}^{ \pm}$. (We then have $\underline{a} \in \dot{\mathbf{B}}^{ \pm}$.) Note that $\left(\mathbf{O}_{A}^{ \pm}, \delta^{ \pm}, S^{ \pm}\right)$is a Hopf algebra over $A$ with 1 and with counit $a^{*} \mapsto 1$ if $a=1_{\lambda}$ for some $\lambda \in X$ and $a^{*} \mapsto 0$ if $a \in \dot{\mathbf{B}}^{ \pm}$is not of the form $1_{\lambda}, \lambda \in X$. From the definitions we have $\mathbf{O}_{A}^{ \pm}=A \otimes_{\mathcal{A}} \mathbf{O}_{\mathcal{A}}^{ \pm}$as Hopf algebras. Define an $A$-linear isomorphism $\omega: \mathbf{O}_{A}^{+} \stackrel{\sim}{\longrightarrow} \mathbf{O}_{A}^{-}$by $\omega\left(a^{*}\right)=a^{! *}$ (see 1.5) for $a \in \dot{\mathbf{B}}^{+}$. (Recall that $e_{a}=1$.) This is an isomorphism of the algebra $\mathbf{O}_{A}^{+}$onto the algebra $\mathbf{O}_{A}^{-}$with opposed multiplication. 
3.6. Let $\iota: \mathbf{O}_{A} \rightarrow \mathbf{O}_{A}^{-} \otimes_{A} \mathbf{O}_{A}^{+}$be the $A$-linear map given by the composition $\mathbf{O}_{A} \stackrel{\delta}{\longrightarrow}$ $\mathbf{O}_{A} \otimes_{A} \mathbf{O}_{A} \stackrel{\pi^{-} \otimes_{A} \pi^{+}}{\longrightarrow} \mathbf{O}_{A}^{-} \otimes_{A} \mathbf{O}_{A}^{+}$. (Note that $\iota$ is an $A$-algebra homomorphism.)

Lemma 3.7. The A-linear map $\iota$ is a split injection.

For $\lambda \in X^{+}$let $Z_{\lambda}$ be the set of pairs $(a, b) \in \dot{\mathbf{B}}^{-} \times \dot{\mathbf{B}}^{+}$such that $a \in \dot{\mathbf{B}}[\lambda], b \in$ $\dot{\mathbf{B}}[\lambda], a 1_{\lambda}=a, 1_{\lambda} b=b$. By [L2, 4.4] for any $(a, b) \in Z_{\lambda}$ there is a unique $c \in \dot{\mathbf{B}}[\lambda]$ such that $m_{a, b}^{c^{\prime}}=\delta_{c, c^{\prime}}$ for any $c^{\prime} \in \dot{\mathbf{B}}[\lambda]$; moreover $(a, b) \mapsto c$ is a bijection $\psi_{\lambda}$ : $Z_{\lambda} \stackrel{\sim}{\longrightarrow} \dot{\mathbf{B}}[\lambda]$. Let $Z=\bigsqcup_{\lambda \in X^{+}} Z_{\lambda}$. Then $\psi:=\bigsqcup_{\lambda} \psi_{\lambda}: Z \rightarrow \dot{\mathbf{B}}$ is a bijection.

Let $\underline{Z}$ be the $A$-submodule of $\mathbf{O}_{A}^{-} \otimes_{A} \mathbf{O}_{A}^{+}$spanned by $\left\{a^{*} \otimes b^{*} ;(a, b) \in Z\right\}$. Define an $A$-linear map $\rho: \mathbf{O}_{A}^{-} \otimes_{A} \mathbf{O}_{A}^{+} \rightarrow \underline{Z}$ by sending a basis element $a^{*} \otimes b^{*}$ to itself if $(a, b) \in Z$ and to 0 otherwise. It is enough to show that $\rho \iota$ is an isomorphism of $A$-modules.

Let $c \in \dot{\mathbf{B}}[\lambda], \lambda \in X^{+}$. By definition we have $\rho \iota\left(c^{*}\right)=\sum_{(a, b) \in Z} m_{a, b}^{c} a^{*} \otimes b^{*}$. If $(a, b) \in Z_{\lambda^{\prime}}, \lambda^{\prime} \in X^{+}$and $m_{a, b}^{c} \neq 0$, then as in the proof of Lemma 1.8 we have $\lambda^{\prime} \leq \lambda$. Thus

$$
\rho \iota\left(c^{*}\right)=a_{0}^{*} \otimes b_{0}^{*}+\sum_{\lambda^{\prime} \in X^{+} ; \lambda^{\prime}<\lambda} \sum_{(a, b) \in Z_{\lambda^{\prime}}} m_{a, b}^{c} a^{*} \otimes b^{*}
$$

where $\left(a_{0}, b_{0}\right)=\psi_{\lambda}^{-1}(c) \in Z_{\lambda}$. We identify $\underline{Z}$ with $\mathbf{O}_{A}$ as $A$-modules via the bijection $a^{*} \otimes b^{*} \mapsto c^{*}$ (with $c=\psi(a, b)$ ) between the bases of $\underline{Z}, \mathbf{O}_{A}$. Then we have

$$
\rho \iota\left(c^{*}\right)=c^{*}+\sum_{\lambda^{\prime} \in X^{+} ; \lambda^{\prime}<\lambda} \sum_{c^{\prime} \in \dot{\mathbf{B}}\left[\lambda^{\prime}\right]} x_{c^{\prime}, c} c^{\prime *}
$$

where $x_{c^{\prime}, c} \in A$. We see that $\rho \iota$ is represented by a square upper triangular matrix with entries in $A$ and with 1 on the diagonal. It follows that $\rho \iota$ is an isomorphism. The lemma is proved.

3.8. For $a, b \in \mathbf{B}$ we have $a b=\sum_{c \in \mathbf{B}} \mu_{a, b}^{c} c$ (in $\mathbf{f}_{A}$ ) where $\mu_{a, b}^{c} \in A$ are zero for all but finitely many $c$. In the case where $A=\mathcal{A}$ we define a homomorphism of $\mathcal{A}$-algebras $\underline{r}: \mathbf{f}_{\mathcal{A}} \rightarrow \mathbf{f}_{\mathcal{A}} \otimes_{\mathcal{A}} \mathbf{f}_{\mathcal{A}}$ as the restriction of the homomorphism $r: \mathbf{f} \rightarrow \mathbf{f} \otimes \mathbf{f}$ given in [L1, 1.2.6]. For general $A$ we define a homomorphism of $A$-algebras $\underline{r}$ : $\mathbf{f}_{A} \rightarrow \mathbf{f}_{A} \otimes_{A} \mathbf{f}_{A}$ by appplying $A \otimes_{\mathcal{A}}$ ? to the homomorphism $\underline{r}: \mathbf{f}_{\mathcal{A}} \rightarrow \mathbf{f}_{\mathcal{A}} \otimes_{\mathcal{A}} \mathbf{f}_{\mathcal{A}}$. For any $a, b, c$ in $\mathbf{B}$ we define $\hat{\mu}_{c}^{a, b} \in A$ by the following requirement: for any $c \in \mathbf{B}$ we have $\underline{r}(c)=\sum_{a, b \in \mathbf{B}} \hat{\mu}_{c}^{a, b} a \otimes b$ (in the last sum $\hat{\mu}_{c}^{a, b}$ is 0 for all but finitely many $(a, b))$.

In the rest of this section we assume that $v=1$ in $A$.

We define an $A$-algebra homomorphism $S$ from $\mathbf{f}_{A}$ to $\mathbf{f}_{A}$ with the opposite multiplication by $S\left(\theta_{i}^{(m)}\right)=(-1)^{m} \theta_{i}^{(m)}$ for all $i \in I, m \in \mathbf{N}$. For any $a \in \mathbf{B}$ we have $S(a)=q_{a} \tilde{a}$ where $a \mapsto \tilde{a}$ is an involution of $\mathbf{B}$ and $q_{a}= \pm 1$. Note that the quantities $\mu_{a, b}^{c}, \hat{\mu}_{c}^{a, b}, q_{a}$ (in $A$ ) are the images of the corresponding quantities in $\mathcal{A}$ under the given homomorphism $\mathcal{A} \rightarrow A$. Now $\left(\mathbf{f}_{A}, \underline{r}, S\right)$ is a Hopf algebra over $A$ with 1 whose counit $\mathbf{f}_{A} \rightarrow A$ is given by $a \mapsto \delta_{a, 1}$ for $a \in \dot{\mathbf{B}}$. For any $a \in \mathbf{B}$ let $a^{*}: \mathbf{f}_{A} \rightarrow A$ be the $A$-linear form given by $a^{*}(b)=\delta_{a, b}$ for $b \in \mathbf{B}$. Let $\mathbf{o}_{A}$ be the $A$-submodule of $\mathbf{f}_{A}^{\diamond}$ spanned by $\left\{a^{*} ; a \in \mathbf{B}\right\}$. Note that $\left\{a^{*} ; a \in \mathbf{B}^{+}\right\}$is an $A$-basis of $\mathbf{o}_{A}$. We define an $A$-algebra structure on $\mathbf{o}_{A}$ by the rule $a^{*} b^{*}=\sum_{c \in \mathbf{B}} \hat{\mu}_{c}^{a, b} c^{*}$ for any $a, b$ in $\mathbf{B}^{ \pm}$. (The sum is well defined by homogeneity reasons.) This algebra structure is associative with unit element $1^{*}$. Define an $A$-linear map (comultiplication) 
$\delta_{0}: \mathbf{o}_{A} \rightarrow \mathbf{o}_{A} \otimes_{A} \mathbf{o}_{A}$ by

$$
\delta_{0}\left(c^{*}\right)=\sum_{(a, b) \in \mathbf{B} \times \mathbf{B}} \mu_{a, b}^{c} a^{*} \otimes b^{*}
$$

for any $c \in \mathbf{B}$. (The sum is well defined by homogeneity reasons.) Define an $A$-linear map $S: \mathbf{o}_{A} \rightarrow \mathbf{o}_{A}$ (antipode) by $S\left(a^{*}\right)=q_{a} \tilde{a}^{*}$ for $a \in \mathbf{B}$. Note that $\left(\mathbf{o}_{A}, \delta_{0}, S\right)$ is a Hopf algebra over $A$ with 1 and with counit $a^{*} \mapsto \delta_{a, 1}$. From the definitions we have $\mathbf{o}_{A}=A \otimes_{\mathcal{A}} \mathbf{o}_{\mathcal{A}}$ as Hopf algebras. Define a (surjective) $A$-linear $\operatorname{map} \pi^{>0}: \mathbf{O}_{A} \rightarrow \mathbf{O}_{A}$ by $a^{*} \mapsto b^{*}$ if $a=b^{+} 1_{\lambda}$ for some $b \in \mathbf{B}, \lambda \in X$ and $a^{*} \mapsto 0$ if $a \in \dot{\mathbf{B}}$ is not of the form above. Define a (surjective) $A$-linear map $\pi^{<0}: \mathbf{O}_{A} \rightarrow \mathbf{O}_{A}$ by $a^{*} \mapsto b^{*}$ if $a=b^{-} 1_{\lambda}$ for some $b \in \mathbf{B}, \lambda \in X$ and $a^{*} \mapsto 0$ if $a \in \dot{\mathbf{B}}$ is not of the form above. Note that $\pi^{>0}$ and $\pi^{<0}$ respect the $A$-algebra structures and the unit elements. They are also compatible with $\delta, \delta_{0}$.

3.9. Let $A[X]$ be the group algebra of $X$ with coefficients in $A$. Define a (surjective) $A$-linear map $\pi^{0}: \mathbf{O}_{A} \rightarrow A[X]$ by $a^{*} \mapsto \lambda$ if $a=1_{\lambda}$ for some $\lambda \in X$ and $a^{*} \mapsto 0$ if $a \in \dot{\mathbf{B}}$ is not of this form. Note that $\pi^{0}$ respects the $A$-algebra structures and the unit elements.

Let $\iota^{\prime}: \mathbf{O}_{A} \rightarrow \mathbf{o}_{A} \otimes_{A} A[X] \otimes_{A} \mathbf{o}_{A}$ be the $A$-linear map given by the composition $\mathbf{O}_{A} \stackrel{(\delta \otimes 1) \delta}{\longrightarrow} \mathbf{O}_{A} \otimes_{A} \mathbf{O}_{A} \otimes_{A} \mathbf{O}_{A} \stackrel{\pi^{<0} \otimes_{A} \pi^{0} \otimes_{A} \pi^{>0}}{\longrightarrow} \mathbf{o}_{A} \otimes_{A} A[X] \otimes_{A} \mathbf{O}_{A}$. (Note that $\iota^{\prime}$ is an $A$-algebra homomorphism.) We have the following variant of Lemma 3.7:

Lemma 3.10. The A-linear map $\iota^{\prime}$ is a split injection. (Recall that $v=1$ in A.)

Let $Z_{\lambda}, \psi_{\lambda}, Z, \psi$ be as in 3.7. Let $\underline{Z}^{\prime}$ be the $A$-submodule of $\mathbf{o}_{A} \otimes_{A} A[X] \otimes_{A} \mathbf{o}_{A}$ spanned by $\left\{a_{1}^{*} \otimes \lambda \otimes b_{1}^{*} ;\left(a_{1}^{-} 1_{\lambda}, 1_{\lambda} b_{1}^{+}\right) \in Z\right\}$. Define an $A$-linear map

$$
\rho^{\prime}: \mathbf{o}_{A} \otimes_{A} A[X] \otimes_{A} \mathbf{o}_{A} \rightarrow \underline{Z}^{\prime}
$$

by sending a basis element $a_{1}^{*} \otimes \lambda \otimes b_{1}^{*}$ to itself if $\left(a_{1}^{-} 1_{\lambda}, 1_{\lambda} b_{1}^{+}\right) \in Z$ and to 0 otherwise. It is enough to show that $\rho^{\prime} \iota^{\prime}$ is an isomorphism of $A$-modules. Let $c \in \dot{\mathbf{B}}[\lambda], \lambda \in X^{+}$. By definition we have

$$
\rho^{\prime} \iota^{\prime}\left(c^{*}\right)=\sum_{\lambda^{\prime} \in X} \sum_{(a, b) \in Z_{\lambda^{\prime}}} m_{a, b}^{c} a_{1}^{*} \otimes \lambda^{\prime} \otimes b_{1}^{*}
$$

where $a_{1}, b_{1} \in \mathbf{B}$ are defined in terms of $a, b$ by $a=a_{1}^{-} 1_{\lambda}, b=1_{\lambda} b_{1}^{+}$. If $(a, b) \in Z_{\lambda^{\prime}}$, $\lambda^{\prime} \in X^{+}$and $m_{a, b}^{c} \neq 0$, then as in the proof of Lemma 1.8 we have $\lambda^{\prime} \leq \lambda$. Thus

$$
\rho^{\prime} \iota^{\prime}\left(c^{*}\right)=a_{0}^{*} \otimes \lambda \otimes b_{0}^{*}+\sum_{\lambda^{\prime} \in X^{+} ; \lambda^{\prime}<\lambda} \sum_{(a, b) \in Z_{\lambda^{\prime}}} m_{a, b}^{c} a_{1}^{*} \otimes \lambda^{\prime} \otimes b_{1}^{*}
$$

where $a_{0}, b_{0} \in \mathbf{B}$ are given by $\left(a_{0}^{-} 1_{\lambda}, 1_{\lambda} b_{0}^{+}\right)=\psi_{\lambda}^{-1}(c) \in Z_{\lambda}$ and $a_{1}, b_{1}$ are as above. We identify $\underline{Z}^{\prime}$ with $\mathbf{O}_{A}$ as $A$-modules via the bijection $a_{1}^{*} \otimes \lambda \otimes b_{1}^{*} \mapsto c^{*}$ (with $\left.c=\psi\left(a_{1}^{-} 1_{\lambda}, 1_{\lambda} b_{1}^{+}\right)\right)$between the bases of $\underline{Z}^{\prime}, \mathbf{O}_{A}$. Then we have

$$
\rho^{\prime} \iota^{\prime}\left(c^{*}\right)=c^{*}+\sum_{\lambda^{\prime} \in X^{+} ; \lambda^{\prime}<\lambda} \sum_{c^{\prime} \in \dot{\mathbf{B}}\left[\lambda^{\prime}\right]} x_{c^{\prime}, c}^{\prime} c^{*}
$$

where $x_{c^{\prime}, c}^{\prime} \in A$. We see that $\rho^{\prime} \iota^{\prime}$ is represented by a square upper triangular matrix with entries in $A$ and with 1 on the diagonal. It follows that $\rho^{\prime} \iota^{\prime}$ is an isomorphism. The lemma is proved. 
3.11. Let $\left\{x_{\mathbf{c}} ; \mathbf{c} \in \mathbf{N}^{n}\right\}$ be the $A$-basis of $\mathbf{f}_{A}$ defined in 2.8 . For any $\mathbf{c} \in \mathbf{N}^{n}$ we define an $A$-linear function $\xi_{\mathbf{c}}: \mathbf{f}_{A} \rightarrow A$ by $\xi_{\mathbf{c}}\left(x_{\mathbf{c}^{\prime}}\right)=\delta_{\mathbf{c}, \mathbf{c}^{\prime}}$ for any $\mathbf{c}^{\prime} \in \mathbf{N}^{n} \times X$.

Lemma 3.12. (a) $\left\{\xi_{\mathbf{c}} ; \mathbf{c} \in \mathbf{N}^{n}\right\}$ is an $A$-basis of $\mathbf{o}_{A}$.

(b) If $\mathbf{c}^{\prime}, \mathbf{c}^{\prime \prime} \in \mathbf{N}^{n}$, then $\xi_{\mathbf{c}^{\prime}} \xi_{\mathbf{c}^{\prime \prime}}=\xi_{\mathbf{c}^{\prime}+\mathbf{c}^{\prime \prime}}$, product in $\mathbf{o}_{A}$.

Note that each summand $\mathbf{f}_{\nu, A}$ of $\mathbf{f}_{A}$ is a free $A$-module of finite rank with basis given by its intersection with $\mathbf{B}$; this $A$-module also has as a basis its intersection with the $A$-basis $\left\{x_{\mathbf{c}} ; \mathbf{c} \in \mathbf{N}^{n}\right\}$ of $\mathbf{f}_{A}$. It follows that $\left\{\xi_{\mathbf{c}} ; \mathbf{c} \in \mathbf{N}^{n}\right\}$ and $\left\{b^{*} ; b \in \mathbf{B}\right\}$ span the same $A$-submodule of $\mathbf{f}_{A}^{\diamond}$, namely the set of $A$-linear functions $\mathbf{f}_{A} \rightarrow A$ which vanish on $\mathbf{f}_{\nu, A}$ for all but finitely many $\nu$. This proves Lemma 3.12(a).

We prove Lemma 3.12(b). For any $\mathbf{c} \in \mathbf{N}^{n}$ we can write uniquely $x_{\mathbf{c}}=$ $\sum_{b \in \mathbf{B}} h_{\mathbf{c}, b} b$ where $h_{\mathbf{c}, b} \in A$ are zero for all but finitely many $b$; for any $b \in \mathbf{B}$ we can write uniquely $b=\sum_{\mathbf{c} \in \mathbf{N}^{n}} \tilde{h}_{b, \mathbf{c}} x_{\mathbf{c}}$ where $\tilde{h}_{b, \mathbf{c}} \in A$ are zero for all but finitely many $\mathbf{c}$. We then have $\xi_{\mathbf{c}}=\sum_{b \in \mathbf{B}} \tilde{h}_{b, \mathbf{c}} b^{*}$ and $b^{*}=\sum_{\mathbf{c} \in \mathbf{N}^{n}} h_{\mathbf{c}, b} \xi_{\mathbf{c}}$. We have

$$
\xi_{\mathbf{c}^{\prime}} \xi_{\mathbf{c}^{\prime \prime}}=\left(\sum_{b^{\prime} \in \mathbf{B}} \tilde{h}_{b^{\prime}, \mathbf{c}^{\prime}} b^{\prime *}\right)\left(\sum_{b^{\prime \prime} \in \mathbf{B}} \tilde{h}_{b^{\prime \prime}, \mathbf{c}^{\prime \prime}} b^{\prime \prime *}\right)=\sum_{a, b^{\prime}, b^{\prime \prime} \in \mathbf{B}} \tilde{h}_{b^{\prime}, \mathbf{c}^{\prime}} \tilde{h}_{b^{\prime \prime}, \mathbf{c}^{\prime \prime}} \hat{\mu}_{a}^{b^{\prime}, b^{\prime \prime}} a^{*} ;
$$

hence Lemma 3.12(b) is equivalent to the set of equalities

$$
\sum_{b^{\prime}, b^{\prime \prime} \in \mathbf{B}} \tilde{h}_{b^{\prime}, \mathbf{c}^{\prime}} \tilde{h}_{b^{\prime \prime}, \mathbf{c}^{\prime \prime}} \hat{\mu}_{a}^{b^{\prime}, b^{\prime \prime}}=\tilde{h}_{a, \mathbf{c}^{\prime}+\mathbf{c}^{\prime \prime}}
$$

for any $\mathbf{c}^{\prime}, \mathbf{c}^{\prime \prime}, a$. The equality in $2.8($ a) can be rewritten as

$$
\underline{r}\left(x_{\mathbf{c}}\right)=\sum_{\mathbf{c}^{\prime}, \mathbf{c}^{\prime \prime} \in \mathbf{N} ; \mathbf{c}^{\prime}+\mathbf{c}^{\prime \prime}=\mathbf{c}} x_{\mathbf{c}^{\prime}} \otimes x_{\mathbf{c}^{\prime \prime}}
$$

hence as

$$
\sum_{a^{\prime}, a^{\prime \prime}, b \in \mathbf{B}} h_{\mathbf{c}, b} \hat{\mu}_{b}^{a^{\prime}, a^{\prime \prime}} a^{\prime} \otimes a^{\prime \prime}=\sum_{a^{\prime}, a^{\prime \prime} \in \mathbf{B}, \mathbf{c}^{\prime}, \mathbf{c}^{\prime \prime} \in \mathbf{N}^{n} ; \mathbf{c}^{\prime}+\mathbf{c}^{\prime \prime}=\mathbf{c}} h_{\mathbf{c}^{\prime}, a^{\prime}} h_{\mathbf{c}^{\prime \prime}, a^{\prime \prime}} a^{\prime} \otimes a^{\prime \prime} .
$$

Thus

$$
\sum_{b \in \mathbf{B}} h_{\mathbf{c}, b} \hat{\mu}_{b}^{a^{\prime}, a^{\prime \prime}}=\sum_{\mathbf{c}^{\prime}, \mathbf{c}^{\prime \prime} \in \mathbf{N}^{n} ; \mathbf{c}^{\prime}+\mathbf{c}^{\prime \prime}=\mathbf{c}} h_{\mathbf{c}^{\prime}, a^{\prime}} h_{\mathbf{c}^{\prime \prime}, a^{\prime \prime}}
$$

for any $a^{\prime}, a^{\prime \prime} \in \mathbf{B}, \mathbf{c} \in \mathbf{N}^{n}$. We multiply both sides by $\tilde{h}_{c, \mathbf{c}} \tilde{h}_{a^{\prime}, \mathbf{c}_{1}^{\prime}} \tilde{h}_{a^{\prime \prime}, \mathbf{c}_{1}^{\prime \prime}}$ and sum over all $\mathbf{c}, a^{\prime}, a^{\prime \prime}$. We obtain the equalities Lemma 3.12(c). This proves Lemma $3.12(\mathrm{~b})$. The lemma is proved.

Lemma 3.13. Assume that $v=1$ in $A$. Recall that $n=l\left(w_{0}\right)$. Let $A\left[t_{1}, t_{2}, \ldots, t_{n}\right]$ be the algebra of polynomials with coefficients in $A$ in the indeterminates $t_{1}, t_{2}, \ldots$, $t_{n}$. Define an A-linear map $\kappa: \mathbf{o}_{A} \rightarrow A\left[t_{1}, t_{2}, \ldots, t_{n}\right]$ by $\xi_{\mathbf{c}} \mapsto t_{1}^{c_{1}} t_{2}^{c_{2}} \ldots t_{n}^{c_{n}}$ for any $\mathbf{c}=\left(c_{1}, c_{2}, \ldots, c_{n}\right) \in \mathbf{N}^{n}$. Then $\kappa$ is an A-algebra isomorphism.

By Lemma 3.12(a), $\kappa$ is an $A$-linear isomorphism. It is compatible with the algebra structures by Lemma 3.12(b). The lemma is proved.

3.14. Define $\tilde{\iota}^{\prime}: \mathbf{O}_{A} \rightarrow A\left[t_{1}, t_{2}, \ldots, t_{n}\right] \otimes_{A} A[X] \otimes_{A} A\left[t_{1}, t_{2}, \ldots, t_{n}\right]$ as the composition $(\kappa \otimes 1 \otimes \kappa) \iota^{\prime}$ where $\iota^{\prime}: \mathbf{O}_{A} \rightarrow \mathbf{o}_{A} \otimes_{A} A[X] \otimes_{A} \mathbf{o}_{A}$ is as in 3.9.

Theorem 3.15. Recall the assumption that $v=1$ in $A$. The A-linear map $\tilde{\iota}^{\prime}$ is a split injection. It is also an imbedding of A-algebras with 1 . Thus, the A-algebra $\mathbf{O}_{A}$ is isomorphic to a finitely generated $A$-subalgebra (with 1 ) of $A\left[t_{1}, t_{2}, \ldots, t_{n}\right] \otimes_{A}$ $A[X] \otimes_{A} A\left[t_{1}, t_{2}, \ldots, t_{n}\right]$. In particular, if $A$ is an integral domain, then $\mathbf{O}_{A}$ is an integral domain. 
The fact that $\tilde{\iota}^{\prime}$ is a split injection follows from 3.10 and the fact that $\kappa \otimes 1 \otimes \kappa$ is an isomorphism of $A$-modules. The fact that $\tilde{\iota}^{\prime}$ is an algebra homomorphism follows from the fact that $\iota^{\prime}$ is an algebra homomorphism and that $\kappa$ is an algebra isomorphism. The fact that the algebra $\mathbf{O}_{A}$ is finitely generated follows from Proposition 3.3. If $A$ is an integral domain, then $A\left[t_{1}, t_{2}, \ldots, t_{n}\right] \otimes_{A} A[X] \otimes_{A} A\left[t_{1}, t_{2}, \ldots, t_{n}\right]$ is an integral domain and hence so is $\mathbf{O}_{A}$. The theorem is proved.

\section{The group $G_{A}$}

4.1. In this section we assume that $v=1$ in $A$.

In analogy with $\mathrm{Ko}$, we define $G_{A}$ as the set of $A$-algebra homomorphisms $\mathbf{O}_{A} \rightarrow A$ which take 1 to 1 . Since $\mathbf{O}_{A}$ is a Hopf algebra over $A$ with 1 and with counit, $G_{A}$ has a natural group structure. We now describe the group strcture of $G_{A}$ in more detail. Note that $G_{A}$ is the set of all $A$-linear functions $\phi: \mathbf{O}_{A} \rightarrow A$ such that $\phi\left(a^{*} b^{*}\right)=\phi\left(a^{*}\right) \phi\left(b^{*}\right)$ for all $a, b \in \dot{\mathbf{B}}$ and such that $\phi\left(1_{0}^{*}\right)=1$. By the correspondence $\phi \mapsto \sum_{a \in \dot{\mathbf{B}}} \phi\left(a^{*}\right) a$ we identify $G_{A}$ with the subset of $\hat{\mathbf{U}}_{A}$ defined as

$$
\left\{\sum_{a \in \dot{\mathbf{B}}} n_{a} a \in \hat{\mathbf{U}}_{A} ; n_{1_{0}}=1, \sum_{c \in \dot{\mathbf{B}}} \hat{m}_{c}^{a, b} n_{c}=n_{a} n_{b} \text { for all } a, b \in \dot{\mathbf{B}}\right\}
$$

or equivalently as $\left\{\xi \in \hat{\mathbf{U}}_{A} ; \hat{D}(\xi)=\xi \otimes \xi, \epsilon(\xi)=1\right\}$. Note that $G_{A}$ is closed under the multiplication in the algebra $\hat{\mathbf{U}}_{A}$. Thus the multiplication in $\hat{\mathbf{U}}_{A}$ induces an associative monoid structure on $G_{A}$. Since the unit element 1 of $\hat{\mathbf{U}}_{A}$ satisfies $\hat{D}(1)=1 \otimes 1, \epsilon(1)=1$, it belongs to $G_{A}$ and plays the role of a unit element for the monoid structure of $G_{A}$. If $\xi \in G_{A}$, we have $\hat{D}(S(\xi))=S^{(2)}(\hat{D}(\xi))=S^{(2)}(\xi \otimes \xi)=$ $S(\xi) \otimes S(\xi)$ and $\epsilon(S(\xi))=\epsilon(\xi)=1$. Thus $S(\xi) \in G_{A}$. From 1.5(xi) we have $\mathbf{m}\left(S^{(0,1)}(\hat{D}(\xi))\right)=\xi$. In our case this can be written as $\mathbf{m}\left(S^{(0,1)}(\xi \otimes \xi)\right)=\xi$, that is, $\mathbf{m}(\xi \otimes S(\xi))=\xi$ and $\xi S(\xi)=\xi$. We see that any element of $G_{A}$ has a right inverse in the monoid $G_{A}$. Hence any element of $G_{A}$ has a left inverse in the monoid $G_{A}$. Thus $G_{A}$ is a group (a subgroup of the group of invertible elements of the algebra $\hat{\mathbf{U}}_{A}$ ). From the definitions we see that the algebra involution $\omega: \hat{\mathbf{U}}_{A} \rightarrow \hat{\mathbf{U}}_{A}$ restricts to an involution $\omega: G_{A} \rightarrow G_{A}$ which is a group isomorphism.

4.2. Let $\hat{\mathbf{U}}_{A}^{>0}$ (resp. $\hat{\mathbf{U}}_{A}^{<0}$ ) be the $A$-submodule of $\hat{\mathbf{U}}_{A}$ consisting of all elements of the form $\sum_{b \in \mathbf{B}, \lambda \in X} n_{b}\left(1_{\lambda} b^{+}\right)$(resp. $\sum_{b \in \mathbf{B}, \lambda \in X} n_{b}\left(b^{-} 1_{\lambda}\right)$ ) where $n_{b} \in A$. Let $\mathfrak{G}_{A}$ be the set of $A$-algebra homomorphisms $\mathbf{o}_{A} \rightarrow A$ which take 1 to 1 . Since $\mathbf{o}_{A}$ is a Hopf algebra over $A$ with 1 and with counit, $\mathfrak{G}_{A}$ has a natural group structure. Note that $\mathfrak{G}_{A}$ is the set of $A$-linear maps $\phi: \mathbf{o}_{A} \rightarrow A$ such that $\phi\left(a^{*} b^{*}\right)=\phi\left(a^{*}\right) \phi\left(b^{*}\right)$ for all $a, b \in \mathbf{B}$ and such that $\phi(1)=1$. (Here $a^{*} b^{*}$ is the product in $\mathbf{o}_{A}$.) By the correspondence $\phi \mapsto \sum_{a \in \mathbf{B}, \lambda \in X} \phi\left(a^{*}\right) a^{-} 1_{\lambda}$ we identify $\mathfrak{G}_{A}$ with the set of all $\xi \in \hat{\mathbf{U}}_{A}^{<0}$ such that $\hat{D}(\xi)=\xi \otimes \xi\left(\right.$ in $\hat{\mathbf{U}}_{A}^{(2)}$ ) and $\epsilon(\xi)=1$ or equivalently with $G_{A}^{<0}:=G_{A} \cap \hat{\mathbf{U}}_{A}^{<0}$. Similarly, by the correspondence $\phi \mapsto \sum_{a \in \mathbf{B}, \lambda \in X} \phi\left(a^{*}\right) 1_{\lambda} a^{+}$ we identify $\mathfrak{G}_{A}$ with the set of all $\xi \in \hat{\mathbf{U}}_{A}^{>0}$ such that $\hat{D}(\xi)=\xi \otimes \xi\left(\right.$ in $\left._{\mathbf{U}_{A}^{(2)}}\right)$ and $\epsilon(\xi)=1$ or equivalently with $G_{A}^{>0}:=G_{A} \cap \hat{\mathbf{U}}_{A}^{>0}$. The group structure on $\mathfrak{G}_{A}$ coincides with that induced from the group structure of $G_{A}$ via either one of the isomorphisms $\mathfrak{G}_{A} \stackrel{\sim}{\sim} G_{A}^{<0}, \mathfrak{G}_{A} \stackrel{\sim}{\longrightarrow} G_{A}^{>0}$.

Let $\hat{\mathbf{U}}_{A}^{0}$ be the set of elements of $\hat{\mathbf{U}}_{A}$ of the form $\sum_{\lambda \in X} n_{\lambda} 1_{\lambda}$ with $n_{\lambda} \in A$. Let $T_{A}=\hat{\mathbf{U}}_{A}^{0} \cap G_{A}$. Thus $T_{A}$ is the set of elements of $\hat{\mathbf{U}}_{A}$ of the form $\sum_{\lambda \in X} n_{\lambda} 1_{\lambda}$ with $n_{\lambda} \in A^{\circ}$ such that $\lambda \mapsto n_{\lambda}$ is a group homomorphism $X \rightarrow A^{\circ}$. Clearly, $T_{A}$ 
is an abelian subgroup of $G_{A}$. Note that $T_{A}$ can be identified with $\operatorname{Hom}\left(X, A^{\circ}\right)=$ $A^{\circ} \otimes \mathbf{z} Y$. The isomorphism between $A^{\circ} \otimes Y$ and $T_{A}$ associates to $d \otimes y$ (where $d \in A^{\circ}$, $y \in Y$ ) the element $\sum_{\lambda \in X} d^{\langle y, \lambda\rangle} 1_{\lambda}$ of $T_{A}$. (Since $d \in A^{\circ}$, it can be raised to any integer power.) Note that $T_{A}$ can also be identified with the set of homomorphisms of $A$-algebras $A[X] \rightarrow A$ preserving 1 , by $\left[g=\sum_{\lambda \in X} n_{\lambda} 1_{\lambda} \in T_{A}\right] \mapsto\left[\lambda \mapsto n_{\lambda}\right]$. We show:

(a) Multiplication in $G_{A}$ defines an injective $\operatorname{map} G_{A}^{<0} \times T_{A} \times G_{A}^{>0} \rightarrow G_{A}$.

Let $\hat{\mathbf{U}}_{A}^{-}$be the set of elements of $\hat{\mathbf{U}}_{A}$ of the form $\sum_{a \in \dot{\mathbf{B}}^{-}} n_{a} a$. Let $\xi_{1}, \xi_{1}^{\prime} \in G_{A}^{<0}$, $\xi_{2}, \xi_{2}^{\prime} \in T_{A}, \xi_{3}, \xi_{3}^{\prime} \in G_{A}^{>0}$ be such that $\xi_{1} \xi_{2} \xi_{3}=\xi_{1}^{\prime} \xi_{2}^{\prime} \xi_{3}^{\prime}$ in $G_{A}$. Then $\xi_{3}^{\prime} \xi_{3}^{-1}=$ $\xi_{2}^{\prime-1} \xi_{1}^{\prime-1} \xi_{1} \xi_{2}$. The right hand side is contained in $\hat{\mathbf{U}}_{A}^{-}$since $G_{A}^{<0}, T_{A}$ are contained in $\hat{\mathbf{U}}_{A}^{-}$and $\hat{\mathbf{U}}_{A}^{-}$is closed under multiplication in $\hat{\mathbf{U}}_{A}$. Thus we have $\xi_{3}^{\prime} \xi_{3}^{-1} \in$ $\hat{\mathbf{U}}_{A}^{-}$. Since $G_{A}^{>0} \in \hat{\mathbf{U}}_{A}^{>0}$ and $\hat{\mathbf{U}}_{A}^{>0}$ is closed under multiplication in $\hat{\mathbf{U}}_{A}$, we also have $\xi_{3}^{\prime} \xi_{3}^{-1} \in \hat{\mathbf{U}}_{A}^{>0}$. Thus $\xi_{3}^{\prime} \xi_{3}^{-1} \in \hat{\mathbf{U}}_{A}^{-} \cap \hat{\mathbf{U}}_{A}^{>0}$. From the definitions we have $\hat{\mathbf{U}}_{A}^{-} \cap \hat{\mathbf{U}}_{A}^{>0}=\{1\}$. Thus $\xi_{3}^{\prime} \xi_{3}^{-1}=1$ so that $\xi_{3}^{\prime}=\xi_{3}$. It follows that $\xi_{1} \xi_{2}=\xi_{1}^{\prime} \xi_{2}^{\prime}$. Then $x_{1}^{\prime-1} x_{1}=x_{2}^{\prime} x_{2}^{-1}$ belongs to both $\hat{\mathbf{U}}_{A}^{<0}$ and to $\hat{\mathbf{U}}_{A}^{0}$ which have intersection $\{1\}$. Thus $x_{1}^{\prime-1} x_{1}=x_{2}^{\prime} x_{2}^{-1}=1$ and 4.2 (a) follows.

From the definitions we see that the involution $\omega: G_{A} \rightarrow G_{A}$ interchanges $G_{A}^{>0}$ with $G_{A}^{<0}$ and its restriction to $T_{A}$ is given by $g \mapsto g^{-1}$.

4.3. For any $i \in I, h \in A$ we have $x_{i}(h) \in G_{A}^{>0}, y_{i}(h) \in G_{A}^{<0}$. (We use 1.18(a).)

For any $w \in W$, the elements $w^{\prime}, w^{\prime \prime}$ of $\hat{\mathbf{U}}_{A}$ (see $2.5,2.6$ ) belong to $G_{A}$. (We use 2.6(a).) We have $w^{\prime}\left(w^{-1}\right)^{\prime \prime}=1$; see 2.5.

Now $W$ acts on $T_{A}$ by $w: \sum_{\lambda \in X} n_{\lambda} 1_{\lambda} \mapsto \sum_{\lambda \in X} n_{w^{-1}(\lambda)} 1_{\lambda}$. This corresponds to the $W$-action on $A^{\circ} \otimes Y$ given by $w: d \otimes y \mapsto d \otimes w(y)$. This $W$ action on $T_{A}$ is denoted by $w: g \mapsto w(g)$. We show:

(a) If $w \in W, g \in T_{A}$, then $w(g)=w^{\prime} g w^{\prime-1}=w^{\prime \prime} g w^{\prime \prime-1}$.

Using the definition of $w^{\prime}$, we see that to prove the first equality in 4.3(a) we may assume that $w=s_{i}$ for some $i \in I$. In this case it is enough to show that $1_{s_{i}(\lambda)}=s_{i}^{\prime} 1_{\lambda}\left(s_{i}^{\prime}\right)^{-1}$ or that $1_{s_{i}(\lambda)}=T_{i, 1}^{\prime}\left(1_{\lambda}\right)$ for any $\lambda \in X$. This follows from [L1, 41.1.2]. The proof of the second equality in 4.3(a) is similar.

4.4. Let $i \in I, u \in A^{\circ}$. Let $t_{i}(u)=\sum_{\lambda \in X} u^{\langle i, \lambda\rangle} 1_{\lambda} \in T_{A}$. We show:

(a) $s_{i}^{\prime \prime}=s_{i}^{\prime} t_{i}(-1)$ in $G_{A}$.

We have

$$
s_{i}^{\prime} t_{i}(-1)=\sum_{y \in \mathbf{Z}, x \in \mathbf{Z}, \lambda, \lambda^{\prime} \in X ; \lambda^{\prime}=\lambda-y i^{\prime},\langle i, \lambda\rangle=x+y}(-1)^{y}(-1)^{\left\langle i, \lambda^{\prime}\right\rangle} \theta_{i}^{(x)-} 1_{\lambda} \theta_{i}^{(y)+} .
$$

It is enough to show that $(-1)^{y+\left\langle i, \lambda-y i^{\prime}\right\rangle}=(-1)^{x}$ in the sum above. This follows from $\left\langle i, i^{\prime}\right\rangle=2$ and $y+\langle i, \lambda\rangle=x \bmod 2$.

4.5. Let $i \in I$. Let $e, f, g \in A$ be such that $d:=e g+f^{2} \in A^{\circ}, f \in A^{\circ}$. Define $e^{\prime}, f^{\prime}, g^{\prime} \in A$ by $e^{\prime}=e d^{-1}, f^{\prime}=f d^{-1}, g^{\prime}=g d^{-1}$. Note that $d^{\prime}:=e^{\prime} g^{\prime}+f^{\prime 2}=d^{-1} \in$ $A^{\circ}, f^{\prime} \in A^{\circ}$ and $e=e^{\prime} d^{\prime-1}, f=f^{\prime} d^{\prime-1}, g=g^{\prime} d^{\prime-1}$. We show:

$$
y_{i}(e) t_{i}\left(f^{-1}\right) x_{i}(g)=x_{i}\left(g^{\prime}\right) t_{i}\left(f^{\prime}\right) y_{i}\left(e^{\prime}\right) .
$$


We compute the left hand side in $\hat{\mathbf{U}}_{A}$ :

$$
\begin{aligned}
& \sum_{\substack{c, c^{\prime} \in \mathbf{N}, \lambda \in X}} e^{c} f^{-\langle i, \lambda\rangle} g^{c^{\prime}} \theta_{i}^{(c)-} 1_{\lambda} \theta_{i}^{\left(c^{\prime}\right)+}=\sum_{\substack{c, c^{\prime} \in \mathbf{N}, \lambda \in X \\
\langle i, \lambda\rangle \geq c+c^{\prime}}} e^{c} f^{-\langle i, \lambda\rangle} g^{c^{\prime}} \theta_{i}^{(c)-} 1_{\lambda} \theta_{i}^{\left(c^{\prime}\right)+} \\
& +\sum_{\substack{c, c^{\prime}, t \in \mathbf{N}, \lambda \in X \\
\langle i, \lambda\rangle<c+c^{\prime}}} e^{c} f^{-\langle i, \lambda\rangle} g^{c^{\prime}}\left(\begin{array}{c}
c+c^{\prime}-\langle i, \lambda\rangle \\
t
\end{array}\right) \theta_{i}^{\left(c^{\prime}-t\right)+} 1_{\lambda-\left(c+c^{\prime}-t\right) i^{\prime}} \theta_{i}^{\left(c^{\prime}-t\right)-} \\
& =\sum_{\substack{c, c^{\prime} \in \mathbf{N}, \lambda \in X ;\langle i, \lambda\rangle \geq c+c^{\prime} \\
+}} \sum^{c} f^{-\langle i, \lambda\rangle} g^{c^{\prime}} \theta_{i}^{(c)-} 1_{\lambda} \theta_{i}^{\left(c^{\prime}\right)+} \\
& =\sum_{\substack{\left\langle, r^{\prime} \in \mathbf{N}, \lambda^{\prime}\right\rangle>\lambda^{\prime} \in X \\
c, c^{\prime} \in \mathbf{N}, \lambda \in X \in r^{\prime}}} \sum_{\substack{t \in \mathbf{N} \\
+}} e^{r^{\prime}+t} f^{-\left\langle i, \lambda^{\prime}+\left(r+r^{\prime}+t\right) i^{\prime}\right\rangle} g^{r+t}\left(\begin{array}{c}
-r-r^{\prime}-\left\langle i, \lambda^{\prime}\right\rangle \\
t
\end{array}\right) \theta_{i}^{(r)+} 1_{\lambda^{\prime}} \theta_{i}^{\left(r^{\prime}\right)-} \\
& \sum_{\substack{r, r^{\prime} \in \mathbf{N}, \lambda^{\prime} \in X ; \\
\left\langle i,-\lambda^{\prime}\right\rangle>r+r^{\prime}}} e^{r^{\prime}} e^{-\left\langle i, \lambda^{\prime}+\left(r+r^{\prime}\right) i^{\prime}\right\rangle} g^{r}\left(1+e f^{-2} g\right)^{-r-r^{\prime}-\left\langle i, \lambda^{\prime}\right\rangle} \theta_{i}^{(r)+} 1_{\lambda^{\prime}} \theta_{i}^{\left(r^{\prime}\right)-} .
\end{aligned}
$$

We compute the right hand side of $4.5(\mathrm{a})$ in $\hat{\mathbf{U}}_{A}$ :

$$
\begin{aligned}
& \sum_{\substack{c, c^{\prime} \in \mathbf{N}, \lambda \in X}} g^{\prime c} f^{\prime\langle i, \lambda\rangle} e^{\prime c^{\prime}} \theta_{i}^{(c)+} 1_{\lambda} \theta_{i}^{\left(c^{\prime}\right)-}=\sum_{\substack{c, c^{\prime} \in \mathbf{N}, \lambda \in X ; \\
-\langle i, \lambda\rangle>c+c^{\prime}}} g^{\prime c} f^{\prime\langle i, \lambda\rangle} e^{\prime c^{\prime}} \theta_{i}^{(c)+} 1_{\lambda} \theta_{i}^{\left(c^{\prime}\right)-} \\
& +\sum_{\substack{c, c^{\prime}, t \in \mathbf{N}, \lambda \in X ; \\
-\langle i, \lambda\rangle \leq c+c^{\prime}}} g^{\prime c} f^{\prime\langle i, \lambda\rangle} e^{\prime c^{\prime}}\left(\begin{array}{c}
c+c^{\prime}+\langle i, \lambda\rangle \\
t
\end{array}\right) \theta_{i}^{\left(c^{\prime}-t\right)-} 1_{\lambda+\left(c+c^{\prime}-t\right) i^{\prime}} \theta_{i}^{(c-t)+} \\
& =\sum_{\left.c, c^{\prime} \in \mathbf{N}, \lambda \in X ;-\langle i, \lambda\rangle\right\rangle c+c^{\prime}} g^{\prime c} f^{\prime\langle i, \lambda\rangle} e^{\prime c^{\prime}} \theta_{i}^{(c)+} 1_{\lambda} \theta_{i}^{\left(c^{\prime}\right)-} \\
& +\sum_{\substack{s, s^{\prime} \in \mathbf{N}, \lambda^{\prime} \in X \\
\left\langle i, \lambda^{\prime}\right\rangle \geq s+s^{\prime}}} \sum_{t \in \mathbf{N}} g^{\prime s^{\prime}+t} f^{\prime\left\langle i, \lambda^{\prime}-(s+s+t) i^{\prime}\right\rangle} e^{s+t}\left(\begin{array}{c}
-s-s^{\prime}+\left\langle i, \lambda^{\prime}\right\rangle \\
t
\end{array}\right) \theta_{i}^{(s)-} 1_{\lambda^{\prime}} \theta_{i}^{\left(s^{\prime}\right)+} \\
& =\sum_{c, c^{\prime} \in \mathbf{N}, \lambda \in X ;-\langle i, \lambda\rangle>c+c^{\prime}} g^{\prime c} f^{\prime\langle i, \lambda\rangle} e^{\prime c^{\prime}} \theta_{i}^{(c)+} 1_{\lambda} \theta_{i}^{\left(c^{\prime}\right)-} \\
& +\sum_{\substack{s, s^{\prime} \in \mathbf{N}, \lambda^{\prime} \in X \\
\left\langle i, \lambda^{\prime}\right\rangle \geq s+s^{\prime}}} g^{\prime s^{\prime}} f^{\prime\left\langle i, \lambda^{\prime}-(s+s) i^{\prime}\right\rangle} e^{s}\left(1+g^{\prime} f^{\prime-2} e^{\prime}\right)^{-s-s^{\prime}+\left\langle i, \lambda^{\prime}\right\rangle} \theta_{i}^{(s)-} 1_{\lambda^{\prime}} \theta_{i}^{\left(s^{\prime}\right)+} .
\end{aligned}
$$

To complete the proof, it is enough to show that

$$
\begin{aligned}
& e^{c} f^{-\langle i, \lambda\rangle} g^{c^{\prime}}=g^{\prime c^{\prime}} f^{\prime\left\langle i, \lambda-\left(c+c^{\prime}\right) i^{\prime}\right\rangle} e^{\prime c}\left(1+g^{\prime} f^{\prime-2} e^{\prime}\right)^{-c-c^{\prime}+\langle i, \lambda\rangle}, \\
& g^{\prime c} f^{\prime\langle i, \lambda\rangle} e^{\prime c^{\prime}}=e^{c^{\prime}} f^{-\left\langle i, \lambda+\left(c+c^{\prime}\right) i^{\prime}\right\rangle} g^{c}\left(1+e f^{-2} g\right)^{-c-c^{\prime}-\langle i, \lambda\rangle}
\end{aligned}
$$

for any $c, c^{\prime} \in \mathbf{N}, \lambda \in X$. This is immediate.

4.6. Let $i \in I$ and let $u \in A^{\circ}$. We have

$$
x_{i}(u-1) y_{i}(1) x_{i}\left(u^{-1}-1\right) y_{i}(-u)=t_{i}(u) \in T_{A} .
$$


Taking $e=1, f=1, g=u^{-1}-1$ in $4.5(\mathrm{a})$, we obtain

$$
y_{i}(1) x_{i}\left(u^{-1}-1\right)=x_{i}(-u+1) t_{u} y_{i}(u)
$$

and 4.6(a) follows.

4.7. Let $w \in W, i \in I$ be such that $l\left(w s_{i}\right)=l(w)+1$. Let $h \in A$. We show:

(a) $w^{\prime \prime} x_{i}(h) w^{\prime \prime-1} \in G_{A}^{>0}$.

We can find a sequence $i_{1}, i_{2}, \ldots, i_{n}$ in $I$ as in 2.8 such that $w=s_{i_{1}} s_{i_{2}} \ldots s_{i_{k-1}}$, $i=i_{k}, k-1=l(w)$. The equality

$$
T_{i_{1}, 1}^{\prime \prime} T_{i_{2}, 1}^{\prime \prime} \ldots T_{i_{k-1}, 1}^{\prime \prime}\left(\theta_{i_{k}}^{(c)+} 1_{s_{i_{k-1}} s_{i_{k-2}} \ldots s_{i_{1}} \lambda}\right)=x_{c, k}^{+} 1_{\lambda}
$$

in 2.8 (with $c \in \mathbf{N}, \lambda \in X$, and with $x_{c, k} \in \mathbf{f}_{A}$ independent of $\lambda$ ) can be rewritten as

$$
w^{\prime \prime}\left(\theta_{i_{k}}^{(c)+} 1_{s_{i_{k-1}} s_{i_{k-2}} \ldots s_{i_{1}} \lambda}\right) w^{\prime \prime-1}=x_{c, k}^{+} 1_{\lambda} .
$$

(See 2.3(c).) We multiply this by $h^{c}$ and sum over all $\lambda \in X$ and $c \in \mathbf{N}$; we obtain

$$
w^{\prime \prime} x_{i}(h) w^{\prime \prime-1}=\sum_{c \in \mathbf{N}} h^{c} x_{c, k}^{+} .
$$

The sum in the right hand side is well defined since for some $\nu \in \mathbf{N}[I]-\{0\}$ we have $x_{c, k}^{+} \in \mathbf{f}_{c \nu, A}$. Thus we have $w^{\prime \prime} x_{i}(h) w^{\prime \prime-1} \in \hat{\mathbf{U}}_{A}^{>0}$. Since $w^{\prime \prime} x_{i}(h) w^{\prime \prime-1} \in G_{A}$ and $G_{A} \cap \hat{\mathbf{U}}_{A}^{>0}=G_{A}^{>0}$, the result follows.

4.8. In the setup of 2.8 , for any $\mathbf{h}=\left(h_{1}, h_{2}, \ldots, h_{n}\right) \in A^{n}$ we set

$$
\mathbf{x}_{\mathbf{h}}=x_{i_{1}}\left(h_{1}\right)\left(s_{i_{1}}^{\prime \prime} x_{i_{2}}\left(h_{2}\right) s_{i_{1}}^{\prime \prime}-1\right) \ldots\left(s_{i_{1}}^{\prime \prime} s_{i_{2}}^{\prime \prime} \ldots s_{i_{n-1}}^{\prime \prime} x_{i_{n}}\left(h_{n}\right) s_{i_{n-1}}^{\prime \prime}{ }^{-1} \ldots s_{i_{2}}^{\prime \prime-1} s_{i_{1}}^{\prime \prime-1}\right) .
$$

From 4.7(a) we see that $\mathbf{x}_{\mathbf{h}} \in G_{A}^{>0}$. We show:

(a) The map $A^{n} \rightarrow G_{A}^{>0}, \mathbf{h} \mapsto \mathbf{x}_{\mathbf{h}}$ is a bijection.

For $\mathbf{h}$ as above we have (using $2.3(\mathrm{c})$ ):

$$
\begin{aligned}
& \mathbf{x}_{\mathbf{h}}=\sum_{\substack{c_{1}, c_{2}, \ldots, c_{n} \in \mathbf{N}, \lambda_{1}, \lambda_{2}, \ldots, \lambda_{n} \in X \\
c_{1}}} h_{1}^{c_{1}} h_{2}^{c_{2}} \ldots h_{n}^{c_{n}} \\
& \times \theta_{i_{1}}^{\left(c_{1}\right)+} 1_{\lambda_{1}} T_{i_{1}, 1}^{\prime \prime}\left(\theta_{i_{2}}^{\left(c_{2}\right)+} 1_{\lambda_{2}}\right) \ldots T_{i_{1}, 1}^{\prime \prime} T_{i_{2}, 1}^{\prime \prime} \ldots T_{i_{n-1}, 1}^{\prime \prime}\left(\theta_{i_{n}}^{\left(c_{n}\right)+} 1_{\lambda_{n}}\right) \\
& =\sum_{\mathbf{c} \in \mathbf{N}^{n}, \lambda \in X} h_{1}^{c_{1}} h_{2}^{c_{2}} \ldots h_{n}^{c_{n}} x_{\mathbf{c}} 1_{\lambda} .
\end{aligned}
$$

Hence for $\mathbf{c} \in \mathbf{N}^{n}$ we have

$$
\mathbf{x}_{\mathbf{h}}\left(\xi_{\mathbf{c}}\right)=h_{1}^{c_{1}} h_{2}^{c_{2}} \ldots h_{n}^{c_{n}} .
$$

Now the $A$-algebra homomorphisms $A\left[t_{1}, t_{2}, \ldots, t_{n}\right] \rightarrow A$ (preserving 1 ) are clearly in bijection with the points of $A^{n}$; to $\left(h_{1}, h_{2}, \ldots, h_{n}\right) \in A^{n}$ corresponds the algebra homomorphism which takes $t_{r}$ to $h_{r}$ for $r \in[1, n]$. Composing with $\kappa$ in 3.13 , we obtain a bijection between the set of $A$-algebra homomorphisms $\mathbf{o}_{A} \rightarrow A$ (preserving 1) and the set $A^{n}$. From $4.8(\mathrm{~b})$ we see that under this bijection $\mathbf{x}_{\mathbf{h}} \in G_{A}^{>0}$ corresponds to $\mathbf{h} \in A^{n}$. This proves $4.8(\mathrm{a})$. 
4.9. For any $\lambda \in X$ we define a homomorphism $\chi_{\lambda}: T_{A} \rightarrow A^{\circ}$ by $d \otimes y \mapsto d^{\langle y, \lambda\rangle}$ for $d \in A^{\circ}, y \in Y$ or equivalently by $\sum_{\lambda \in X} n_{\lambda} \lambda \mapsto n_{\lambda}$. For $t \in T_{A}, h \in A, i \in I$, we show:

$$
t x_{i}(h) t^{-1}=x_{i}\left(\chi_{i^{\prime}}(t) h\right) .
$$

Writing $t=\sum_{\lambda \in X} n_{\lambda} \lambda$, we see that the left hand side of $4.9(\mathrm{a})$ is

$$
\begin{aligned}
\sum_{c \in \mathbf{N}, \lambda, \lambda^{\prime} \in X} n_{\lambda}^{-1} n_{\lambda^{\prime}} h^{c} 1_{\lambda^{\prime}} \theta_{i}^{(c)+} 1_{\lambda} & =\sum_{c \in \mathbf{N}, \lambda, \lambda^{\prime} \in X} n_{\lambda}^{-1} n_{\lambda^{\prime}} h^{c} \theta_{i}^{(c)+} 1_{\lambda^{\prime}-c i^{\prime}} 1_{\lambda} \\
= & \sum_{c \in \mathbf{N}, \lambda \in X} n_{\lambda}^{-1} n_{\lambda+c i^{\prime}} h^{t} \theta_{i}^{(c)+} 1_{\lambda}=\sum_{c \in \mathbf{N}, \lambda \in X} n_{i^{\prime}}^{c} h^{c} \theta_{i}^{(c)+} 1_{\lambda}=x_{i}\left(n_{i^{\prime}} h\right)=x_{i}\left(\chi_{i^{\prime}}(t) h\right) .
\end{aligned}
$$

This proves $4.9(\mathrm{a})$.

In the setup of 2.8 , let $k \in[1, n]$. Define a homomorphism $f_{k}: A \rightarrow G_{A}^{>0}$ by $h \mapsto s_{i_{1}}^{\prime \prime} s_{i_{2}}^{\prime \prime} \ldots s_{i_{k-1}}^{\prime \prime} x_{i_{k}}(h) s_{i_{k-1}}^{\prime \prime}{ }^{-1} \ldots s_{i_{2}}^{\prime \prime-1} s_{i_{1}}^{\prime \prime-1}$. From 3.13 we see that $f_{k}$ is an isomorphism of $A$ onto its image $f_{k}(A)$. We set $\lambda_{k}=s_{i_{1}} s_{i_{2}} \ldots s_{i_{k-1}}\left(i_{k}^{\prime}\right) \in X$. For $t \in T_{A}, h \in A$ we show:

$$
t f_{k}(h) t^{-1}=f_{k}\left(\chi_{\lambda_{k}}(t) h\right) .
$$

Let $t^{\prime}=s_{i_{k-1}} \ldots s_{i_{2}} s_{i_{1}}(t) \in T_{A}$. Using 4.3(a), we see that 4.9(b) is equivalent to $t^{\prime} x_{i_{k}}(h) t^{\prime-1}=x_{i_{k}}\left(\chi_{\lambda_{k}}(t) h\right)$, that is, using 4.9(a), to $x_{i_{k}}\left(\chi_{i_{k}^{\prime}}\left(t^{\prime}\right) h\right)=x_{i_{k}}\left(\chi_{\lambda_{k}}(t) h\right)$. It is enough to show that $\chi_{i_{k}^{\prime}}\left(t^{\prime}\right)=\chi_{\lambda_{k}}(t)$. This is immediate from the definitions.

We show:

(c) Let $w \in W, i \in I$. There exists $u \in A^{\circ}$ such that for any $h \in A$ we have $\omega\left(w^{\prime \prime} x_{i}(h) w^{\prime \prime-1}\right)=w^{\prime \prime} y_{i}(u h) w^{\prime \prime-1}$.

Writing $w=s_{j_{1}} s_{j_{2}} \ldots s_{j_{r}}$ with $j_{1}, j_{2}, \ldots, j_{r}$ in $I$ and $r=l(w)$, we have

$$
w^{\prime \prime}=s_{j_{1}}^{\prime} t_{j_{1}}(-1) s_{j_{2}}^{\prime} t_{j_{2}}(-1) \ldots s_{j_{r}}^{\prime} t_{j_{r}}(-1)=w^{\prime} t
$$

for some $t \in T_{A}$. Hence

$$
w^{\prime \prime} x_{i}(h) w^{\prime \prime-1}=w^{\prime} t x_{i}(h) t^{-1} w^{\prime-1}=w^{\prime} x_{i}(u h) w^{-1}
$$

where $u=\chi_{i^{\prime}}(t) \in A^{\circ}$; see 4.9(a). Using $\omega\left(w^{\prime \prime}\right)=w^{\prime}, \omega\left(y_{i}(u h)\right)=x_{i}(u h)$, we obtain $w^{\prime \prime} x_{i}(h) w^{\prime \prime-1}=\omega\left(w^{\prime \prime} y_{i}(u h) w^{\prime \prime-1}\right)$ and $4.9(\mathrm{c})$ is proved.

4.10. We now assume that $A$ is an algebraically closed field. Since, by $3.15, \mathbf{O}_{A}$ is a finitely generated $A$-algebra which is an integral domain, we see that $G_{A}$ is a connected algebraic group over $A$ with coordinate ring $\mathbf{O}_{A}$. Since, by $3.13, \mathbf{o}_{A}$ is a finitely generated $A$-algebra which is an integral domain, we see that $\mathfrak{G}_{A}$ (and hence $G_{A}^{>0}$ and $G_{A}^{<0}$ ) are connected algebraic groups over $A$ with coordinate ring $\mathbf{o}_{A}$. Since the inclusions of $G_{A}^{>0}$ and $G_{A}^{<0}$ into $G_{A}$ correspond to surjective algebra homomorphisms $\mathbf{O}_{A} \rightarrow \mathbf{o}_{A}$, we see that $G_{A}^{>0}$ and $G_{A}^{<0}$ are closed subgroups of $G_{A}$. Also $T_{A}$ is clearly a subtorus of $G_{A}$.

Theorem 4.11. In the setup of 4.10, $G_{A}$ is a connected reductive group over $A$ with associated root datum the same as the one given in 1.1.

From 3.13 we see that as an affine variety $G_{A}^{>0}$ is isomorphic to the affine space $A^{n}$. It follows that $G_{A}^{>0}$ is a connected unipotent group. Similarly $G_{A}^{<0}$ is a connected unipotent group.

The $T_{A}$-action (conjugation) on the unipotent group $G_{A}^{>0}$ satisfies the hypotheses of [B1, 14.4, IV]: the induced $T_{A}$-action on Lie $G^{>0}$ is through one dimensional weight spaces corresponding to weights given by the distinct non-zero elements 
$\lambda_{k}(k \in[1, n])$ of $X$ (see 4.9) and $\lambda_{k}, \lambda_{k^{\prime}}$ are linearly independent in $X$ for $k \neq k^{\prime}$. (This is a well known property of the $W$-action on $X$.) Using loc. cit, we see that any non-trivial closed subgroup of $G_{A}^{>0}$ which is stable under conjugation by $T$ contains the subgroup $f_{k}(A)$ (see 4.9) for some $k \in[1, n]$; moreover, the centralizer of $T_{A}$ in $G_{A}^{>0}$ is $\{1\}$.

From 3.10 we see that the morphism $G_{A}^{<0} \times T_{A} \times G_{A}^{>0} \rightarrow G_{A}$ given by multiplication in $G_{A}$ has dense image. Since $T_{A}$ normalizes $G_{A}^{<0}$, we see that $G_{A}^{>0} T_{A}$ is a closed subgroup of $G_{A}$. Since $G_{A}^{<0} T_{A} G_{A}^{>0}$ is the orbit of 1 for an action of $\left(G_{A}^{<0} T\right) \times G_{A}^{>0}$ on $G_{A}$, we see that this orbit is locally closed in $G_{A}$. Since it is dense in $G_{A}$, we see that $G_{A}^{<0} T_{A} G_{A}^{>0}$ is open in $G_{A}$.

Since $G_{A}^{<0} T_{A}$ is solvable and connected, it is contained in some Borel subgroup $B$ of $G_{A}$. Assume that $G_{A}^{<0} T_{A}$ is contained properly in $B$. Now $\left(G_{A}^{<0} T_{A} G_{A}^{>0}\right) \cap B$ is open in $B$. If $\xi_{1}, \xi_{2}, \xi_{3}$ are elements of $G_{A}^{>0}, T_{A}, G_{A}^{>0}$ such that $\xi_{1} \xi_{2} \xi_{3} \in B$, then using $\xi_{1} \xi_{2} \in B$, we see that $\xi_{3} \in B$. Thus $\left(G_{A}^{<0} T_{A} G_{A}^{>0}\right) \cap B=\left(G_{A}^{<0} T_{A}\right)\left(G_{A}^{>0} \cap B\right)$. If $G_{A}^{>0} \cap B=\{1\}$, it would follow that $\left(G_{A}^{>0} T_{A} G_{A}^{>0}\right) \cap B=G_{A}^{<0} T_{A}$ which is a proper closed subset of $B$; this contradicts the fact that $\left(G_{A}^{>0} T_{A} G_{A}^{>0}\right) \cap B$ is open (non-empty) in $B$. Thus we have $G_{A}^{>0} \cap B \neq\{1\}$. Since $G_{A}^{>0} \cap B$ is a unipotent subgroup of $B$, it is equal to $G_{A}^{>0} \cap B_{u}$ where $B_{u}$ is the unipotent radical of $B$. Since $G_{A}^{>0} \cap B_{u}$ is a non-trivial closed subgroup of $G_{A}^{>0}$, stable under conjugation by $T_{A}$, we see that we have $f_{k}(A) \subset G_{A}^{>0} \cap B_{u}$ for some $k \in[1, n]$. Since $f_{k}(A) \subset G_{A}^{>0}$, we have $\omega\left(f_{k}(A)\right) \subset G_{A}^{<0}$. Since $G_{A}^{<0}$ is a unipotent subgroup of $B$, we have $G_{A}^{<0} \subset B_{u}$, hence $\omega\left(f_{k}(A)\right) \subset B_{u}$. Thus there exists $w \in W$ and $i \in I$ such that $w^{\prime \prime} x_{i}(A) w^{\prime \prime} \subset$ $B_{u}$ and $\omega\left(w^{\prime \prime} x_{i}(A) w^{\prime \prime}\right) \subset B_{u}$. Hence, using 4.9(c), we have $w^{\prime \prime} y_{i}(A) w^{\prime \prime-1}$. Thus $x_{i}(A)$ and $y_{i}(A)$ are contained in the unipotent group $w^{\prime \prime-1} B_{u} w^{\prime \prime}$. Using 4.6, we deduce that for any $u \in A^{\circ}$ we have $t_{i}(u) \in w^{\prime \prime-1} B_{u} w^{\prime \prime}$. Since $t_{i}(u) \in T_{A}$ is semisimple, it follows that $t_{i}(u)=1$ for all $u \in A-\{0\}$. From the definition of $t_{i}(u)$ we see that if we choose $u \in A-\{0,1\}$ (recall that $A$ is algebraically closed), then $t_{i}(u) \neq 1$. We have a contradiction. We see that $B=G_{A}^{<0} T_{A}$ so that $G_{A}^{<0} T_{A}$ is a Borel subgroup of $G_{A}$. Applying $\omega$, we see that $\omega\left(G_{A}^{<0} T_{A}\right)=G_{A}^{>0} T_{A}$ is a Borel subgroup of $G_{A}$. From $4.2(\mathrm{a})$ we see that $\left(G_{A}^{<0} T_{A}\right) \cap\left(G_{A}^{>0} T_{A}\right)=T_{A}$. Since the unipotent radical of $G_{A}$ is contained in both Borel subgroups $G_{A}^{<0} T_{A}, G_{A}^{>0} T_{A}$, it must also be contained in their intersection, the torus $T_{A}$. Thus, the unipotent radical of $G_{A}$ is $\{1\}$. We see that $G_{A}$ is reductive. Since $T_{A}$ is the intersection of two Borel subgroups of $G_{A}$, it must be a maximal torus of $G_{A}$. From the definitions we see that the weights of the adjoint action of $T_{A}$ on the Lie algebra of $G_{A}$ are exactly $\lambda_{k}(k \in[1, n])$, their negatives (each with multiplicity 1 ) and the 0 weight with multiplicity $\operatorname{dim} T_{A}$. It follows that the root datum associated to $G_{A}$ is exactly the root datum given in 1.1. The theorem is proved.

\section{From ENVELOPing ALGEBRAS TO MODIFIED ENVELOPING ALGEBRAS}

5.1. In this subsection we recall some definitions from [Ko].

Let $\mathbf{U}_{\mathbf{Q}}$ be the $\mathbf{Q}$-algebra with 1 generated by the symbols $x^{+}, x^{-}$with $x \in \mathbf{f}_{\mathbf{Q}}$ and $\underline{y}$ with $y \in Y$; these symbols are subject to the following relations:

$\mathbf{f}_{\mathbf{Q}} \rightarrow \mathbf{U}_{\mathbf{Q}}, x \mapsto x^{ \pm}$is a $\mathbf{Q}$-algebra homomorphism preserving 1 ;

$Y \rightarrow \mathbf{U}_{\mathbf{Q}}, y \mapsto \underline{y}$ is $\mathbf{Z}$-linear;

$\underline{y y^{\prime}}=\underline{y}^{\prime} \underline{y}$ for $y, y^{\prime} \in Y$; 
$\underline{y}_{i}^{ \pm}=\theta_{i}^{ \pm}(\underline{y} \pm\langle y, i\rangle)$ for $y \in Y, i \in I$

$\bar{\theta}_{i}^{+} \theta_{j}^{-}-\theta_{j}^{-} \bar{\theta}_{i}^{+}=\delta_{i, j} \underline{i}$ for $i, j \in I$.

Thus $\mathbf{U}_{\mathbf{Q}}$ is the universal enveloping algebra attached to our root datum. There is a unique algebra homomorphism $\Delta: \mathbf{U}_{\mathbf{Q}} \rightarrow \mathbf{U}_{\mathbf{Q}} \otimes_{\mathbf{Q}} \mathbf{U}_{\mathbf{Q}}$ such that $\Delta(\underline{y})=$ $\underline{y} \otimes 1+1 \otimes \underline{y}$ for $y \in Y, \Delta\left(\theta_{i}^{ \pm}\right)=\theta_{i}^{ \pm} \otimes 1+1 \otimes \theta_{i}^{ \pm}$for $i \in I$. There is a unique algebra homomorphism $S$ from $\mathbf{U}_{\mathbf{Q}}$ to $\mathbf{U}_{\mathbf{Q}}$ with the opposed multiplication such that $S(\underline{y})=-\underline{y}$ for $y \in Y, S\left(\theta_{i}^{ \pm}\right)=-\theta_{i}^{ \pm}$for $i \in I$. There is a unique algebra homomorphism $\epsilon: \mathbf{U}_{\mathbf{Q}} \rightarrow \mathbf{Q}$ preserving 1 such that $\epsilon(y)=0$ for $y \in Y, \epsilon\left(\theta_{i}^{ \pm}\right)=0$ for $i \in I$. Then $\left(\mathbf{U}_{\mathbf{Q}}, \Delta, S\right)$ is a Hopf algebra over $\mathbf{Q}$ with 1 and with counit $\epsilon$.

Let $\mathfrak{C}_{\mathbf{Q}}^{\prime}$ be the category whose objects are unital $\mathbf{U}_{\mathbf{Q}}$-modules $M$ such that $M=\bigoplus_{\lambda \in X} M^{\lambda}$ (as a vector space) where for any $\lambda \in X$ we have $M^{\lambda}=\{z \in$ $M ; \underline{y} z=\langle y, \lambda\rangle z$ for any $y \in Y\}$.

For any $M \in \mathfrak{C}_{\mathbf{Q}}^{\prime}$ let $C_{M}^{\prime}$ be the $\mathbf{Q}$-subspace of $\mathbf{U}_{\mathbf{Q}}^{\diamond}$ spaned by the functions $u \mapsto$ $z^{\prime}(u z)$ for various $z \in M, z^{\prime} \in M^{\diamond}$. Let $\mathbf{O}_{\mathbf{Q}}^{\prime}=\sum_{M \in \mathfrak{C}_{\mathbf{Q}}^{\prime}} C_{M}^{\prime}$, a $\mathbf{Q}$-subspace of $\mathbf{U}_{\mathbf{Q}}^{\diamond}$. Now $\mathbf{O}_{\mathbf{Q}}^{\prime}$ is a Hopf agebra over $\mathbf{Q}$ in which the multiplication is the "transpose" of $\Delta: \mathbf{U}_{\mathbf{Q}} \rightarrow \mathbf{U}_{\mathbf{Q}} \otimes_{\mathbf{Q}} \mathbf{U}_{\mathbf{Q}}$, the comultiplication is the "transpose" of the multiplication $\mathbf{U}_{\mathbf{Q}} \otimes_{\mathbf{Q}} \mathbf{U}_{\mathbf{Q}} \rightarrow \mathbf{U}_{\mathbf{Q}}$, the antipode is the "transpose" of $S: \mathbf{U}_{\mathbf{Q}} \rightarrow \mathbf{U}_{\mathbf{Q}}$, the unit is the counit of $\mathbf{U}_{\mathbf{Q}}$ and the counit is given by $f \mapsto f(1)$.

Let $\mathbf{U}_{\mathbf{Z}}$ be the subring of $\mathbf{U}_{\mathbf{Q}}$ generated by the elements

$$
\left(\frac{y}{k}\right)=\underline{y}(\underline{y}-1) \ldots(\underline{y}-k+1) / k !
$$

for $y \in Y, k \in \mathbf{N}$ and $\theta_{i}^{(m)+}, \theta_{i}^{(m)-}$ for $i \in I, m \in \mathbf{N}$. This is a $\mathbf{Z}$-lattice in $\mathbf{U}_{\mathbf{Q}}$, called the Kostant $\mathbf{Z}$-form of $\mathbf{U}_{\mathbf{Q}}$. Let $\mathbf{O}_{\mathbf{Z}}^{\prime}=\left\{f \in \mathbf{O}_{\mathbf{Q}}^{\prime} ; f\left(\mathbf{U}_{\mathbf{Z}}\right) \subset \mathbf{Z}\right\}$. This is a $\mathbf{Z}$-subalgebra with 1 of $\mathbf{O}_{\mathbf{Q}}^{\prime}$; it inherits from $\mathbf{O}_{\mathbf{Q}}^{\prime}$ a comultiplication, an antipode and a counit, which make it into a Hopf algebra over $\mathbf{Z}$ (here we use the fact that $\mathbf{O}_{\mathbf{Z}}^{\prime}$ is a torsion free $\mathbf{Z}$-module).

If $v=1$ in $A$, we set $\mathbf{O}_{A}^{\prime}=A \otimes \mathbf{O}_{\mathbf{Z}}^{\prime}$; this is naturally a Hopf algebra over $A$. The following results show that our results about $\mathbf{O}_{A}$ apply also to $\mathbf{O}_{A}^{\prime}$.

Proposition 5.2. If $v=1$ in $A$, we have canonically $\mathbf{O}_{A}^{\prime}=\mathbf{O}_{A}$ as Hopf algebras over A.

The proof is given in 5.9 .

5.3. Let $\mathbf{P}$ be the $\mathbf{Q}$-algebra of functions $X \rightarrow \mathbf{Q}$ generated by the functions $\underline{y}: \lambda \mapsto\langle y, \lambda\rangle$ for various $y \in Y$. We regard $\mathbf{P}$ as a $\mathbf{Q}$-subalgebra with 1 of $\hat{\mathbf{U}}_{\mathbf{Q}}^{0}$ by $\bar{\phi} \mapsto \sum_{\lambda \in X} \phi(\lambda) 1_{\lambda}$. For $y \in Y, h \in \mathbf{Z}$ and $k \in \mathbf{N}$ we set

$$
\left(\begin{array}{c}
\underline{y}+h \\
k
\end{array}\right)=(\underline{y}+h)(\underline{y}+h-1) \ldots(\underline{y}+h-k+1) / k ! \in \mathbf{P} .
$$

Let $\mathbf{P}_{\mathbf{Z}}$ be the subring of $\mathbf{P}$ generated by the elements $\left(\frac{y}{k}\right)$ for various $y \in Y, k \in \mathbf{N}$. Note that

(a) $\mathbf{P}_{\mathbf{Z}} \subset \hat{\mathbf{U}}_{\mathbf{Z}}^{0}$

(b) $\left(\underline{y}_{k}^{+h}\right) \in \mathbf{P}_{\mathbf{Z}}$ for any $y \in Y, h \in \mathbf{Z}, k \in \mathbf{N}$.

Let $\left\{y_{1}, y_{2}, \ldots, y_{r}\right\}$ be a basis of the $\mathbf{Z}$-module $Y$. The following result is well known. 
(c) The elements $\phi_{\mathbf{k}}=\left(\frac{y_{1}}{k_{1}}\right)\left(\frac{y_{2}}{k_{2}}\right) \ldots\left(\frac{y_{r}}{k_{r}}\right)$ (with $\left.\mathbf{k}=\left(k_{1}, k_{2}, \ldots, k_{r}\right) \in \mathbf{N}^{r}\right)$ form a $\mathbf{Z}$-basis of the $\mathbf{Z}$-module $\mathbf{P}_{\mathbf{Z}}$ and a $\mathbf{Q}$-basis of the $\mathbf{Q}$-vector space $\mathbf{P}$.

We show:

(d) Let $N \geq 1$. Let $X_{N}=\left\{\lambda \in X ;-N \leq\left\langle y_{j}, \lambda\right\rangle \leq N\right.$ for $\left.j=1,2, \ldots, r\right\}$. Let $\mathcal{M}_{N}$ be the set of all elements $\sum_{\lambda^{\prime} \in X} n_{\lambda^{\prime}} 1_{\lambda^{\prime}} \in \hat{\mathbf{U}}_{\mathbf{Z}}^{0}$ such that $n_{\lambda^{\prime}}=0$ for $\lambda^{\prime} \in X_{N}$. Let $\lambda \in X$. Then $1_{\lambda} \in \mathbf{P}_{\mathbf{z}}+\mathcal{M}_{N}$.

If $\lambda \notin X_{N}$, then $1_{\lambda} \in \mathcal{M}_{N}$ and the result holds. Now assume that $\lambda \in X_{N}$. We have

(e) $\prod_{j \in[1, r]}\left(\begin{array}{c}y_{j}+2 N-\left\langle y_{j}, \lambda\right\rangle \\ 2 N\end{array}\right)=\sum_{\substack{\lambda^{\prime} \in X ;\left\langle y_{j}, \lambda^{\prime}\right\rangle \in\left(\infty,-\left\langle y_{j}, \lambda\right\rangle-2 N-1\right] \cup\left[\left\langle y_{j}, \lambda\right\rangle, \infty\right) \\ \text { for } j \in[1, r]}} n_{\lambda^{\prime}} 1_{\lambda^{\prime}}$

where $n_{\lambda^{\prime}} \in \mathbf{Z}$ are such that $n_{\lambda}=1$. For $\lambda^{\prime}$ in the last sum such that $\left\langle y_{j}, \lambda^{\prime}\right\rangle \in$ $\left(\infty,-\left\langle y_{j}, \lambda\right\rangle-2 N-1\right]$ for some $j$ we have $\left\langle y_{j}, \lambda^{\prime}\right\rangle \leq-N-1$ (since $\left.-N \leq\left\langle y_{j}, \lambda\right\rangle\right)$, hence $\lambda^{\prime} \notin X_{N}$; the sum over all such $\lambda^{\prime}$ is in $\mathcal{M}_{N}$. On the other hand, the left hand side of 5.3(e) is in $\mathbf{P}_{\mathbf{Z}}$. Thus 5.3(e) implies

$$
\sum_{\lambda^{\prime} \in X_{N} ;\left\langle y_{j}, \lambda^{\prime}\right\rangle \geq\left\langle y_{j}, \lambda\right\rangle \text { for } j \in[1, r]} n_{\lambda^{\prime}} 1_{\lambda^{\prime}} \in \mathbf{P}_{\mathbf{Z}}+\mathcal{M}_{N}
$$

where $n_{\lambda^{\prime}} \in \mathbf{Z}$ are such that $n_{\lambda}=1$. For any $\lambda^{\prime} \in X_{N}$ we set $q_{\lambda^{\prime}}=\sum_{j \in[1, r]}\left\langle y_{j}, \lambda^{\prime}\right\rangle$ so that $-N r \leq q_{\lambda^{\prime}} \leq N r$. We have

$$
1_{\lambda}+\sum_{\left.\lambda^{\prime} \in X_{N} ;\left\langle y_{j}, \lambda^{\prime}\right\rangle \geq\left\langle y_{j}, \lambda\right\rangle \text { for } j \in[1, r], q_{\lambda^{\prime}}\right\rangle q_{\lambda}} n_{\lambda^{\prime}} 1_{\lambda^{\prime}} \in \mathbf{P}_{\mathbf{Z}}+\mathcal{M}_{N} .
$$

This shows by descending induction on $q_{\lambda}$ (starting with $q_{\lambda}=N r$ ) that $1_{\lambda} \in$ $\mathbf{P}_{\mathbf{Z}}+\mathcal{M}_{N}$ for any $\lambda \in X_{N}$. This proves 5.3(d).

5.4. There is a unique algebra homomorphism $\mathbf{U}_{\mathbf{Q}} \rightarrow \hat{\mathbf{U}}_{\mathbf{Q}}$ preserving 1 such that $\underline{y} \mapsto \sum_{\lambda \in X}\langle y, \lambda\rangle 1_{\lambda}$ for any $y \in Y, \theta_{i}^{ \pm} \mapsto \theta_{i}^{ \pm}$(as in 2.7) for any $i \in I$. This homomorphism is injective and we identify $\mathbf{U}_{\mathbf{Q}}$ with a subalgebra of $\hat{\mathbf{U}}_{\mathbf{Q}}$ via this homomorphism. We identify $\mathbf{P}$ with a subalgebra of $\mathbf{U}_{\mathbf{Q}}$ by $\underline{y} \mapsto \underline{y}$. This makes $\mathbf{P}$ a subalgebra of $\hat{\mathbf{U}}_{\mathbf{Q}}^{0}$ (in the same way as in 5.3). Note that:

(a) The elements $b^{+} \phi_{\mathbf{k}} b^{\prime-}$ with $b, b^{\prime} \in \mathbf{B}$ and $\mathbf{k} \in \mathbf{N}^{r}$ form a $\mathbf{Z}$-basis of $\mathbf{U}_{\mathbf{Z}}$ and a $\mathbf{Q}$-basis of $\mathbf{U}_{\mathbf{Q}}$.

We show that

(b) $\mathbf{U}_{\mathbf{Z}} \subset \hat{\mathbf{U}}_{\mathbf{Z}}$.

By 5.4(a) it is enough to show that for $b \in \mathbf{B}$ we have $b^{+} \in \hat{\mathbf{U}}_{\mathbf{Z}}$ (this is clear), $b^{-} \in \hat{\mathbf{U}}_{\mathbf{Z}}$ (this is clear) and that for $\mathbf{k} \in \mathbf{N}$ we have $\phi_{\mathbf{k}} \in \hat{\mathbf{U}}_{\mathbf{Z}}^{0}$ (this follows from $5.3(\mathrm{a}))$.

The category $\mathfrak{C}_{\mathbf{Q}}$ (see 1.6) is equivalent to the category $\mathfrak{C}_{\mathbf{Q}}^{\prime}$ : if $M \in \mathfrak{C}_{\mathbf{Q}}$, then $M$ can be regarded as a $\hat{\mathbf{U}}_{\mathbf{Q}}$-module as in 1.12 and then as a $\mathbf{U}_{\mathbf{Q}}$-module via the

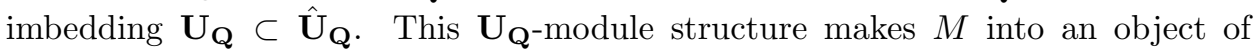
$\mathfrak{C}_{\mathbf{Q}}^{\prime}$. This gives a functor $\mathfrak{C}_{\mathbf{Q}} \rightarrow \mathfrak{C}_{\mathbf{Q}}^{\prime}$ which is easily seen to be an equivalence of categories. It is well known that

(c) Any object in $\mathfrak{C}_{\mathbf{Q}}$ is semisimple and the simple objects of $\mathfrak{C}_{\mathbf{Q}}$ are exactly the objects $\Lambda_{\lambda, \mathbf{Q}}$ for various $\lambda \in X^{+}$.

For any $M \in \mathfrak{C}_{\mathbf{Q}}$ let $C_{M}$ be the $\mathbf{Q}$-subspace of $\dot{\mathbf{U}}_{\mathbf{Q}}^{\diamond}$ spanned by the functions $u \mapsto z^{\prime}(u z)$ for various $z \in M, z^{\prime} \in M^{\diamond}$. 
Lemma 5.5. Let $f \in \dot{\mathbf{U}}_{\mathbf{Q}}^{\diamond}$. The following three properties are equivalent:

(i) $f \in \mathbf{O}_{\mathbf{Q}}$;

(ii) $f \in \sum_{M \in \mathfrak{C}_{\mathbf{Q}}} C_{M}$.

(iii) $f \in \sum_{\lambda \in X^{+}} C_{\Lambda_{\lambda, \mathbf{Q}}}$.

Assume that (i) holds. To show (ii), we can assume that $f=a^{*}$ where $a \in \dot{\mathbf{B}}_{\lambda, \lambda^{\prime}}$ for some $\lambda, \lambda^{\prime}$ in $X^{+}$. Let $M={ }^{\omega} \Lambda_{\lambda, \mathbf{Q}} \otimes_{\mathbf{Q}} \Lambda_{\lambda^{\prime}, \mathbf{Q}}$. Let $z=\xi_{\lambda} \otimes \eta_{\lambda^{\prime}} \in M$. Define $z^{\prime} \in M^{\diamond}$ by $z^{\prime}\left(a^{\prime} z\right)=\delta_{a, a^{\prime}}$ for any $a^{\prime} \in \dot{\mathbf{B}}_{\lambda, \lambda^{\prime}}$. For $a^{\prime \prime} \in \mathbf{\mathbf { B }}$ we have $z^{\prime}\left(a^{\prime \prime} z\right)=a^{*}\left(a^{\prime \prime}\right)$ (if $a^{\prime \prime} \notin \dot{\mathbf{B}}_{\lambda, \lambda^{\prime}}$, both sides are 0 since $a^{\prime \prime} z=0$; if $a^{\prime \prime} \in \dot{\mathbf{B}}_{\lambda, \lambda^{\prime}}$, both sides are $\delta_{a, a^{\prime \prime}}$ ). Hence $z^{\prime}(u z)=a^{*}(u)$ for all $u \in \dot{\mathbf{U}}_{\mathbf{Q}}$. Hence $a^{*} \in C_{M}$, as required.

Assume that (iii) holds. To show (i), we may assume that there exist $z \in M$, $z^{\prime} \in M^{\diamond}$ with $M=\Lambda_{\lambda, \mathbf{Q}}, \lambda \in X^{+}$such that $f(u)=z^{\prime}(u z)$ for all $u \in \dot{\mathbf{U}}_{\mathbf{Q}}$. Recall that, if $b \in \dot{\mathbf{B}}-\bigcup_{\lambda^{\prime} ; \lambda \geq \lambda^{\prime}} \dot{\mathbf{B}}\left[\lambda^{\prime}\right]$, then $b$ acts as 0 on $M$ and hence $f(b)=0$. Let $f_{1}=f-\sum_{b \in \cup_{\lambda^{\prime} ; \lambda \geq \lambda^{\prime}} \dot{\mathbf{B}}\left[\lambda^{\prime}\right]} f(b) b^{*} \in \dot{\mathbf{U}}_{\mathbf{Q}}^{\diamond}$. Note that $f_{1}(b)=0$ if $b \in \bigcup_{\lambda^{\prime} ; \lambda \geq \lambda^{\prime}} \dot{\mathbf{B}}\left[\lambda^{\prime}\right]$. If $b^{\prime} \notin \bigcup_{\lambda^{\prime} ; \lambda \geq \lambda^{\prime}} \dot{\mathbf{B}}\left[\lambda^{\prime}\right]$, then both $f$ and $\sum_{b \in \bigcup_{\lambda^{\prime} ; \lambda \geq \lambda^{\prime}} \dot{\mathbf{B}}\left[\lambda^{\prime}\right]} f(b) b^{*}$ vanish on $b^{\prime}$. Hence $f_{1}\left(b^{\prime}\right)=0$. We see that $f_{1}=0$ and $f=\sum_{b \in \cup_{\lambda^{\prime} ; \lambda \geq \lambda^{\prime}} \dot{\mathbf{B}}\left[\lambda^{\prime}\right]} f(b) b^{*}$. Thus $f \in \mathbf{O}_{\mathbf{Q}}$, as required.

Assume that (ii) holds. Then (iii) holds since $C_{M^{\prime} \oplus M^{\prime \prime}}=C_{M^{\prime}}+C_{M^{\prime \prime}}$ for any $M^{\prime}, M^{\prime \prime}$ in $\mathfrak{C}_{\mathrm{Q}}$ and any $M \in \mathfrak{C}_{\mathrm{Q}}$ is a direct sum of finitely many objects of the form $\Lambda_{\lambda, \mathbf{Q}}, \lambda \in X^{+}$. The lemma is proved.

5.6. As in the proof of Lemma 5.5 we see that for $f \in \mathbf{U}_{\mathbf{Q}}^{\diamond}$ the following two properties are equivalent:

(i) $f \in \sum_{M \in \mathfrak{C}_{Q}} C_{M}^{\prime}$.

(ii) $f \in \sum_{\lambda \in X^{+}} C_{\Lambda_{\lambda, Q}}^{\prime}$.

(Recall that $\mathfrak{C}_{\mathbf{Q}}=\mathfrak{C}_{\mathbf{Q}}^{\prime}$.)

5.7. Let $\mathcal{O}=\bigoplus_{\lambda \in X^{+}} \Lambda_{\lambda, \mathbf{Q}} \otimes_{\mathbf{Q}} \Lambda_{\lambda, \mathbf{Q}}^{\diamond}$, a $\mathbf{Q}$-vector space. We show:

(a) The linear map $\alpha: \mathcal{O} \rightarrow \dot{\mathbf{U}}_{\mathbf{Q}}^{\diamond}$ whose restriction to $\Lambda_{\lambda, \mathbf{Q}} \otimes_{\mathbf{Q}} \Lambda_{\lambda, \mathbf{Q}}^{\diamond}$ is $z \otimes z^{\prime} \mapsto$ $\left[u \mapsto z^{\prime}(u z)\right]$ is injective.

For any finite subset $F$ of $X^{+}$let $\mathcal{O}_{F}=\bigoplus_{\lambda \in F} \Lambda_{\lambda, \mathbf{Q}} \otimes_{\mathbf{Q}} \Lambda_{\lambda, \mathbf{Q}}^{\diamond}$. It is enough to verify that for any $F$ as above, the linear map $\mathcal{O}_{F} \rightarrow \dot{\mathbf{U}}^{\diamond}$ (restriction of $\mathcal{O} \rightarrow \dot{\mathbf{U}}^{\diamond}$ in $5.7(\mathrm{a}))$ is injective.

Let $\left(\xi_{\lambda}\right)_{\lambda \in F} \in \operatorname{ker}\left(\mathcal{O}_{F} \rightarrow \dot{\mathbf{U}}^{\diamond}\right)$. For $\lambda \in F$ we have $\xi_{\lambda}=\sum_{j} z_{j}^{\lambda} \otimes z_{j}^{\prime \lambda}$ with $z_{j}^{\lambda} \in$ $\Lambda_{\lambda, \mathbf{Q}}, z_{j}^{\prime \lambda} \in \Lambda_{\lambda, \mathbf{Q}}^{\diamond}$. For any $u \in \dot{\mathbf{U}}_{\mathbf{Q}}$ we have $\sum_{\lambda \in F} \sum_{j} z_{j}^{\prime \lambda}\left(u z_{j}^{\lambda}\right)=0$. Using 5.4(c), we see that for any collection $\left(e_{\lambda}\right)_{\lambda \in F}, e_{\lambda} \in \operatorname{End}\left(\Lambda_{\lambda, \mathbf{Q}}\right)$, we can find $u \in \dot{\mathbf{U}}_{\mathbf{Q}}$ such that $u$ acts on $\Lambda_{\lambda, \mathbf{Q}}$ as $e_{\lambda}$. Hence we have $\sum_{\lambda \in F} \sum_{j} z_{j}^{\prime \lambda}\left(e_{\lambda} z_{j}^{\lambda}\right)=0$ for any $\left(e_{\lambda}\right)_{\lambda \in F}$ as above. Hence for any $\lambda \in F$ we have $\sum_{j} z_{j}^{\prime \lambda}\left(e z_{j}^{\lambda}\right)=0$ for any $e \in \operatorname{End}\left(\Lambda_{\lambda, \mathbf{Q}}\right)$. Hence $\sum_{j} z_{j}^{\lambda} \otimes z_{j}^{\prime \lambda}=0$ for any $\lambda \in F$. Thus $\xi_{\lambda}=0$ for any $\lambda \in F$. This proves $5.7(\mathrm{a})$.

An entirely similar proof gives the following result.

(b) The linear map $\alpha^{\prime}: \mathcal{O} \rightarrow \mathbf{U}_{\mathbf{Q}}^{\diamond}$ whose restriction to $\Lambda_{\lambda, \mathbf{Q}} \otimes_{\mathbf{Q}} \Lambda_{\lambda, \mathbf{Q}}^{\diamond}$ is $z \otimes z^{\prime} \mapsto$ $\left[u \mapsto z^{\prime}(u z)\right]$ is injective.

From 5.5, 5.6, we see that the image of $\alpha$ is $\mathbf{O}_{\mathbf{Q}}$ and the image of $\alpha^{\prime}$ is $\mathbf{O}_{\mathbf{Q}}^{\prime}$. Using 5.7(a), (b), we see that $\alpha, \alpha^{\prime}$ define isomorphisms $\mathcal{O} \stackrel{\sim}{\longrightarrow} \mathbf{O}_{\mathbf{Q}}, \mathcal{O} \stackrel{\sim}{\longrightarrow} \mathbf{O}_{\mathbf{Q}}^{\prime}$. 
Now let $\hat{\alpha}: \mathcal{O} \rightarrow \hat{\mathbf{U}}_{\mathbf{Q}}^{\diamond}$ be the linear map whose restriction to $\Lambda_{\lambda, \mathbf{Q}} \otimes_{\mathbf{Q}} \Lambda_{\lambda, \mathbf{Q}}^{\diamond}$ is $z \otimes z^{\prime} \mapsto\left[u \mapsto z^{\prime}(u z)\right]$. Let $\gamma: \hat{\mathbf{U}}_{\mathbf{Q}}^{\diamond} \rightarrow \dot{\mathbf{U}}_{\mathbf{Q}}^{\diamond}, \gamma^{\prime}: \hat{\mathbf{U}}_{\mathbf{Q}}^{\diamond} \rightarrow \mathbf{U}_{\mathbf{Q}}^{\diamond}$ be the linear maps transpose to the imbeddings $\dot{\mathbf{U}}_{\mathbf{Q}} \rightarrow \hat{\mathbf{U}}_{\mathbf{Q}}, \mathbf{U}_{\mathbf{Q}} \rightarrow \hat{\mathbf{U}}_{\mathbf{Q}}$. From the definitions we have $\gamma \hat{\alpha}=\alpha, \gamma^{\prime} \hat{\alpha}=\alpha^{\prime}$. Since $\alpha$ is injective, we see that $\hat{\alpha}$ is injective. Hence $\hat{\alpha}$ defines an isomorphism $\mathcal{O} \rightarrow \hat{\mathbf{O}}_{\mathbf{Q}}$ where $\hat{\mathbf{O}}_{\mathbf{Q}}$ is the image of $\hat{\alpha}$. We see that the image of $\mathbf{O}_{\mathbf{Q}}$ under $\gamma$ is equal to the image of $\mathbf{O}_{\mathbf{Q}}^{\prime}$ under $\gamma^{\prime}$ and both these images are equal to $\hat{\mathbf{O}}_{\mathbf{Q}}$. Now $\gamma, \gamma^{\prime}$ restrict to isomorphisms $\mathbf{O}_{\mathbf{Q}} \stackrel{\sim}{\longrightarrow} \hat{\mathbf{O}}_{\mathbf{Q}}, \mathbf{O}_{\mathbf{Q}}^{\prime} \stackrel{\sim}{\longrightarrow} \hat{\mathbf{O}}_{\mathbf{Q}}$. Thus both $\mathbf{O}_{\mathbf{Q}}, \mathbf{O}_{\mathbf{Q}}^{\prime}$ can be canonically identified with $\hat{\mathbf{O}}_{\mathbf{Q}}$ and hence also with each other. From the definitions we see that this identification of $\mathbf{O}_{\mathbf{Q}}, \mathbf{O}_{\mathbf{Q}}^{\prime}$ is compatible with the Hopf algebra structures.

Lemma 5.8. Let $F$ be a finite subset of $X^{+}$.

(i) Let $u \in \dot{\mathbf{U}}_{\mathbf{Z}}$. There exists $u^{\prime} \in \mathbf{U}_{\mathbf{Z}}$ such that $u, u^{\prime}$ act in the same way on $\Lambda_{\lambda, \mathbf{Q}}$ for any $\lambda \in F$.

(ii) Let $u^{\prime} \in \mathbf{U}_{\mathbf{Z}}$. There exists $u \in \dot{\mathbf{U}}_{\mathbf{Z}}$ such that $u, u^{\prime}$ act in the same way on $\Lambda_{\lambda, \mathbf{Q}}$ for any $\lambda \in F$.

We can find $N \geq 1$ such that $\left\{\lambda \in X ; 1_{\lambda} \Lambda_{\lambda, \mathbf{Q}} \neq 0\right\} \subset X_{N}$ (notation of $5.3(\mathrm{~d})$ ).

To prove Lemma 5.8(i), we may assume that $u=b^{+} 1_{\lambda} b^{\prime-} \in \dot{\mathbf{U}}_{\mathbf{Z}}$ where $b, b^{\prime} \in \mathbf{B}$, $\lambda \in X$. By 5.3(d) we can find $u_{1} \in \mathbf{P}_{\mathbf{Z}}, u_{2} \in \hat{\mathbf{U}}_{\mathbf{Z}}^{0}$ such that $1_{\lambda}=u_{1}+u_{2}$ and $u_{2}$ acts as 0 on $\Lambda_{\lambda, \mathbf{Q}}$ for any $\lambda \in F$. Then $b^{+} u_{2} b^{-} \in \hat{\mathbf{U}}_{\mathbf{Z}}$ acts as 0 on $\Lambda_{\lambda, \mathbf{Q}}$ for any $\lambda \in F$. Hence $u=b^{+} u_{1} b^{-}+b^{+} u_{2} b^{\prime-}$ acts in the same way as $b^{+} u_{1} b^{-}$on $\Lambda_{\lambda, \mathbf{Q}}$ for any $\lambda \in F$. Hence $u^{\prime}=b^{+} u_{1} b^{-} \in \mathbf{U}_{\mathbf{Z}}$ is as required in Lemma 5.8(i).

We prove Lemma 5.8(ii). We set $u=u^{\prime} \sum_{\lambda \in X_{N}} 1_{\lambda} \in \dot{\mathbf{U}}_{\mathbf{Q}}$. Since $u^{\prime} \in \hat{\mathbf{U}}_{\mathbf{Z}}$ (see 5.4(b)), we see that $u \in \hat{\mathbf{U}}_{\mathbf{Z}}$. Thus, $u \in \dot{\mathbf{U}}_{\mathbf{Q}} \cap \hat{\mathbf{U}}_{\mathbf{Z}}=\dot{\mathbf{U}}_{\mathbf{Z}}$. Clearly, $u$ is as required by Lemma 5.8(ii). The lemma is proved.

5.9. We prove Proposition 5.2. We can assume that $A=\mathbf{Z}$. Let $Z$ (resp. $Z^{\prime}$ ) be the set of all $\left(\xi_{\lambda}\right)_{\lambda \in X^{+}} \in \mathcal{O}, \xi_{\lambda}=\sum_{j} z_{j}^{\lambda} \otimes z_{j}^{\prime \lambda}$ with $z_{j}^{\lambda} \in \Lambda_{\lambda, \mathbf{Q}}, z_{j}^{\prime \lambda} \in \Lambda_{\lambda, \mathbf{Q}}^{\diamond}$, such that for any $u \in \dot{\mathbf{U}}_{\mathbf{Z}}$ (resp. any $u \in \mathbf{U}_{\mathbf{Z}}$ ) we have $\sum_{\lambda \in X^{+}} \sum_{j} z_{j}^{\prime \lambda}\left(u z_{j}^{\lambda}\right) \in \mathbf{Z}$.

Under the identification $\mathcal{O}=\mathbf{O}_{\mathbf{Q}}\left(\right.$ resp. $\left.\mathcal{O}=\mathbf{O}_{\mathbf{Q}}^{\prime}\right)$ induced by $\alpha\left(\right.$ resp. $\left.\alpha^{\prime}\right)$ in 5.7, the subset $\mathbf{O}_{\mathbf{Z}}$ (resp. $\mathbf{O}_{\mathbf{Z}}^{\prime}$ ) corresponds to the subset $Z$ (resp. $Z^{\prime}$ ) of $\mathcal{O}$.

Since any element $\left(\xi_{\lambda}\right)_{\lambda \in X^{+}} \in \mathcal{O}$ has only finitely many non-zero components, we see, using Lemma 5.8(i), that $Z^{\prime} \subset Z$ and, using Lemma 5.8(ii), that $Z \subset Z^{\prime}$. Thus $Z=Z^{\prime}$. It follows that under the identification $\mathbf{O}_{\mathbf{Q}}=\mathbf{O}_{\mathbf{Q}}^{\prime}, \mathbf{O}_{\mathbf{Z}}$ corresponds to $\mathbf{O}_{\mathbf{Z}}^{\prime}$. Proposition 5.2 follows.

\section{REFERENCES}

[B1] A. Borel, Linear algebraic groups, W.A. Benjamin, Inc., New York and Amsterdam, 1969. MR0251042 (40:4273)

[B2] A. Borel et al., Seminar on Algebraic Groups and Related Finite Groups, Lecture Notes in Mathematics 131, Springer Verlag, 1970. MR0258838(41:3484)

[C1] C. Chevalley, Sur certains groupes simples, Tohoku Math. J. 7 (1955), 14-66. MR0073602 $(17: 457 \mathrm{c})$

[C2] C. Chevalley, Certains schémas de groupes semi-simples, Sém. Bourbaki 1960/61, Soc. Math. France, 1995. MR1611814

[DG] M. Demazure and A. Grothendieck, Schémas en groupes, Séminaire de Géométrie Algébrique du Bois Marie 1962/64 (SGA3), Lecture Notes in Mathematics 151-153, Springer Verlag, 1970. 
[Jo] A. Joseph, Quantum groups and their primitive ideals, Ergebnisse der Mathematik und ihrer Grenzgebiete, Band 29, Springer Verlag, 1995. MR 1315966 (96d:17015)

[Ko] B. Kostant, Groups over Z, Algebraic Groups and Their Discontinuous Subgroups, Proc. Symp. Pure Math., vol. 8, Amer. Math. Soc., 1966, pp. 90-98. MR0207713 (34:7528)

[L1] G. Lusztig, Introduction to quantum groups, Progress in Math., vol. 110, Birkhäuser, 1993. MR:1227098 (94m:17016)

[L2] G. Lusztig, Quantum groups at $v=\infty$, Functional analysis on the eve of the 21st century, I, Progr. in Math., vol. 131, Birkhäuser, Boston, 1995, pp. 199-221. MR.1373004 (97g:17014)

[So] Y.S. Soibelman, The algebra of functions on a compact quantum group and its irreducible representations, Leningrad Math. J. 2 (1991), 161-178. MR1049910(91i:58053a)

[St] R. Steinberg, Lectures on Chevalley groups, Yale University, 1968. MR0466335 (57:6215)

Department of Mathematics, Massachusetts Institute of Technology, Cambridge, MASSACHUSETTS 02139

E-mail address: gyuri@math.mit.edu 